\title{
Why There is a Field Algebra with a Compact Gauge Group Describing the Superselection Structure in Particle Physics
}

\author{
Sergio Doplicher ${ }^{1 \star}$ and John E. Roberts ${ }^{2}$ \\ 1 Dipartimento di Matematica, Università di Roma "La Sapienza", I-00185 Roma, Italy \\ 2 Fachbereich Physik, Universität Osnabrück, D-4500 Osnabrück, Federal Republic of Germany
}

\begin{abstract}
Given the local observables in the vacuum sector fulfilling a few basic principles of local quantum theory, we show that the superselection structure, intrinsically determined a priori, can always be described by a unique compact global gauge group acting on a field algebra generated by field operators which commute or anticommute at spacelike separations. The field algebra and the gauge group are constructed simultaneously from the local observables. There will be sectors obeying parastatistics, an intrinsic notion derived from the observables, if and only if the gauge group is non-Abelian. Topological charges would manifest themselves in field operators associated with spacelike cones but not localizable in bounded regions of Minkowski space. No assumption on the particle spectrum or even on the covariance of the theory is made. However the notion of superselection sector is tailored to theories without massless particles. When translation or Poincaré covariance of the vacuum sector is assumed, our construction leads to a covariant field algebra describing all covariant sectors.
\end{abstract}

\section{Introduction}

If one starts from local observables which are assumed to be given a priori and to satisfy a few basic principles characteristic of local quantum theory, then the superselection structure of the theory is determined in an intrinsic manner. In this paper, we solve a long-standing problem by showing that this superselection structure can always be described, in a sense that will be made precise, in terms of a compact group of gauge automorphisms of a field net with normal Bose and Fermi commutation relations.

This is a remarkable result for several reasons: first of all we start from local observables which are by their nature gauge invariant so there is no hint of any gauge symmetry. Secondly, although we do have to define carefully what is meant

\footnotetext{
« Research supported by Ministero della Pubblica Istruzione and CNR-GNAFA
} 
by a superselection sector, we make no postulates on the structure of these sectors. Thirdly, we assume neither the existence nor the commutation properties of any unobservable field quantities; all that is assumed is that, as a reflection of Einstein causality, local observables commute at spacelike separations.

These results stem above all from the existence of an intrinsically defined statistics (permutation symmetry) of superselection sectors, established in earlier work [1]. This rules out a gauge symmetry described by a more general object such as a (compact) quantum group or paragroup. It also allows us to introduce fields which obey normal (i.e. Bose-Fermi) commutation relations. However parastatistics is not ruled out by our results: on the contrary, they necessarily arise whenever our gauge group is non-Abelian. A superselection sector with parastatistics of order $d$ corresponds to a multiplet ${ }^{1}$ of fields transforming under a $d$-dimensional irreducible representation of the gauge group.

From a mathematical point of view, the problem we have solved is one in the duality theory of compact groups [2]: we characterize, in an abstract way, the representation theory of a compact group. The superselection structure can then be recognized as the action of a dual of a compact group on the local observables. The fields are then constructed as the cross product of the observables by this dual action.

The existence of superselection sectors in quantum field theory was first recognized by Wick, Wightmann and Wigner [3] and Haag and Kastler [4] took the important step of proposing that superselection sectors should be interpreted in terms of inequivalent irreducible representations of an abstract $C^{*}$-algebra describing the local observables.

In a local quantum theory without gravitation, the basic object is an inclusion preserving map $\mathcal{O} \rightarrow \mathfrak{A}(\mathcal{O})$ assigning to each (open) double cone $\mathcal{O}$ in spacetime the $C^{*}$-algebra generated by the observables that can be measured within $\mathcal{O}$. We refer to such a structure as a net $\mathfrak{U}$ of $C^{*}$-algebras over $\mathscr{K}$, the set of double cones. As a reflection of Einstein causality this net will satisfy local commutativity, i.e. if $\mathcal{O}_{1} \in \mathscr{K}$ is included in the spacelike complement $\mathcal{O}_{2}^{\prime}$ of $\mathcal{O}_{2} \in \mathscr{K}$ then $\mathfrak{A}\left(\mathcal{O}_{1}\right)$ and $\mathfrak{A}\left(\mathcal{O}_{2}\right)$ commute elementwise. The $C^{*}$-algebra generated by all $\mathfrak{A}(\mathcal{O})$ is called the $C^{*}$-algebra of quasilocal observables and is again denoted by $\mathfrak{A}$.

The question arises as to which irreducible representations of $\mathfrak{A}$ describe elementary excitations of the vacuum rather than, for example, some ground state with finite density. It is only these representations which are candidates for the superselection sectors of elementary particle physics. A natural approach is to impose the (relativistic) spectrum condition on the desired representations and an early attempt by Borchers [5] to study superselection sectors adopted this approach.

In a programme developed with Haag [1], we took the point of view that the representations of the $C^{*}$-algebra $\mathfrak{A}$ of quasilocal observables relevant to an

\footnotetext{
$\sqrt{1}$ In other words, we use Bose or Fermi fields with a colour index to describe parastatistics. However, quark fields in the confinement phase are no part of our formalism since our sectors are, by definition, physical sectors
} 
investigation of superselection structure should be selected by the localization properties of the associated states with respect to the vacuum state $\omega_{0}$. The relevant states $\omega$ should deviate from the vacuum essentially only in a finite region of space, i.e. $\left(\omega-\omega_{0}\right) \mid \mathfrak{A}\left(\mathcal{O}^{\prime}\right)$ should have a norm which can be made arbitrarily small by choosing a sufficiently large double cone $\mathcal{O}$. This condition led us (see [1]) to consider the following restriction on the associated representations which is a variant of the conditions considered by Brochers [5]:

$$
\pi\left|\mathfrak{A}\left(\mathcal{O}^{\prime}\right) \cong \pi_{0}\right| \mathfrak{A}\left(\mathcal{O}^{\prime}\right), \quad \mathcal{O} \in \mathscr{K}^{2}{ }^{2}
$$

Condition (1.1) says that if $\pi$ carries a charge ${ }^{3}$, this charge can be localized in any double cone $\mathcal{O}$ and we refer in this connection to localizable charges. These are the charges which can be created by localizable fields ${ }^{4}$.

It was pointed out in [1] that condition (1.1) is too stringent for Quantum Electrodynamics as it excludes all states with nonvanishing electric charge by virtue of Gauss' law. However, Buchholz and Fredenhagen [6] pointed out that (1.1) could possibly also be too stringent in massive non-Abelian gauge theories because the string or flux line attached to particles in such theories forces the localization regions of the corresponding charges to extend to spacelike infinity at least in some arbitrary direction. An investigation of charges in lattice gauge models supports this conclusion [7].

Furthermore, Buchholz and Fredenhagen [6] proved a remarkable result asserting that, in a theory with no massless excitations, a weaker form of (1.1) where double cones $\mathcal{O}$ are replaced by spacelike $\operatorname{cones}^{5} \mathscr{C}$,

$$
\pi\left|\mathfrak{A}\left(\mathscr{C}^{\prime}\right) \cong \pi_{0}\right| \mathfrak{A}\left(\mathscr{C}^{\prime}\right), \quad \mathscr{C} \in \mathscr{J}
$$

is automatically satisfied by any massive single particle representation $\pi^{6}$. They thus established a direct general link between spectral properties and localization. The validity of (1.2) shows that the effects of strings or flux lines can be localized in an arbitrary spacelike cone so that it vanishes in the spacelike complement. For this reason, charges fulfilling (1.2) but not (1.1) will be called topological charges.

The superselection structure of gauge theories with massless particles has yet to be analysed. It is clear that a weaker condition than (1.2) is needed to select the relevant representations. Furthermore, the appropriate definition of charge should correspond to equivalence classes of irreducible representations under a weaker equivalence relation than unitary equivalence since representations generated by states in Quantum Electrodynamics differing by infinitely many soft photons with

\footnotetext{
${ }^{2}$ For an unbounded region such as $\mathcal{O}^{\prime}$, the spacelike complement of $\mathcal{O}, \mathfrak{U}\left(\mathcal{O}^{\prime}\right)$ denotes the $C^{*}$-subalgebra of $\mathfrak{A}$ generated by all $\mathfrak{Q}\left(\mathcal{O}_{1}\right)$ with $\mathcal{O}_{1} \in \mathscr{K}$ and $\mathcal{O}_{1} \subset \mathcal{O}^{\prime}$.

${ }^{3}$ The term "charge" is used generically to denote the properties specific to a particular superselection sector.

${ }^{4}$ Local fields defined on a space with indefinite metric should not be regarded as localizable since the apparent localization properties get lost on passing to the associated Hilbert space of physical states.

5 Spacelike cones are defined in the Appendix; $\mathscr{J}$ denotes the set of spacelike cones.

${ }^{6}$ I.e. $\pi$ is a translation covariant representation with positive energy on a separable Hilbert space where the mass spectrum begins with a strictly positive isolated eigenvalue
} 
small total energy carry the same electric charge but are inequivalent. In fact, the investigations by Buchholz [8] make it clear that a sector is determined not just by the total electric charge but also by the asymptotic Coulomb field. The corresponding representation can be equivalent to the vacuum representation on the spacelike complement of a spacelike cone $\mathscr{C}$ only when this asymptotic Coulomb field is localized within $\mathscr{C}$. Hence for a sector with nonzero total electric charge (1.2) can at most hold for spacelike cones which are sufficiently large and point in the right general direction.

After this digression on gauge theories with massless particles, we report briefly on the analysis of the superselection structure carried out in $[1,9]$ on the basis of the selection criterion (1.1) and extended in [6] to the case of the weaker criterion (1.2). In either case the analysis leads to the following structure. To each charge corresponds a unique conjugate charge; the particle-antiparticle symmetry is a consequence of this fact. Every charge has an associated permutation symmetry characterized by a sign and an integer $d$, its statistical dimension; particle statistics is a consequence, $d$ is the order of the parastatistics and the sign distinguishes para-Bose from para-Fermi statistics. Ordinary Bose and Fermi statistics correspond to the case $d=1$. Finally, two charges can be composed because they can be created in spacelike separated regions. Regarding these separate causally disjoint acts of creating a charge as one single act defines the composed charge in a physically meaningful way?

This common superselection structure was seen as analogous to the structure of the equivalence classes of irreducible, continuous, unitary representations of a compact group. The composition of charges corresponds to the tensor product of representations, charge conjugation corresponds to passing to the conjugate representation whilst the permutation symmetry corresponds to permuting the order of factors in the tensor powers of a given representation. In view of concrete models where the observables were taken to be the gauge invariant part of a field algebra [10] it was natural to conjecture [11] that this superselection structure actually determines a compact group $G$ which then acts as the gauge group of the theory ${ }^{8}$.

We settle this conjecture in this paper by constructing a net of unobservable fields with normal Bose-Fermi commutation relations on which a compact group $G$ acts as a group of automorphisms. The observable net can be recovered as the gauge invariant part of the field net. The field net serves to describe all superselection sectors in the sense that a single irreducible vacuum representation of the field net provides a reducible representation of the observable net which is a direct sum of irreducible representations satisfying the selection criterion where each sector appears with a multiplicity equal to its statistical dimensions.

\footnotetext{
${ }^{7}$ For a more detailed survey of these results the reader is referred to the introductions to the original papers.

${ }^{8}$ In the absence of parastatistics, i.e. if every sector has $d=1$, then $G$ is Abelian and the facts which follow were already established for the case of localizable charges in [12]. The presentation of this important special case can be simplified by employing the constructions of this paper
} 
These results were conjectured on the basis of the detailed analysis of superselection structure. They are by no means "obvious" when the subject is approached from the standpoint of field-theoretical models. First, as the examples of solitonic sectors show [13], it is not a priori clear that the original fields will suffice to generate all superselection sectors. Secondly, as soon as one envisages applications to gauge theories, it is no longer clear how to pass from the original fields to fields on a physical Hilbert space with simple transformation properties under a global gauge group because of the problem of regularizing formal expressions of the form

$$
\psi(x)^{*} P \exp \left\{i e \int_{x}^{\infty} A^{\mu}\left(x^{\prime}\right) d b_{\mu}\left(x^{\prime}\right)\right\} .
$$

For this reason, even the relation between the local gauge group of the gauge theory and the global gauge group determined intrinsically by the superselection structure is unclear. Thirdly, our results are dimension dependent; a sector corresponding to a localizable charge does not necessarily exhibit permutation symmetry in two spacetime dimensions and for topological charges this phenomenon already occurs in three spacetime dimensions.

Our paper is organized as follows: we begin in Sect. 2 with an informal discussion of the problem met in trying to construct a field algebra. This is designed to help readers with a more physical background grasp the construction of field algebras as the cross product of observable algebras by the action of the dual of the compact gauge group. Readers interested only in the results could begin with Sect. 3 where we treat the case of localized charges. To express the desired properties of the field algebra and gauge group we introduce the concept of a field system with gauge symmetry (Definition 3.1) and our main result (Theorem 3.5) asserts the existence and uniqueness of a complete normal field system with gauge symmetry. We also classify abnormal commutation relations in terms of the related permutation symmetry (Theorem 3.15). A noteworthy feature of our analysis is that so little is assumed. The principal assumption is duality in the vacuum sector. Positivity of the energy plays a very indirect and rather minor role.

Section 4 gives a treatment of the basic results on superselection structure in the case of topological charges first obtained by Buchholz and Fredenhagen [6]. We include this material as it represents a substantial conceptual and technical simplification. In Sect. 5 we construct fields and a gauge group for the case of topological charges. The most noteworthy difference as compared with the case of localizable charges is that the fields can of course, in general, only be localized in spacelike cones so that we get a net $\mathscr{C} \rightarrow \mathfrak{F}(\mathscr{C})$ of von Neumann algebras over the set of spacelike cones. There is now nothing resembling a global algebra of fields because the $C^{*}$-algebra generated by the gauge invariant part of the $\mathscr{F}(\mathscr{C})$, $\mathscr{C} \in \mathscr{J}$, can no longer be expected to be algebraically intrinsic (i.e. faithfully represented on each superselection sector).

In Sect. 6 we discuss the covariance properties of the field algebras now of course assuming translation covariance and the spectrum condition in the vacuum sector and restricting our attention to covariant sectors. The spectrum condition then propagates to all sectors (cf. $[9,5]$ ). In Sect. 7 we comment on theories with 
spontaneously broken gauge symmetries. At the level of the observable algebra this manifests itself in a breakdown of duality in the vacuum sector. Instead we assume only essential duality and the superselection structure is determined by the representation theory of the unbroken part of the gauge group. Here too we construct fields carrying an action of the full gauge group. The paper concludes with an Appendix where we discuss some geometrical properties of spacelike cones needed in the analysis of topological charges.

\section{Cross Products by Dual Actions}

The existence of field algebras and gauge groups of the first kind rests upon recognizing that the superselection structure determines the action of a dual object of a compact group on the algebra of observables. The field algebra is then constructed as the cross product of the observable algebra by this dual action and the gauge group materializes as the group of automorphisms of the field algebra leaving the observable algebra pointwise fixed.

The basic results on which this construction rests have already been written up in a mathematical context $[2,14]$. All that remains to be done is to adapt this mathematical context to the different physical contexts in which we wish to apply these results and to draw the appropriate conclusions. However, to render this construction more accessible to readers with a physical background we should like to explain the basic idea of the cross product construction by concentrating on the simplest case and omitting proofs.

We will start by supposing that we have a field algebra $\mathfrak{F}$ acted on by a gauge group $G$ and then gradually uncover the problems which have to be solved if ones faces, as we do, the task of constructing $\mathfrak{F}$ and $G$ from $\mathfrak{A}$ and its superselection structure.

The simplest case is to suppose that the field algebra $\mathfrak{F}$ is generated by the observable algebra $\mathfrak{A}$ and a single multiplet $\psi_{i}=1,2, \ldots, d$ from $\mathfrak{F}$ transforming according to a faithful ${ }^{9}$, but not necessarily irreducible, unitary representation of $G$. $G$ may then be regarded as a closed subgroup of $U(d)$ and is hence a compact Lie group ${ }^{10}$. Conversely, if $G$ is a compact Lie group, the field algebra will always be generated by the observable algebra and a single multiplet of fields. This simplest case therefore includes most of the gauge groups one would envisage.

We may also assume that the multiplet of fields transforms according to a representation of determinant one so that $G$ is then a subgroup of $S U(d)$. If necessary, we may, for example, replace the original faithful representation of $G$ by the direct sum of this representation and its conjugate representation. As we shall see, the choice $G \subset S U(d)$ is essential if the simple cross product construction is to work. The basic reason for this is that, if $G \subset S U(d)$, every irreducible representation of

\footnotetext{
${ }^{9}$ Our gauge group $G$ acts faithfully on $\mathfrak{\&}$ by definition. This might, for example, mean using $S U(3) / \mathbf{Z}_{3}$ rather than $S U(3)$, but has the invaluable merit of making $G$ unique up to isomorphism.

${ }^{10} \mathrm{G}$ need not, of course, be a connected Lie group and it might even be a $o$-dimensional compact Lie group, i.e. finite group
} 
$G$ is a subrepresentation of some tensor power of the defining representation so that the set of such subrepresentations is automatically self-conjugate.

In view of the correspondence between irreducible representations of $G$ and superselection sectors which can be established in the presence of a field algebra, cf. [10; Theorem 3.6] and Eq. (3.27), the simple case we are considering can be characterized intrinsically by saying that the superselection structure is finitely generated, i.e. every sector is obtained by reducing the products of powers of a finite number of basic sectors.

In order to recognize what is intrinsic in the structure of the *-algebra generated by $\mathfrak{A}$ and a basic multiplet, the first important step is to realize [11; Sect. 3] that this multiplet can be chosen to satisfy the simple algebraic relations

$$
\begin{aligned}
& \psi_{i}^{*} \psi_{j}=\delta_{i j} I \\
& \sum_{i} \psi_{i} \psi_{i}^{*}=I
\end{aligned}
$$

Their linear span is then a $d$-dimensional Hilbert space $H$ with a scalar product given by

$$
\left(\psi, \psi^{\prime}\right) I:=\psi^{*} \psi^{\prime}, \quad \psi, \psi^{\prime} \in H .
$$

Next, it is easily seen that setting

$$
\rho(A):=\sum_{i} \psi_{i} A \psi_{i}^{*}
$$

must define a unit-preserving endomorphism of $\mathfrak{A}$. In fact, $\rho(I)=I$ follows from (2.2), $\rho(A B)=\rho(A) \rho(B)$ from (2.1) and $\rho\left(A^{*}\right)=\rho(A)^{*}$ from (2.4) itself so that it only remains to understand why $\rho(A) \in \mathfrak{A}$. However, whatever subgroup of $S U(d) G$ turns out to be, the right-hand side of (2.4) will be invariant under $G$ and hence must be an element of $\mathfrak{A}$. The endomorphism $\rho$ is part of the given data of our cross product construction and has in our context an intrinsic physical meaning ${ }^{11}$. If the multiplet transforms irreducibly under $G, \rho$ generates from the vacuum representation some superselection sector. In general, it generates some finite direct sum of sectors.

The next point to realize is that

$$
\psi A=\rho(A) \psi, \quad \psi \in H .
$$

This follows by multiplying (2.4) on the right with $\psi$ and using the fact that the $\psi_{i}$ form an orthonormal basis of $H$. Thus (2.5) is the commutation relation between our basic multiplet and $\mathfrak{A}$.

The problem we have to solve now begins to take a precise shape: given $\mathfrak{A}$ and the basic endomorphism $\rho$, define a *-algebra generated by $\mathfrak{U}$ and $d$ elements $\psi_{i}, \psi_{2}, \ldots, \psi_{d}$ satisfying (2.1), (2.2) and (2.5). This *-algebra should carry an action of a group $G \subset S U(d)$ by automorphisms leaving the subspace $H$ globally stable and having $\mathfrak{A}$ as the fixed point subalgebra.

${ }^{11}$ This will become clear in Sect. 3, cf. Eq. (3.13) 
Inside our *-algebra will be the *-subalgebra generated by the multiplet $\psi_{i}, i=1,2, \ldots, d$ which we denote by ${ }^{\circ} \mathcal{O}_{d}$ and whose structure is entirely determined by (2.1) and (2.2) in the sense that there can be no further independent relations within our ${ }^{*}$-algebra. In fact Cuntz showed [15] that the algebra ${ }^{\circ} \mathcal{O}_{d}$ can be completed in a unique way to give a $C^{*}$-algebra $\mathcal{O}_{d}$, the Cuntz algebra.

It is very easy to compute within the algebra ${ }^{\circ} \mathcal{O}_{d}$ : every element of this algebra can be written as a finite linear combination of elements of the form

$$
\psi_{i_{1}} \psi_{i_{2}} \cdots \psi_{i_{r}} \psi_{j_{s}}^{*} \cdots \psi_{j_{2}}^{*} \psi_{j_{1}}^{*}
$$

and (2.1) tells us how such elements can be multiplied. Now if we set

$$
\mathrm{S}:=d !^{-1 / 2} \sum_{p \in \mathbf{P}_{d}} \operatorname{sign}(p) \psi_{p(1)} \psi_{p(2)} \cdots \psi_{p(d)}
$$

where $\mathbf{P}_{d}$ denotes the permutation group on $d$ elements, then a computation within ${ }^{\circ} \mathcal{O}_{d}$ shows that (cf. [17; Lemma 2.2])

$$
\psi_{i}^{*}=(-1)^{d-1} \sqrt{d} S^{*} \hat{\psi}_{i},
$$

where

$$
\hat{\psi}_{i}:=((d-1) !)^{-1 / 2} \sum_{p \in \mathbf{P}_{d}(l)} \operatorname{sign}(p) \psi_{p(2)} \psi_{p(3)} \cdots \psi_{p(d)}
$$

and $\mathbf{P}_{d}(i)$ denotes the subset of permutations with $p(1)=i$.

Now under a unitary transformation in $H$, the isometry $S$ gets multiplied by the determinant of that unitary transformation. Therefore, whatever subgroup of $S U(d) G$ turns out to be, $S$ will be a $G$-invariant element of our *-algebra and hence equal to an isometry $R$ of $\mathfrak{A}$. This isometry $R$ is also part of the given data of the cross product construction. Actually, $R$ is given as an element of $\mathfrak{A}$ expressing the "special conjugate property" of $\rho$ [14; Sect. 4], cf. Lemma 3.7. It allows us to complete the commutation relations between ${ }^{\circ} \mathcal{O}_{d}$ and $\mathfrak{A}$ in our $*_{\text {-algebra since }}$ (2.8), (2.9) and (2.5) imply

$$
\psi_{i}^{*} A=(-1)^{d-1} \sqrt{d} R^{*} \rho^{d-1}(A) \hat{\psi}_{i} .
$$

Bearing (2.6) in mind, we see that (2.5) and (2.10) allow us to write every element of our *-algebra in the form

$$
\sum_{n} A_{n} C_{n}, \quad A_{n} \in \mathfrak{U}, \quad C_{n} \in \mathcal{O}^{\circ}
$$

and tell us how multiplication and the adjoint are to be computed in our *-algebra.

At first sight, it might seem as if our *-algebra has been constructed and that only more technical problems such as the completion to give a $C^{*}$-algebra remain. However, the trouble is that the expression (2.11) for an element of our *-algebra is highly non-unique: as a linear space our algebra is not just the tensor product of $\mathfrak{A}$ with ${ }^{\circ} \mathcal{O}_{d}$ as (2.11) might suggest. Indeed we have already made essential use of the fact that the isometry $R \in \mathfrak{A}$ is identified with $S \in \in^{\circ} \mathcal{O}_{d}$ in our *-algebra. Since we want $\mathfrak{U}$ to be the gauge-invariant part of our ${ }^{*}$-algebra, the ${ }^{*}$-algebra ${ }^{\circ} \mathcal{O}_{G}$ of gauge-invariant elements of ${ }^{\circ} \mathcal{O}_{d}$ must be identified with a ${ }^{*}$-subalgebra of $\mathfrak{U}$. It is convenient to express this identification as a morphism $\mu:{ }^{\circ} \mathcal{O}_{\boldsymbol{G}} \rightarrow \mathfrak{U}$ of ${ }^{*}$-algebras 
so that in particular $\mu(S)=R$. We now identify

$$
A(X C)=(A \mu(X)) C, \quad X \in^{\circ} \mathcal{O}_{G}, \quad A \in \mathfrak{A}, \quad C \epsilon^{\circ} \mathcal{O}_{d}
$$

so our *-algebra, instead of being the tensor product of $\mathfrak{A}$ and ${ }^{\circ} \mathcal{O}_{\boldsymbol{d}}$ as a linear space, is the ${ }^{\circ} \mathcal{O}_{G}$-module tensor product of $\mathscr{U}$ and ${ }^{\circ} \mathcal{O}_{d}$. The multiplication and *-operation are defined using (2.5) and (2.10) as before. Of course, it has to be checked that these operations are consistent with the identification (2.12). It then immediately becomes apparent that $\mu$ and $\rho$ must satisfy certain compatibility conditions: if we define an endomorphism $\sigma$ of ${ }^{\circ} \mathcal{O}_{G}$ by setting

$$
\sigma(X):=\sum_{i} \psi_{i} X \psi_{i}^{*}
$$

then we can only identify $X$ and $\mu(X)$ in our *-algebra if $\mu \sigma(X)=\rho \mu(X), X \in{ }^{\circ} \mathcal{O}_{G}$, i.e. if

$$
\mu \circ \sigma=\rho \circ \mu .
$$

It turns out that rather more is needed to make the construction work. If we consider the spaces of intertwining operators between powers of these endomorphisms,

$$
\begin{aligned}
& \left(\rho^{r}, \rho^{s}\right):=\left\{T \in \mathfrak{A}: T \rho^{r}(A)=\rho^{s}(A) T, A \in \mathfrak{U}\right\}, \\
& \left(\sigma^{r}, \sigma^{s}\right):=\left\{X \in{ }^{\circ} \mathcal{O}_{G}: X \sigma^{r}(Y)=\sigma^{s}(Y) X, Y \in{ }^{\circ} \mathcal{O}_{G}\right\},
\end{aligned}
$$

then we need

$$
\mu\left(\sigma^{r}, \sigma^{s}\right) \subset\left(\rho^{r}, \rho^{s}\right), \quad r, s \in \mathbf{N}_{0} .
$$

In particular, it is easily checked that $S \in\left(l, \sigma^{d}\right)$ so that we must have $\mu(S)=R \in\left(l, \rho^{d}\right)$. Here $l$ denotes the identity automorphisms $\sigma^{0}$ and $\rho^{0}$ respectively.

This leads us to define an action of ${ }^{\circ} \mathcal{O}_{G}$ on a unital ${ }^{*}$-algebra $\mathscr{A}$ to be a pair consisting of an endomorphism $\rho$ of $\mathscr{A}$ and a morphism $\mu:{ }^{\circ} \mathcal{O}_{G} \rightarrow \mathscr{A}$ of ${ }^{*}$-algebras with unit satisfying (2.14) and (2.15). Under these circumstances, the construction sketched above can be carried through and leads to a $*$-algebra $\mathscr{M}$ called the cross product of $\mathscr{A}$ by the action $\mu$. The details may be found in Sect. 2 of [14]. Furthermore, as we might anticipate from the discussion above, $\mathscr{M}$ comes equipped with an action $\alpha$ of $G$ such that the fixed-point subalgebra is $\mathscr{A}$.

When $\mathscr{A}$ is a $C^{*}$-algebra, the resulting *-algebra may be completed in a natural way to form a $C^{*}$-algebra denoted $\mathscr{A} \otimes_{\mu} \mathcal{O}_{d}$ called the cross product of $\mathscr{A}$ by the action $\mu$ which comes equipped with an action $\alpha$ of $G$ with fixed-point algebra $\mathscr{A}$. The details may be found in Sect. 3 of [14].

Again it might seem as if our problems are over but, if we return to our original task of constructing the field algebra $\mathfrak{F}$ from the observable algebra $\mathfrak{A}$ and its superselection structure, we note that all the above construction says is that if we knew the gauge group $G$ in the shape of the appropriate action $\mu: \mathcal{O}_{G} \rightarrow \mathfrak{U}$ then we could construct the field algebra $\mathfrak{F}$. Conversely, if we knew the field algebra $\mathfrak{F}$ then we could construct $G$ as the group of automorphisms of $\mathfrak{F}$ leaving $\mathfrak{A}$ pointwise invariant and $\mu: \mathcal{O}_{G} \rightarrow \mathfrak{A}$ is just the restriction of the embedding $\mathcal{O}_{d} \subset \mathscr{F}$ to the gauge-invariant elements. However, we know neither $\mathfrak{F}$ not $G$.

The way out of this dilemma is to begin with the group $S U(d)$ that we do 
know $^{12}$. The algebra $\mathcal{O}_{S U(d)}$ is generated by the isometry $S$ of $(2.7)$ and the unitary operator

$$
\vartheta:=\sum_{i, j} \psi_{i} \psi_{j} \psi_{i}^{*} \psi_{j}^{*}
$$

More precisely, it is the smallest $\sigma$-invariant $C^{*}$-subalgebra of $\mathcal{O}_{d}$ containing $\vartheta$ and $S$ (see [17; Lemma 3.7]). This is closely related to the statement that all invariants for $S U(d)$ are built up from the scalar product and the determinant. $\mu(\vartheta)=\varepsilon$ is also part of the data of the cross product construction. The operator $\varepsilon$ is also known in the field-theoretic context; it is the basic statistics operator $\varepsilon(\rho, \rho)$ associated with $\rho$ in the case of para-Bose statistics or its "Bosonized" version $\hat{\varepsilon}(\rho, \rho)$ in the general case (cf. Sect. 3).

One can now show $[17$; Corollary 4.4$]$ that there is a unique action $\mu: \mathcal{O}_{S U(d)} \rightarrow \mathfrak{U}$ such that $\mu(\vartheta)=\varepsilon, \mu(S)=R$ with $\mu \circ \sigma=\rho \circ \mu$. Hence there is no obstacle to defining the cross product of $\mathfrak{A}$ by this action. This construction does not yield the field algebra directly since it is only the $S U(d)$-invariant elements of $\mathcal{O}_{d}$ which have been identified with their counterparts in $\mathfrak{A}$. If $G$ is a proper subgroup of $S U(d)$, the algebra $\mathfrak{U} \otimes_{\mu} \mathcal{O}_{d}$ is thus too large and has a non-trivial centre. The field algebra $\mathfrak{F}$ is now the quotient of $\mathfrak{A} \otimes_{\mu} \mathcal{O}_{d}$ by an ideal which kills the centre of $\mathfrak{A} \otimes_{\mu} \mathcal{O}_{d}$, see [14; Lemma 4.4]. In retrospect, this field algebra $\mathfrak{F}$ is just the cross product of $\mathfrak{A}$ by the dual action of a closed subgroup $G$ of $S U(d)$. $G$ may be identified as the group of automorphisms of $\mathfrak{F}$ leaving $\mathfrak{U}$ pointwise fixed and it is that subgroup of $S U(d)$ whose canonical action on $\mathfrak{A} \otimes_{\mu} \mathcal{O}_{d}$ leaves the chosen ideal invariant.

The properties of this indirect cross product construction are the content of the pivotal Theorem 4.1 of [14]. The uniqueness is stated as Theorem $4.7 \mathrm{ibid}$. By virtue of the identification of $\varepsilon$ with $\hat{\varepsilon}(\rho, \rho)$, the field algebra has normal Bose-Fermi commutation relations.

The reader will discover that we do not make direct use of [14; Theorem 4.1] in the sequel but rather of a more general result [14; Theorem 5.1] which is derived from it. There are two good reasons for this. First, it allows us to treat the general case where $G$ turns out to be an arbitrary compact group, and secondly we do not have to single out a particular $\rho$ but can treat them all on an equal footing. Finally, since we want to construct the field algebra $\mathfrak{F}$ in its vacuum representation, it turns out to be more convenient to use a spatial version of the cross product [14; Sect. 6] derived in a rather simple fashion from [14; Theorem 5.1].

\section{Localizable Charges}

In this section we present our basic results on the existence of a field algebra and a gauge group describing the superselection structure when the charges in question can be localized in any double cone ${ }^{13}$. Quite apart from the intrinsic importance

\footnotetext{
12 The integer $d$ is uniquely determined by $\rho$, it is the statistical dimension of $\rho$.

13 The analysis of $[1,9]$ only demands that the charges can be localized in all translates of some double cone. We have desisted from working in this generality here so as to keep the ideas as simple as possible and because there is no evidence for a minimal length associated with charges
} 
of this case, this has the merit of not obscuring the basic construction with technicalities which arise in the case of topological charges. For the same reason, we will begin this section by assuming that the observable algebra satisfies duality for double cones in the vacuum sector:

$$
\pi_{0}\left(\mathfrak{A}\left(\mathcal{O}^{\prime}\right)\right)^{\prime}=\pi_{0}(\mathfrak{A}(\mathcal{O})), \quad \mathcal{O} \in \mathscr{K} .
$$

This rules out the possibility of spontaneously broken gauge symmetries but we shall allow for this possibility later by discussing a weaker form of (3.1), essential duality. It has been shown by Bisognano and Wichmann [16] that essential duality holds whenever the local algebras are generated by an underlying Wightman field theory. It is a noteworthy feature of the analysis that the existence of a gauge group and a field net describing the superselection structure depends only on duality and two further assumptions, first that the vacuum representation ${ }^{14} \pi_{0}$ is faithful and irreducible and that $\mathscr{H}_{0}$, the vacuum Hilbert space is separable ${ }^{15}$ and secondly

Property B. If $E \in \mathfrak{A}(\mathcal{O})$ is a non-zero projection then, for any $\mathcal{O}_{1}$ containing the closure of $\mathcal{O}$, there is an isometry $W \in \mathfrak{A}\left(\mathcal{O}_{1}\right)$ with $W W^{*}=E$ and $W^{*} W=I$ (i.e. the projection $E$ is equivalent to $I$ in $\left.\mathfrak{A}\left(\mathcal{O}_{1}\right)\right)$.

Property B was derived by Borchers [18] from standard structural assumptions of Quantum Field Theory, viz. positivity of the energy, locality and weak additivity. Of course, as we shall see, certain properties of the field net such as the Reeh-Schlieder property, additivity and covariance depend on the corresponding property of the observable net.

As mentioned in the introduction, studying localizable charges means more precisely investigating the property of the set of representations satisfying (1.1). Such an investigation was carried out in [1,9] and the concept of statistics or permutation symmetry played a fundamental role. To every representation $\pi$ satisfying (1.1), we can associate [1; Sect. V] a unitary equivalence class of representations of the permutation group $\mathbf{P}_{n}$ on $n$ symbols, $n=2,3, \ldots$ If there are no restrictions on the Young tableaux involved $\pi$ is said to have infinite statistics. If the Young tableaux involved are those whose columns (rows) have length $\leqq d$, then $\pi$ is said to have para-Bose (para-Fermi) statistics of order $d$ (the statistical dimension of $\pi$ ). It is a remarkable consequence of the general assumptions above that if $\pi$ is irreducible these are the only possibilities. If $\pi$ is an infinite direct sum of representations with finite (i.e. not infinite) statistics, then $\pi$ has infinite statistics. The converse is expected to be true and results in this direction may be found in the Appendix of [9] and in a paper of Fredenhagen [19]. The statistics of $\pi$ depend, of course, only on the unitary equivalence class of $\pi$ and they are hence a property of the underlying charges.

\footnotetext{
${ }^{14}$ Should the vacuum representation fail to be unique then the field algebra and gauge group are to be understood as relative to the chosen vacuum representation. Faithful means that $\pi_{0}(A)=0$ implies $A=0$.

${ }^{15}$ But see the remark following Lemma 3.11
} 
Under the assumptions stated above, our main theorem, Theorem 3.5 below, asserts the existence and uniqueness of an irreducible net of local field algebras with normal commutation relations acted on by a compact group of internal symmetries (the group of global gauge transformations) where the underlying Hilbert space contains all superselection sectors. Namely, the local field algebras are generated by field operators which lead from the vacuum Hilbert subspace (vacuum sector) to Hilbert subspaces associated with each equivalence class of irreducible representations of $\mathfrak{U}$ satisfying (1.1) and having finite statistics and which either commute or anticommute at spacelike separations depending, in the usual way, on the para-Bose or para-Fermi character of the sectors involved.

Before stating our main results as theorems, it is convenient to specify the desired structural properties in the form of definitions.

3.1 Definition. Let $\pi_{0}$ denote the vacuum representation of the net $\mathfrak{A}$ of local observables acting on the Hilbert space $\mathscr{H}_{0}$. A field system with gauge symmetry, $\{\pi, G, \mathfrak{F}\}$, consists of a representation $\pi$ of $\mathfrak{U}$ on a Hilbert space $\mathscr{H}$ containing $\pi_{0}$ as a subrepresentation on $\mathscr{H}_{0} \subset \mathscr{H}$, a (strongly) compact group $G$ of unitaries on $\mathscr{H}$ leaving $\mathscr{H}_{0}$ pointwise fixed and a net $\mathcal{O} \in \mathscr{K} \rightarrow \mathscr{F}(\mathcal{O})$ of von Neumann algebras acting on $\mathscr{H}$ (the field net) such that

$\alpha)$ the $g \in G$ induce automorphisms $\alpha_{g}$ of $\mathfrak{F}(\mathcal{O})$ with $\pi(\mathfrak{U}(\mathcal{O}))$ as fixed-point algebra, $\mathcal{O} \in \mathscr{K}$

$\beta)$ the field net $\mathfrak{F}$ is irreducible;

$\gamma) \mathscr{H}_{0}$ is cyclic for $\mathfrak{F}(\mathcal{O}), \mathcal{O} \in \mathscr{K}^{16}$

$\delta)$ the fields are local relative to the observables, i.e. $\mathfrak{F}\left(\mathcal{O}_{1}\right)$ and $\pi\left(\mathfrak{A}\left(\mathcal{O}_{2}\right)\right)$ commute elementwise whenever $\mathcal{O}_{1}$ and $\mathcal{O}_{2}$ are spacelike separated.

Condition $\delta$ ) does not, of course, imply normal commutation relations so we make a further definition:

3.2 Definition. A field system with gauge symmetry $\{\pi, G, \mathfrak{F}\}$ is normal if there is an element $k$ in the centre of $G$ with $k^{2}=e$ such that the net $\mathfrak{F}$ obeys graded local commutativity for the $\mathbf{Z}_{2}$-grading defined by $k$. In other words, if $\mathcal{O}_{1}, \mathcal{O}_{2} \in \mathscr{K}$ are spacelike separated and $F \in \mathfrak{F}\left(\mathcal{O}_{1}\right), F^{\prime} \in \mathfrak{F}\left(\mathcal{O}_{2}\right)$ then

$$
F_{+} F_{+}^{\prime}=F_{+}^{\prime} F_{+}, \quad F_{+} F_{-}^{\prime}=F_{-}^{\prime} F_{+}, \quad F_{-} F_{-}^{\prime}=-F_{-}^{\prime} F_{-},
$$

where

$$
F_{ \pm}=\frac{1}{2}\left(F \pm \alpha_{k}(F)\right)
$$

The field systems defined so far do not necessarily describe all superselection sectors, for example $\left\{\pi_{0},\{I\}, \mathfrak{A}\right\}$ is always a normal field system with (trivial) gauge symmetry. This motivates the next definition:

3.3 Definition. A field system with gauge symmetry $\{\pi, G, \mathfrak{F}\}$ is complete if each equivalence class of irreducible representations of $\mathfrak{A}$ satisfying (1.1) and having finite

\footnotetext{
16 This reflects the fact that (1.1) holds for all $\mathscr{O} \in \mathscr{K}$ and would need modification if there were a minimal length associated with charges
} 
statistics is realized as a subrepresentation of $\pi$, i.e. $\pi$ describes all the relevant superselection sectors.

Finally, we need a notion of equivalence of two field systems so that we can claim our construction is unique up to equivalence.

3.4 Definition. Two field systems with gauge symmetry $\left\{\pi_{i}, G_{i}, \mathfrak{F}_{i}\right\}, i=1,2$ are equivalent if there is a unitary operator $W: \mathscr{H}_{1} \rightarrow \mathscr{H}_{2}$ such that

$$
\begin{aligned}
W \pi_{1}(A) & =\pi_{2}(A) W, \quad A \in \mathfrak{A}, \\
W G_{1} & =G_{2} W, \\
W \mathfrak{F}_{1}(\mathcal{O}) & =\mathfrak{F}_{2}(\mathcal{O}) W, \quad \mathcal{O} \in \mathscr{K} .
\end{aligned}
$$

We can now state our main results on the existence, uniqueness and principal properties of field systems.

3.5 Theorem. Let $\mathfrak{A}$ be a net of local observables satisfying Property $B$ and suppose that duality (3.1) holds in the faithful, irreducible vacuum representation $\pi_{0}$ acting on a separable Hilbert space $\mathscr{H}_{0}$. Then there exists a complete normal field system with gauge symmetry and this system is unique up to equivalence.

3.6 Theorem. Let $\{\pi, G, \mathfrak{F}\}$ be a field system with gauge symmetry then

a) $\pi(\mathfrak{U})^{\prime} \cap \mathfrak{F}=C I^{17}$

b) an automorphism $\gamma$ of the $C^{*}$-algebra $\mathfrak{F}$ is a gauge automorphism, i.e. $\gamma=\alpha_{g}$ for some $g \in G$ if and only if $\gamma$ acts trivially on $\pi(\mathfrak{U})$.

c) $\pi(\mathfrak{U})^{\prime}=G^{\prime \prime}$ and

$$
\pi=\bigoplus_{\xi} d(\xi) \pi_{\xi}
$$

where the $\pi_{\xi}$ are inequivalent irreducible representations of $\mathfrak{U}$ fulfilling (1.1) and having parastatistics of finite order $d(\xi)^{18}$.

If furthermore the system is normal then

d) The grading of $\mathscr{H}$ defined by $k \in G$ corresponds precisely to the distinction between para-Bose and para-Fermi statistics, i.e. if $\Phi \in \mathscr{H}_{\xi}$, the Hilbert space of $\pi_{\xi}$, then $k \Phi= \pm \Phi$ according as $\pi_{\xi}$ has para-Bose or para-Fermi statistics.

e) The net $\mathfrak{F}$ satisfies twisted duality, i.e. if we set

$$
\mathfrak{F}^{t}(\mathcal{O})=V \mathfrak{F}(\mathcal{O}) V^{*}, \quad V=2^{-1 / 2}(I+i k),
$$

then

$$
\mathfrak{F}^{t}(\mathcal{O})=\mathfrak{F}\left(\mathcal{O}^{\prime}\right)^{\prime}, \quad \mathcal{O} \in \mathscr{K} .
$$

Before going on to prove these results we make some general remarks. First, the existence of a gauge group is critically dependent on the existence of statistics (permutation symmetry) and we shall later comment briefly on the exceptional situation of localizable charges in a 2-dimensional spacetime. Secondly, the algebraic structure of the field net is unique in Theorem 3.5 precisely because we

17 The symbol $\mathfrak{F}$ is used to denote both the net and, as here, the $C^{*}$-algebra generated by the net.

18 When the system is complete the sum runs by definition over all classes $\xi$ of such representation 
are insisting on normal commutation relations for the fields. The unitary equivalence class of $\pi$ is the same for any complete field system with gauge symmetry, i.e. it is independent of the choice of commutation relations. Note that the representation $\pi_{\xi}$ in (3.7) appears with multiplicity $d(\xi)$; the price to be paid for describing parastatistics $(d(\xi)>1)$ in terms of a field net constructed from parafields (trilinear commutation relations) is that the field net will not admit a gauge symmetry, cf. [20]. Finally, it is worth noting that the algebraic structure of the complete normal field system is independent of $\pi_{0}$ : if there were another vacuum representation $\hat{\pi}_{0}$ also acting faithfully on a separable Hilbert space and fulfilling duality (3.1) (so that, in particular, $\pi_{0}$ and $\hat{\pi}_{0}$ would be locally unitary equivalent) and if $\{\hat{\pi}, \hat{G}, \hat{F}\}$ were a complete normal field system with gauge symmetry relative to $\hat{\pi}_{0}$, there would be an isomorphism $\gamma$ of $\mathfrak{F}$ onto $\hat{\mathfrak{F}}$ such that

$$
\begin{aligned}
\gamma\left(\pi_{0}(A)\right) & =\hat{\pi}_{0}(A), \quad A \in \mathfrak{U}, \\
\gamma(\mathfrak{F}(\mathcal{O})) & =\hat{\mathfrak{F}}(\mathcal{O}), \quad \mathcal{O} \in \mathscr{K}, \\
\gamma \circ \alpha_{G} & =\alpha_{\hat{G}} \circ \gamma .
\end{aligned}
$$

We next recapitulate those results of $[1,9]$ which are needed as input here, introducing at the same time the relevant notation. These results are used to prove Lemma 3.7 below which in turn allows us to employ the results on cross products contained in Sects. 5 and 6 of [14].

a) The set of representations satisfying (1.1) is closed under (finite) direct sums and subrepresentations [1; Lemma 2.5]. It is here that Property B plays a crucial role.

b) Every representation $\pi$ satisfying (1.1) is equivalent to a representation of the form $\pi_{0}{ }^{\circ} \rho$, where $\rho$ is an endomorphism of $\mathfrak{U}$ satisfying

$$
\rho(A)=A, \quad A \in \mathfrak{U}\left(\mathcal{O}^{\prime}\right) .
$$

This is proved in [1; Proposition 2.1] and such an endomorphism is termed a localized morphism with localization region or support in $\mathcal{O}$.

The set of localized morphisms for which $\pi_{0} \circ \rho$ satisfies (1.1) and has finite statistics will be denoted by $\Delta$ and we write $\Delta(\mathcal{O})$ for the subset localized in $\mathcal{O}$.

c) If $\rho, \rho^{\prime} \in \Delta$ and $T$ interwines the representations $\pi_{0}{ }^{\circ} \rho$ and $\pi_{0}{ }^{\circ} \rho^{\prime}$, i.e. if

$$
T \rho(A)=\rho^{\prime}(A) T, \quad A \in \mathfrak{U},
$$

then $T \in \mathfrak{A}$. This is an elementary consequence of (3.1) and (3.13), cf. [1; Sect. 4]. It is here and in the proof of b) that duality plays a role. We write $T \in\left(\rho, \rho^{\prime}\right)$ to express (3.14) and $1_{\rho}$ to denote the unit $I$ considered as an element of $(\rho, \rho)$. If $T^{\prime} \in\left(\rho^{\prime}, \rho^{\prime \prime}\right)$, we write $T^{\prime} \circ T$ to denote $T^{\prime} T$ considered as an element of $\left(\rho, \rho^{\prime \prime}\right)$. With this composition law we get a category $\mathscr{T}$ whose objects are the elements of $\Delta$ and whose arrows are the intertwiners. $\Delta$ is closed under direct sums and subobjects [1; Sect. VI]; this reexpresses a) but for representations of the form $\pi_{0}{ }^{\circ} \rho, \rho \in \Delta$. Every element of $\Delta$ is a finite direct sum of irreducibles from $\Delta$.

d) If $\rho_{1}, \rho_{2} \in \Delta$, then $\rho_{1} \rho_{2} \in \Delta$ [1; Corollary 6.8]. Given $T_{i} \in\left(\rho_{i}, \rho_{i}^{\prime}\right)$, we write $T_{1} \times T_{2}$ to denote $T_{1} \rho_{1}\left(T_{2}\right)=\rho_{1}^{\prime}\left(T_{2}\right) T_{1}$ considered as an element of $\left(\rho_{1} \rho_{2}, \rho_{1}^{\prime} \rho_{2}^{\prime}\right)$. 
e) For each $\rho_{1}, \rho_{2} \in \Delta$ there is a unitary $\hat{\varepsilon}\left(\rho_{1}, \rho_{2}\right) \in\left(\rho_{1} \rho_{2}, \rho_{2} \rho_{1}\right)$ satisfying

$$
\begin{gathered}
\hat{\varepsilon}\left(\rho_{1}^{\prime}, \rho_{2}^{\prime}\right) \circ T_{1} \times T_{2}=T_{2} \times T_{1} \circ \hat{\varepsilon}\left(\rho_{1}, \rho_{2}\right), \quad T_{i} \in\left(\rho_{i}, \rho_{i}^{\prime}\right), \quad i=1,2, \\
\hat{\varepsilon}\left(\rho_{1}, \rho_{2}\right) \circ \hat{\varepsilon}\left(\rho_{2}, \rho_{1}\right)=1_{\rho_{2} \rho_{1}}, \\
\hat{\varepsilon}(l, \rho)=\hat{\varepsilon}(\rho, l)=1_{\rho}, \\
\hat{\varepsilon}\left(\rho_{1} \rho_{2}, \rho_{3}\right)=\hat{\varepsilon}\left(\rho_{1}, \rho_{3}\right) \times 1_{\rho_{2}} \circ 1_{\rho_{1}} \times \hat{\varepsilon}\left(\rho_{2}, \rho_{3}\right) .
\end{gathered}
$$

See Eqs. (5.5)-(5.7) of [21]. Here $\imath$ denotes the identity automorphism of $\mathfrak{A} . \hat{\varepsilon}\left(\rho_{1}, \rho_{2}\right)$ is just the "Bosonized" version of the operator $\varepsilon_{\tau}\left(\rho_{1}, \rho_{2}\right)$ in [1; Theorem 4.2] which we shall denote by $\varepsilon\left(\rho_{1}, \rho_{2}\right)$ and which also satisfies the analogues of (3.15)-(3.18). $\varepsilon$ is uniquely characterized by the fact that $\varepsilon\left(\rho_{1}, \rho_{2}\right)=I$ whenever $\rho_{1}$ and $\rho_{2}$ are spacelike separated. If $\pi_{0} \circ \rho_{1}$, and $\pi_{0}{ }^{\circ} \rho_{2}$ are irreducible then

$$
\hat{\varepsilon}\left(\rho_{1}, \rho_{2}\right)= \pm \varepsilon\left(\rho_{1}, \rho_{2}\right) \text {, }
$$

where the minus sign is to be taken if and only if the sectors $\pi_{0}{ }^{\circ} \rho_{1}$ and $\pi_{0}{ }^{\circ} \rho_{2}$ both have para-Fermi statistics. Thus if $\rho_{1}$ and $\rho_{2}$ are also spacelike separated we simply have

$$
\hat{\varepsilon}\left(\rho_{1}, \rho_{2}\right)= \pm 1_{\rho_{1} \rho_{2}} .
$$

It is the use of $\hat{\varepsilon}$ in our construction which ensures that the field net has normal Bose-Fermi commutation relations.

f) Given $\rho \in \Delta$ there exists a $\bar{\rho} \in \Delta$ and an $R \in(l, \bar{\rho} \rho)$ such that

$$
\begin{aligned}
& \bar{R}^{*} \times 1_{\rho}^{\circ} \circ 1_{\rho} \times R=1_{\rho}, \\
& R^{*} \times 1_{\bar{\rho}}^{\circ} 1_{\bar{\rho}} \times \bar{R}=1_{\bar{\rho}},
\end{aligned}
$$

where $\bar{R}=\hat{\varepsilon}(\bar{\rho}, \rho) \circ R$, see [9; Theorem 3.3] and [21; Theorem 5.2].

The properties d),e) and f) express the three main elements of superselection structure mentioned in the Introduction namely charge composition, permutation symmetry (statistics) and charge conjugation and correspond to the main structural properties of the category $\mathrm{U}(G)$ of finite dimensional, continuous, unitary representations of a compact group $G^{19} . \mathscr{T}$ is a strict symmetric monoidal $C^{*}$-category with conjugates in the terminology of [2; Sect. 1]. Furthermore $\mathscr{T}$ is closed under direct sums and subjects (cf. c) above) and $(l, l)=\mathbf{C} 1$, since $\pi_{0}$ is irreducible.

The main theorem of [2] could now be invoked to show that $\mathscr{T}$ is isomorphic to a category of the type $\mathbf{U}(G)$ for a compact group $G$ unique up to isomorphism. However, we prefer to make our discussion independent of Sects. 4-6 of [2]. The computations of Sect. 2 of [2] are essentially contained in [1,9 and 21] but the computations in Sect. 3 of [2] are new and are needed to prove:

3.7 Lemma. The pair $(\Delta, \hat{\varepsilon})$ is a permutation symmetric semigroup of unital endomorphisms of $\mathfrak{A}$ and is specially directed in the sense of [14; Sect. 5].

\footnotetext{
19 The reason for using $\hat{\varepsilon}$ rather than $\varepsilon$ is precisely so as to have the conjugate equations, (3.21), (3.22), in the form holding in $\mathbf{U}(G)$
} 
Proof. e) above expresses what is meant by a permutation symmetry. By [2; Lemma 3.7], if $\rho$ is a special object of $\mathscr{T}$ there is an isometry $R \in\left(l, \rho^{d}\right)$ with

$$
\begin{gathered}
R^{*} \rho(R)=(-1)^{d-1} d^{-1} I, \\
R R^{*}=d !^{-1} \sum_{p \in \mathbf{P}_{d}} \operatorname{sign}(p) \hat{\varepsilon}_{\rho}(p),
\end{gathered}
$$

where $\hat{\varepsilon}_{\rho}$ is the unitary representation of the group $\mathbf{P}_{\infty}$ of finite permutations of the integers associated with $\rho$ by the theorem in the Appendix of [14]. Actually, by [1; Proposition 4.4] there is a representation $\varepsilon_{\rho}$ of $\mathbf{P}_{\infty}$ associated with $\varepsilon$ and $\hat{\varepsilon}_{\rho}$ is just its "Bosonized" version; $d$ is the statistical dimension of $\rho$. Equations (3.23) and (3.24) express what is meant by $\rho$ being special in the sense of [14; Sect. 5]. Since $\Delta$ has direct sums, subobjects and conjugates, given $\rho_{1}, \rho_{2}, \ldots, \rho_{n} \in \Delta$,

$$
\rho:=\rho_{1} \oplus \rho_{2} \oplus \cdots \oplus \rho_{n} \oplus \bar{\rho}_{n} \oplus \cdots \oplus \bar{\rho}_{2} \oplus \bar{\rho}_{1}
$$

is a special object dominating $\rho_{1}, \rho_{2}, \ldots, \rho_{n}[2$; Theorem 3.4], i.e. $(\Delta, \hat{\varepsilon})$ is specially directed in the sense of [14; Sect. 5].

We are now in a position to establish the most novel aspect of Theorem 3.5, the existence of a complete normal field system with gauge symmetry by applying the spatial version of the cross product construction, [14; Corollary 6.2], to the $C^{*}$-algebra $\mathfrak{A}$, the permutation symmetric, specially directed semigroup $(\Delta, \hat{\varepsilon})$ of unital endomorphisms and the faithful representation $\pi_{0}$. This gives us a representation $\pi$ of $\mathfrak{A}$ on $\mathscr{H} \supset \mathscr{H}_{0}$, a compact group $G$ of unitaries on $\mathscr{H}$ and a homomorphism $\rho \rightarrow H_{\rho}$ from $\Delta$ to the semigroup of finite dimensional Hilbert spaces with support $I$ in $\mathscr{B}(\mathscr{H})^{20}$ such that
A) $\mathscr{H}_{0}=\{\Phi \in \mathscr{H}: g \Phi=\Phi, g \in G\}$,
B) $\pi_{0}$ is the restriction of $\pi$ to $\mathscr{H}_{0}$,
C) $\pi(\mathfrak{U}) \subset G^{\prime}$,
D) $\alpha_{g}\left(H_{\rho}\right):=g H_{\rho} g^{-1}=H_{\rho}, \quad \rho \in \Delta, \quad g \in G$,
E) $\psi \pi(A)=\pi \circ \rho(A) \psi, \quad \psi \in H_{\rho}, \quad A \in \mathfrak{A}, \quad \rho \in \Delta$,
F) $\vartheta\left(H_{\rho}, H_{\rho^{\prime}}\right)=\pi\left(\hat{\varepsilon}\left(\rho, \rho^{\prime}\right)\right), \quad \rho, \rho^{\prime} \in \Delta$,
G) $\left(u_{\rho}, u_{\rho^{\prime}}\right)=\pi\left(\left(\rho, \rho^{\prime}\right)\right), \quad \rho, \rho^{\prime} \in \Delta$,
H) the family $u_{\rho}, \rho \in \Delta$, separates the points of $G$,

where $\vartheta\left(H_{\rho}, H_{\rho^{\prime}}\right)$ denotes the canonical unitary operator permuting the factors in the tensor product of $H_{\rho}$ and $H_{\rho^{\prime}}$, i.e.

$$
\vartheta\left(H_{\rho}, H_{\rho^{\prime}}\right) \psi \psi^{\prime}=\psi^{\prime} \psi, \quad \psi \in H_{\rho}, \quad \psi^{\prime} \in H_{\rho^{\prime}},
$$

$u_{\rho}$ denotes the unitary representation of $G$ on $H_{\rho}$ induced by $\alpha_{g}$ on $H$ and $\left(u_{\rho}, u_{\rho^{\prime}}\right)$ denotes the space of interwiners between $u_{\rho}$ and $u_{\rho^{\prime}}$. Here the space of linear operators from $H_{\rho}$ to $H_{\rho^{\prime}}$, denoted $\left(H_{\rho}, H_{\rho^{\prime}}\right)$, is canonically identified with (the linear span of) $H_{\rho^{\prime}} \cdot H_{\rho}^{*}$ (cf. e.g. [23, Sect. 2]).

We now define $\mathfrak{F}(\mathcal{O})$ to be the von Neumann algebra generated by the $H_{\rho}$ as

\footnotetext{
${ }^{20}$ For an account of the properties of Hilbert spaces inside operator algebras the reader may consult [11; Sect. 3], [22; Sect. 2] or [23; Sect. 2]
} 
$\rho$ runs through $\Delta(\mathcal{O})$ and we must verify that $\{\pi, G, \mathfrak{F}\}$ is a complete, normal field system with gauge symmetry.

We let $\mathbf{U}(G)$ be the category of finite dimensional continuous unitary representations of $G$ with objects $u_{\rho}, \rho \in \Delta$, then F) and $\left.G\right)$ show that $T \rightarrow \pi(T)$ defines an isomorphism $^{21}$ from $(\mathscr{T}, \hat{\varepsilon})$ to $(\mathbf{U}(G), \vartheta)$. A standard application of the StoneWeierstraß Theorem using $\mathrm{H}$ ) (cf., for example, the proof of [23; Lemma 6.2]) shows that every $\xi \in \hat{G}$, the set of equivalence classes of irreducible, continuous, unitary representations of $G$, is realized one some $H_{\rho}$. This establishes, without appealing to the main theorem of [2], that the superselection structure of $\mathfrak{A}$ is described by the representation theory of a compact group $G$.

We next show that $\{\pi, G, \mathfrak{F}\}$ is a field system with gauge symmetry by verifying the conditions $\alpha), \beta, \gamma)$ and $\delta$ ) of Definition 3.1. It follows from $G)$ that $\pi\left(\left(\rho, \rho^{\prime}\right)\right) \subset \mathfrak{F}(\mathcal{O})$ whenever $\rho, \rho^{\prime} \in \Delta(\mathcal{O})$. In particular, any unitary of $\pi(\mathfrak{A}(\mathcal{O}))$ is in $\mathfrak{F}(\mathcal{O})$, thus $\pi(\mathfrak{A}(\mathcal{O}))$ is contained in the fixed-point algebra of $\mathfrak{F}(\mathcal{O})$ under $\alpha_{G}$. Harmonic analysis now shows that $\mathfrak{F}(\mathcal{O})$ is actually the weak closure of the span of the $H_{p}, \rho \in \Delta(\mathcal{O})$ (cf., for example, [11; Theorem 2.3]). Now if $\rho \in \Delta(\mathcal{O})$

$$
m\left(H_{\rho}\right)=m\left(\left(H_{\imath}, H_{\rho}\right)\right)=\left(u_{t}, u_{\rho}\right)=\pi((l, \rho)) \subset \pi(\mathfrak{U}(\mathcal{O}))
$$

and this verifies $\alpha$ ), $m$ denoting the mean over the action $\alpha_{G}$.

We pick for each $\xi \in \hat{G}$ a $\rho_{\xi} \in \Delta$ such that $u_{\rho}$ is of class $\xi$ then the $\pi_{\xi}:=\pi_{0} \circ \rho_{\xi}$ are mutually inequivalent irreducible representations so that by [14; Corollary 6.4] $\mathfrak{F}^{\prime}$ is isomorphic to $\pi_{0}(\mathfrak{A})^{\prime}=\mathbf{C I}$ verifying $\beta$ ).

Since each $\xi \in \hat{G}$ is realized on a Hilbert space in $\mathscr{F}(\mathcal{O})$ and, by $\mathrm{A}), \mathscr{H}_{0}$ is the space of $G$-invariant vectors, $\mathscr{H}_{0}$ is cyclic for $\mathscr{F}(\mathcal{O})$. The simple argument may be found in the proof of [14; Theorem 6.1]. This verifies $\gamma$ ). Finally $\delta$ ) is an immediate consequence of $\mathrm{E}$ ).

Our field system is complete since by [14; Proposition 6.3]

$$
\pi \cong \bigoplus_{\xi \in \hat{G}} d(\xi) \pi_{\xi}
$$

where $d(\xi)$ is the dimension of $\xi$, which by F) coincides with the statistical dimension of $\rho_{\xi}$. It only remains to show that our field system is normal. For each $\rho \in \Delta$, there is a unitary $\kappa(\rho) \in(\rho, \rho)$ with

$$
T \circ \kappa(\rho)=\kappa\left(\rho^{\prime}\right) \circ T, \quad T \in\left(\rho, \rho^{\prime}\right) .
$$

Such an element is uniquely determined by its values on irreducibles and $\kappa$ is fixed by requiring that $\kappa(\rho)= \pm 1_{\rho}$ according as $\rho$ is para-Bose or para-Fermi respectively. $\kappa$ is introduced in [21; Sect. 4] and is used in the definition of $\hat{\varepsilon}$. It is also discussed in [2; Sect. 7]. We have

$$
\kappa\left(\rho_{1}\right) \times \kappa\left(\rho_{2}\right)=\kappa\left(\rho_{1} \rho_{2}\right), \quad \rho_{1}, \rho_{2} \in \Delta .
$$

Now, as we have remarked, $\mathscr{T}$ and $\mathbf{U}(G)$ are isomorphic by $\mathbf{G})$. Hence (3.28) and

${ }^{21}$ More precisely, this is an isomorphism of strict symmetric monoidal $C^{*}$-categories. The terminology is explained in [2; Sect. 1] 
(3.29) imply that there is a unique $k \in G$ with $u_{\rho}(k)=\kappa(\rho), \rho \in \Delta^{22}$. Since $\kappa(\rho) \in(\rho, \rho)$, $k$ must be in the centre of $G$ and, since $\kappa(\rho){ }^{\circ} \kappa(\rho)=1, k^{2}=e$, the identity of $G$. Since $k$ is in the centre of $G$, it suffices to suppose in (3.2) that $F$ and $F^{\prime}$ transform irreducibly under $G$. In this case $F=B \psi$ and $F^{\prime}=B^{\prime} \psi^{\prime}$, where $B \in \pi\left(\mathfrak{U}\left(\mathcal{O}_{1}\right)\right)$, $B^{\prime} \in \pi\left(\mathfrak{A}\left(\mathcal{O}_{2}\right)\right), \psi \in H_{\rho}, \psi^{\prime} \in H_{\rho^{\prime}}$, with $\rho \in \Delta\left(\mathcal{O}_{1}\right)$ and $\rho^{\prime} \in \Delta\left(\mathcal{O}_{2}\right)$. Now

$$
\begin{aligned}
F F^{\prime} & =B \psi B^{\prime} \psi^{\prime}=B \rho\left(B^{\prime}\right) \psi \psi^{\prime}=B B^{\prime} \vartheta\left(H_{\rho^{\prime}}, H_{\rho}\right) \psi^{\prime} \psi \\
& =B^{\prime} B \pi\left(\hat{\varepsilon}\left(\rho^{\prime}, \rho\right)\right) \psi^{\prime} \psi= \pm B^{\prime} B \psi^{\prime} \psi= \pm B^{\prime} \psi^{\prime} B \psi= \pm F^{\prime} F,
\end{aligned}
$$

where the minus sign is to be taken if and only if both $\rho$ and $\rho^{\prime}$ are para-Fermi. But in this case

$$
\alpha_{k}(F)=B u_{\rho}(k) \psi=B \kappa(\rho) \psi=-B \psi=-F,
$$

and similarly $\alpha_{k}\left(F^{\prime}\right)=-F^{\prime}$, and we have verified (3.2) and completed the proof of existence in Theorem 3.5.

It is convenient to establish Theorem 3.6 before proving uniqueness in Theorem 3.5. In Theorem 3.6 we do not assume that we are dealing with a complete field system. This added degree of generality gives information on a situation of practical importance, where the theory has been defined using a field net and a gauge group, but the field net does not yet describe all sectors. We shall also take the opportunity of classifying all field systems with gauge symmetry up to unitary equivalence even though we believe that anomalous commutation relations have no practical importance.

We begin the proof of Theorem 3.6 with an elementary lemma.

3.8 Lemma. Let $\{\pi, G, \mathfrak{F}\}$ be a field system with gauge symmetry then $\mathscr{H}_{0}$ is the space of $G$-invariant vectors and is separating for $\mathfrak{F}$. Furthermore

$$
\begin{gathered}
\pi(\mathfrak{U})^{\prime}=G^{\prime \prime}, \\
\mathfrak{F}(\mathcal{O})^{\prime} \cap G^{\prime}=\pi\left(\mathfrak{U}\left(\mathcal{O}^{\prime}\right)\right)^{-}, \quad \mathcal{O} \in \mathscr{K} .
\end{gathered}
$$

Proof. By definition $G$ leaves $\mathscr{H}_{0}$ pointwise fixed and $\alpha$ ) with $\beta$ ) or $\gamma$ ) implies that any $G$-invariant vector lies in $\mathscr{H}_{0}$. Let $m$ denote the normal conditional expectation got by integrating $\alpha_{g}$ over the compact group $G$ using Haar measure. If $F \in \mathfrak{F}$ and $F \mathscr{H}_{0}=0$ then $m\left(F^{*} F\right) \mathscr{H}_{0}=0$ and since $m\left(F^{*} F\right) \in \pi(\mathfrak{A})$ and $\pi_{0}$ is faithful $m\left(F^{*} F\right)=0$. But $m$ itself is faithful so $F=0$ and $\mathscr{H}_{0}$ separates $\mathfrak{F}$. Now

$$
G^{\prime}=m(\mathscr{B}(\mathscr{H}))=m\left(\mathfrak{F}^{-}\right)=m(\mathfrak{F})^{-}=\pi(\mathfrak{A})^{-} .
$$

Here $^{-}$denotes the closure in the weak operator topology; see [10; Sect. III] for a detailed proof of these equalities. This proves (3.30). By $\alpha)$ and $\delta), \pi\left(\mathfrak{U}\left(\mathcal{O}^{\prime}\right)\right)^{-} \subset$ $\mathfrak{F}(\mathcal{O})^{\prime} \cap G^{\prime}$. Now by $\gamma$ ), an element $T$ of $\mathfrak{F}(\mathcal{O})^{\prime} \cap G^{\prime}$ is uniquely determined by its restriction to $\mathscr{H}_{0}$ which is an invariant subspace for $T$. Let $E_{0}$ be the projection onto $\mathscr{H}_{0}$ then $\mathfrak{F}(\mathcal{O})^{\prime} \cap G^{\prime} \subset \pi(\mathfrak{U}(\mathcal{O}))^{\prime}$ so

$$
T \mid \mathscr{H}_{0} \in \pi(\mathfrak{U}(\mathcal{O}))^{\prime} E_{0}=\pi_{0}(\mathfrak{A}(0))^{\prime}=\pi_{0}\left(\mathfrak{A}\left(\mathcal{O}^{\prime}\right)\right)^{-}
$$

by duality (3.1). This proves (3.31).

22 This is Tannaka's contribution [24] to the duality theory of compact groups which is discussed, for example, in the comments following Lemma 6.2 of [2] 
Let $\Xi$ denote the set of equivalence classes of irreducible subrepresentations of the defining representation of $G$ and if $\xi \in \Xi$, let $E_{\xi} \in G^{\prime} \cap G^{\prime \prime}=\pi(\mathfrak{A})^{-} \cap \pi(\mathfrak{H})^{\prime}$ be the corresponding central projection. Then (3.30) tells one that $\Xi$ is in 1-1-correspondence with the equivalence classes of irreducible subrepresentations of $\pi$ and that the class corresponding to $\xi \in \Xi$ has a multiplicity $d(\xi)$, the dimension of $\xi$.

It might seem that we are close to establishing c) of Theorem 3.6, but we have yet to establish that the representation $\pi_{\xi}$ corresponding to $\xi \in \Xi$ satisfies the selection criterion. This is our next task.

3.9 Lemma. Let $F_{1}, F_{2}, \ldots, F_{d} \in \mathfrak{F}(\mathcal{O})$ be a non-zero tensor transforming under $\alpha_{G}$ like a unitary representation, then if $\mathcal{O}^{-} \subset \mathcal{O}_{1}$ there is a tensor $X_{1}, X_{2}, \ldots, X_{d}$ in $\mathscr{F}\left(\mathcal{O}_{1}\right)$ transforming in the same way such that

$$
\sum_{i} X_{i}^{*} X_{i}=I \text {. }
$$

Proof. Regarding $F_{1}, F_{2}, \ldots, F_{d}$ as the entries of a column vector $F$, let $F=V|F|$ be the polar decomposition of $F$. Since the polar decomposition is unique, the entries of the partial isometry $V$ again transform in the same way under $\alpha_{G}$. But then $E=V^{*} V$ is a non-zero projection in $\mathfrak{U}(\mathcal{O})$ and, by Property B, there is an isometry $W \in \mathfrak{A}\left(\mathcal{O}_{1}\right)$ with $W W^{*}=E$. Taking $X=V W, X^{*} X=I$ so we may take $X_{1}, X_{2}, \ldots, X_{d}$ of the lemma to be the entries of $X$.

3.10 Lemma. If $\xi \in \Xi$, then $E_{\xi} \mathscr{H}$ is cyclic for $\mathfrak{F}(\mathcal{O})$.

Proof. Let $\mathcal{O}_{0}^{-} \subset \mathcal{O}$, then since $\mathscr{H}_{0}$ is cyclic for $\mathfrak{F}\left(\mathcal{O}_{0}\right)$, there is a non-zero tensor in $\mathfrak{F}\left(\mathcal{O}_{0}\right)$ transforming as a unitary representation of class $\xi$ and hence, by Lemma 3.9, a tensor $X_{1}, X_{2}, \ldots, X_{d} \in \mathfrak{F}(\mathcal{O})$ of class $\xi$ satisfying (3.32). But

$$
\mathfrak{F}(\mathcal{O}) \mathscr{H}_{0}=\mathfrak{F}(\mathcal{O}) \sum_{i} X_{i}^{*} X_{i} \mathscr{H}_{0} \subset \mathfrak{F}(\mathcal{O}) E_{\xi} \mathscr{H}
$$

so that $E_{\xi} \mathscr{H}$ is cyclic as required.

3.11 Lemma. Let $\{\pi, G, \mathfrak{F}\}$ be a field system with gauge symmetry and $\pi_{\xi}$ an irreducible subrepresentation of $\pi$ corresponding to $\xi \in \Xi$ then

$$
\pi_{\xi}\left|\mathfrak{A}\left(\mathcal{O}^{\prime}\right) \cong \pi_{0}\right| \mathfrak{A}\left(\mathcal{O}^{\prime}\right), \quad \mathcal{O} \in \mathscr{K} .
$$

Proof. By $\delta), \mathscr{F}(\mathcal{O}) \subset \pi\left(\mathfrak{H}\left(\mathcal{O}^{\prime}\right)\right)^{\prime}$ so by Lemma $3.10, E_{\xi} \mathscr{H}$ and $\mathscr{H}_{0}=E_{0} \mathscr{H}$ are cyclic subspaces for $\pi\left(\mathfrak{A}\left(\mathcal{O}^{\prime}\right)\right)^{\prime}$. Hence $E_{0}$ and $E_{\xi}$ have central supports $I$ in $\pi\left(\mathfrak{H}\left(\mathcal{O}^{\prime}\right)\right)^{\prime}$ so that we at least have quasiequivalence in (3.33). By Property B, the commutants of $\pi_{\xi} \mid \mathfrak{A}\left(\mathcal{O}^{\prime}\right)$ and $\pi_{0} \mid \mathfrak{H}\left(\mathcal{O}^{\prime}\right)$ are properly infinite so that unitary equivalence will follow once we know that the separability of $\mathscr{H}_{\pi_{\xi}}$ follows from that of $\mathscr{H}_{0}$. Since $\pi_{\xi}$ is irreducible, each non-zero vector $\Phi \in \mathscr{H}_{\pi_{\xi}}$ is cyclic and $\mathscr{H}_{\pi_{\xi}}$ is the closure of the union of $\pi_{\xi}\left(\mathfrak{H}\left(\mathcal{O}_{n}\right)\right) \Phi$ for an increasing sequence of double cones. Hence it suffices to show that $\pi_{\xi}(\mathfrak{H}(\mathcal{O})) \Phi$ is separable for each $\mathcal{O} \in \mathscr{K}$. Since $\mathscr{H}_{0}$ is separable, $\pi_{0}(\mathfrak{H}(\mathcal{O}))_{1}$ is compact metrizable in the weak operator topology. But we already know quasiequivalence in (3.33) for each $\mathcal{O} \in \mathscr{K}$ so $\pi_{\xi}(\mathfrak{H}(\mathcal{O}))_{1}$ is compact metrizable and $\pi_{\xi}(\mathfrak{U}(\mathcal{O})) \Phi$ is separable.

Remark. Lemma 3.11 is the only place where the separability of $\mathscr{H}_{0}$ is used. If $\mathscr{H}_{0}$ were not separable and if we were to impose (3.33) as an additional condition 
on a field system with gauge symmetry then Theorems 3.5 and 3.6 would remain valid. We shall shortly establish that $\pi_{\xi}$ has finite statistics.

We would like to establish that $\Xi=\hat{G}$. This was shown in [10; Theorem 3.6] using the cluster property. Here we follow a different route. We first show that if $\pi_{0} \circ \rho$ is an irreducible subrepresentation of $\pi$ with $\rho$ localized in $\mathcal{O}$ then there is a Hilbert space of support $I$ in $\mathscr{F}(\mathcal{O})$ inducing $\rho$. This was already established, under rather more restrictive hypotheses in [11].

If $\rho$ is irreducible then $\left(\pi_{0}{ }^{\circ} \rho, \pi\right)$, the space of intertwiners, is a Hilbert space with scalar product given by

$$
\left(V, V^{\prime}\right) 1_{\pi_{0} \circ \rho}=V^{*} V^{\prime} .
$$

If $\left(\pi_{0}{ }^{\circ} \rho, \pi\right) \neq 0$ and $\xi$ is the corresponding element of $\Xi$ then

$$
\sum_{i} V_{i} V_{i}^{*}=E_{\xi}
$$

where $V_{1}, V_{2}, \ldots, V_{d(\xi)}$ is an orthonormal basis of this Hilbert space. $\left(\pi_{0}{ }^{\circ} \rho, \pi\right)$ carries a unitary representation of $G$ of class $\xi$ given by the left multiplication with $g, g \in G$. Hence

$$
\sum_{i} V_{i}^{*} F V_{i}=\sum_{i} V_{i}^{*} \alpha_{g}(F) V_{i}=\sum_{i} V_{i}^{*} m(F) V_{i}, \quad F \in \mathscr{B}(\mathscr{H})
$$

We now prove a variant of [11; Proposition 2.1] which does not require that the vacuum vector be separating for each $\mathfrak{F}(\mathcal{O})$.

3.12 Lemma. Let $\{\pi, G, \mathfrak{F}\}$ be a field system with gauge symmetry and set

$$
H_{\rho}:=\{\psi \in \mathfrak{F}: \psi \pi(A)=\pi \circ \rho(A) \psi, A \in \mathfrak{X}\} .
$$

Then $H_{\rho}$ is a Hilbert space in $\mathfrak{F}$ and given $V \in\left(\pi_{0}^{\circ} \rho, \pi\right), \rho$ localized in $\mathcal{O}$ and irreducible, there is a unique $\psi \in \mathfrak{F}(\mathcal{O}) \cap H_{\rho}$ with

$$
\psi^{*} \Phi=V \Phi, \quad \Phi \in \mathscr{H}_{0} .
$$

Proof. $\mathscr{H}_{0}$ is separating for $\mathfrak{F}$ by Lemma 3.8. If $\psi, \psi^{\prime} \in H_{\rho}, \psi^{*} \psi^{\prime} \in \pi(\mathfrak{U})^{\prime} \cap \mathfrak{F}=G^{\prime \prime} \cap \mathfrak{F}$, hence $\psi^{*} \psi^{\prime} \Phi=\lambda \Phi, \Phi \in \mathscr{H}_{0}$ so $\psi^{*} \psi^{\prime}=\lambda I$ and $H_{\rho}$ is a Hilbert space in $\mathfrak{F}$ which might of course be $\{0\}$. However, given $V \in\left(\pi_{0}^{\circ} \rho, \pi\right), \psi$ is certainly uniquely specified by (3.37) and we have no option but to set

$$
\psi^{*} \sum_{k} F_{k} \Phi_{k}=\sum_{k} F_{k} V \Phi_{k}, \quad F_{k} \in \mathfrak{F}(\mathcal{O})^{\prime}, \quad \Phi_{k} \in \mathscr{H}_{0}
$$

We must, however, verify that (3.38) defines a bounded operator $\psi^{*}$. Now, using (3.35), we have

$$
\begin{aligned}
\left\|\sum_{k} F_{k} V \Phi_{k}\right\|^{2} & \leqq\|V\|^{2} \sum_{i}\left\|\sum_{k} F_{k} V_{i} \Phi_{k}\right\|^{2} \\
& =\|V\|^{2} \sum_{i, j, k}\left(\Phi_{j}, V_{i}^{*} m\left(F_{j}^{*} F_{k}\right) V_{i} \Phi_{k}\right) .
\end{aligned}
$$

By (3.31), $m\left(F_{j}^{*} F_{k}\right) \in \pi\left(\mathfrak{U}\left(\mathcal{O}^{\prime}\right)\right)^{-}$and, since $\rho$ is localized in $\mathcal{O}$,

$$
\left(\Phi_{j}, V_{i}^{*} m\left(F_{j}^{*} F_{k}\right) V_{i} \Phi_{k}\right)=\left(\Phi_{j}, m\left(F_{j}^{*} F_{k}\right) \Phi_{k}\right)=\left(\Phi_{j}, F_{j}^{*} F_{k} \Phi_{k}\right)
$$


Thus we conclude that

$$
\left\|\sum_{k} F_{k} V \Phi_{k}\right\|^{2} \leqq d(\xi)\|V\|^{2}\left\|\sum_{k} F_{k} \Phi_{k}\right\|^{2}
$$

so that $\psi^{*}$ is a well defined bounded operator, $\psi \in \mathfrak{F}(\mathcal{O})$ and (3.37) holds. Now

$$
\psi^{*} \pi \rho(A) \Phi=V \pi_{0}{ }^{\circ} \rho(A) \Phi=\pi(A) V \Phi=\pi(A) \psi^{*} \Phi, \quad \Phi \in \mathscr{H}_{0}, \quad A \in \mathfrak{A},
$$

and, since $\mathscr{H}_{0}$ is separating for $\mathfrak{F}$, we deduce that $\psi \in \mathscr{H}_{\rho}$ completing the proof of the lemma.

Using the above construction we have obtained a $d(\xi)$-dimensional Hilbert subspace of $H_{\rho}$. Let $\psi_{1}, \psi_{2}, \ldots, \psi_{d(\xi)}$ be an orthonormal basis of this subspace, then $\sum_{i} \psi_{i} \psi_{i}^{*} \in G^{\prime} \cap \mathfrak{F}(\mathcal{O})=\pi(\mathfrak{U}(\mathcal{O}))$. But (3.37) shows that $\sum_{i} \psi_{i} \psi_{i}^{*}$ is a multiple of the identity in restriction to $\mathscr{H}_{0}$. Thus $\sum_{i} \psi_{i} \psi_{1}^{*}=I$ and $H_{\rho}$ must be a $d(\xi)$-dimensional Hilbert space of support $I$. Taking $\rho=l$ as a particular case we have proved a) of Theorem 3.6. It also follows immediately from (3.36) that $\alpha_{g}\left(H_{\rho}\right)=H_{\rho}$ so that we have a continuous, unitary representation $u_{\rho}$ of $G$ induced on $H_{\rho}$.

3.13 Lemma. Let $\{\pi, G, \mathfrak{F}\}$ be a field system with gauge symmetry, then $\Xi=\hat{G}$ and for each $\xi \in \hat{G}$ there is an irreducible $\rho$ localized in $\mathcal{O}$ such that $u_{\rho}$ is of class $\xi$. Thus, in particular, the action of $G$ on $\mathfrak{F}(\mathcal{O})$ has full monoidal sepectrum.

Proof. Since $\mathscr{H}_{0}$ is cyclic and separating for $\mathscr{F}(\mathcal{O}), \Xi$ coincides with the spectrum of the action of $G$ on $\mathfrak{F}(\mathcal{O})$ and is hence automatically closed under conjugates. $\Xi$ separates the points of $G$ so that, if we can show that, given $\xi \in \Xi$, there is an irreducible $\rho$ localized in $\mathcal{O}$ such that $u_{\rho}$ is of class $\bar{\xi}$, then a standard application of the Stone-Weierstraß Theorem completes the proof. However, an irreducible subrepresentation $\pi_{\xi}$ of $\mathfrak{U}$ corresponding to $\xi \in \Xi$ must be equivalent to a representation of the form $\pi_{0}{ }^{\circ} \rho$ by Lemma 3.11 and $H_{\rho}$ constructed in Lemma 3.12 carries a representation of class $\bar{\xi}$, as required.

We now define a subset of localized morphisms:

$$
\Delta_{\mathcal{F}}:=\left\{\rho: H_{\rho} \text { is a finite dimensional Hilbert space of support } I\right\} .
$$

Note that $\Delta_{\mathfrak{F}}$ is trivially closed under products, direct sums and subobjects and we let $\mathscr{T}_{\mathscr{F}}$ denote the category with objects $\Delta_{\mathfrak{F}}$ and with $\left(\rho, \rho^{\prime}\right)$ as the set of arrows between $\rho$ and $\rho^{\prime}$. Let $\mathbf{U}(G)$ denote the category of representations with objects $u_{\rho}, \rho \in \Delta_{\mathfrak{F}}$.

3.14 Theorem. Let $\{\pi, G, \mathfrak{F}\}$ be a field system with gauge symmetry, then the map $T \in\left(\rho, \rho^{\prime}\right) \rightarrow \pi(T) \in\left(H_{\rho}, H_{\rho^{\prime}}\right)$ defines an isomorphism ${ }^{23}$ from $\mathscr{T}_{\mathfrak{F}}$ to $\mathbf{U}(G)$. Every equivalence class of finite dimensional, continuous, unitary representations of $G$ is realized on some $H_{\rho}, \rho \in \Delta_{\mathfrak{F}} . \Delta_{\mathfrak{F}} \subset \Delta$ and is closed under conjugates. The statistical dimension of $\rho$ equals the dimension of $u_{\rho}$. The irreducibles of $\Delta_{\mathfrak{F}}$ are precisely the irreducibles $\rho \in \Delta$ for which $\left(\pi_{0}^{\circ} \rho, \pi\right) \neq 0$. The span of $\left\{H_{\rho}: \rho \in \Delta_{\mathfrak{F}}(\mathcal{O}), \rho\right.$ irreducible $\}$ is a weakly dense subspace of $\mathfrak{F}(\mathcal{O})$.

${ }^{23}$ More precisely an isomorphism of strict monoidal $C^{*}$-categories, cf. [2; Sect. 1] 
Proof. Since, by definition, $\pi(\mathfrak{A})$ is the fixed-point algebra of the action of $G$ on $\mathfrak{F}$, the isomorphism of $\mathscr{T}_{\mathfrak{F}}$ and $\mathbf{U}(G)$ is a trivial consequence of $\pi(\mathfrak{Q})^{\prime} \cap \mathfrak{F}=\mathbf{C I}$ (cf. [23; Lemma 2.4] or [11; Theorem 3.6]). It is now clear that every finite dimensional, continuous, unitary representation of $G$ is realized on some $H_{\rho}, \rho \in \Delta_{\mathfrak{f}}$ and that each object $\rho \in \Delta_{\mathfrak{F}}$ is a finite direct sum of irreducibles. It was, in effect, shown in [11; Theorem 4.1] that each irreducible $\rho \in \Delta_{\mathfrak{F}}$ obeys parastatistics whose order is the dimension of $u_{\rho}$. Hence the statistical dimension of $\rho$ equals the dimension of $u_{\rho}$ for all $\rho \in \Delta_{\mathfrak{F}}$, and thus $\Delta_{\mathfrak{F}} \subset \Delta$. The notion of conjugation in $\Delta_{\mathfrak{f}}$ must coincide with that in $\mathbf{U}(G)$, since, for irreducibles, a conjugate $\bar{\rho}$ for $\rho$ is defined up to equivalence by requiring $\bar{\rho}$ to be irreducible and $(l, \bar{\rho} \rho) \neq 0$. Hence $\Delta_{\mathfrak{F}}$ is closed under conjugates. If $\left(\pi_{0}^{\circ} \rho, \pi\right)=0$, then $H_{\rho}=0$ since $\mathscr{H}_{0}$ is separating for $\mathfrak{F}$. Hence the irreducibles of $\Delta_{\mathfrak{F}}$ are precisely the irreducibles $\rho \in \Delta$ for which $\left(\pi_{0}^{\circ} \rho, \pi\right) \neq 0$. Finally, it is a simple matter of harmonic analysis to see that the span of $\left\{H_{\rho}: \rho \in \Delta_{\mathfrak{F}}(\mathcal{O}), \rho\right.$ irreducible $\}$ is a weakly dense subspace of $\mathfrak{F}(\mathcal{O})$ since we know that each $\xi \in \hat{G}$ is realized on some such $H_{\rho}$, cf. [11; Theorem 2.3].

Remark. Every finite dimensional $\alpha_{G}$-invariant Hilbert space in $\mathbb{F}(\mathcal{O})$ is of the form $H_{\rho}$, for some $\rho \in \Delta_{\mathfrak{f}}(\mathcal{O})$.

In the course of proving Theorem 3.14 we have also completed the proof of $c$ ) of Theorem 3.6. Now let $\gamma$ be an automorphism of $\mathfrak{F}$ leaving $\pi(\mathfrak{A})$ pointwise fixed, then $\gamma\left(H_{\rho}\right)=H_{\rho}$ so that we have a unitary $v_{\rho}$ defined on $H_{\rho}$ which must satisfy $v_{\rho \rho^{\prime}}=v_{\rho} \rho\left(v_{\rho^{\prime}}\right)=v_{\rho} \otimes v_{\rho^{\prime}}$. Hence by Tannaka's result [24], there is a unique $g \in G$ with $v_{\rho}=u_{\rho}(g)$, i.e. $\alpha_{g}(\psi)=\gamma(\psi), \psi \in H_{\rho}, \rho \in \Delta_{\mathfrak{g}}$. Since, as we have just seen, the $H_{\rho}$ generate $\mathfrak{F}$, we conclude that $\gamma=\alpha_{g}$ proving b) of Theorem 3.6. The remaining points still to be proved in Theorem 3.6 concern normal field systems.

If $\{\pi, G, \mathfrak{F}\}$ is normal and $\Phi \in \mathscr{H}_{\xi}$, the Hilbert space of $\pi_{\xi}$, then, since $k$ is in the centre of $G$ and $k^{2}=e$, we anyway have $k \Phi= \pm \Phi$. Writing $\pi_{\xi} \cong \pi_{0} \circ \rho$ and taking $\psi \in H_{\rho}$ we then have $\alpha_{k}(\psi)= \pm \psi$. We now compute $\varepsilon(\rho, \rho)$. Let $\rho^{\prime}$ be equivalent to $\rho$ and have a spacelike separated support, then $H_{\rho}$ and $H_{\rho^{\prime}}$ transform according to equivalent (irreducible) representations of $G$ and we have $\sum_{i} \psi_{i}^{\prime} \psi_{i}^{*}=\pi(V)$ with $V \in\left(\rho, \rho^{\prime}\right)$ unitary, where $\psi_{1}, \psi_{2}, \ldots, \psi_{d}$ and $\psi_{1}^{\prime}, \psi_{2}^{\prime}, \ldots, \psi_{d}^{\prime}$ are orthonormal bases of $H_{\rho}$ and $H_{\rho^{\prime}}$ transforming in the same way under $G$. Since $\varepsilon\left(\rho, \rho^{\prime}\right)=1_{\rho \rho^{\prime}}, \varepsilon(\rho, \rho)=$ $V^{*} \times 1_{\rho}^{\circ} 1_{\rho} \times V$ by the analogue of (3.15). Hence

$$
\pi(\varepsilon(\rho, \rho))=\sum_{i, j, k} \psi_{i} \psi_{i}^{\prime *} \psi_{j} \psi_{k}^{\prime} \psi_{k}^{*} \psi_{j}^{*}= \pm \sum_{i, j} \psi_{i} \psi_{j} \psi_{i}^{*} \psi_{j}^{*}= \pm \vartheta\left(H_{\rho}, H_{\rho}\right)
$$

so that the plus and minus signs correspond to para-Bose and para-Fermi statistics respectively. This proves d) of Theorem 3.6.

The computation we have just made also shows that

$$
\vartheta\left(H_{\rho}, H_{\rho^{\prime}}\right)=\pi\left(\hat{\varepsilon}\left(\rho, \rho^{\prime}\right)\right), \quad \rho, \rho^{\prime} \in \Delta_{\mathfrak{F}}
$$

for, by virtue of (3.15) and the analogous property of $\vartheta\left(H_{\rho}, H_{\rho^{\prime}}\right)$, it suffices to take $\rho$ and $\rho^{\prime}$ to be spacelike separated irreducibles. But, in this case, since the field system is normal

$$
\vartheta\left(H_{\rho}, H_{\rho^{\prime}}\right) \psi \psi^{\prime}=\psi^{\prime} \psi= \pm \psi \psi^{\prime}, \quad \psi \in H_{\rho}, \quad \psi^{\prime} \in H_{\rho^{\prime}},
$$


where the minus sign is to be taken if and only if $\alpha_{k}(\psi)=-\psi$ and $\alpha_{k}\left(\psi^{\prime}\right)=-\psi^{\prime}$. Thus, by the above computation, $\vartheta\left(H_{\rho}, H_{\rho^{\prime}}\right)= \pm 1_{H_{\rho} H_{\rho^{\prime}}}$, where the minus sign holds if and only if both $\rho$ and $\rho^{\prime}$ are para-Fermi, and, in view of (3.20), we have established (3.40).

To complete the proof of Theorem 3.6, we must show that a normal field system satisfies twisted duality. We first remark that if $V$ is given by (3.8) then $V F V^{*}=F_{+}+i k F_{-}$and that since $V \in \pi(\mathfrak{H})^{\prime} \cap G^{\prime},\left\{\pi, G, \mathfrak{F}^{t}\right\}$ will also be a normal field system with gauge symmetry. Now let $F \in \mathscr{F}^{t}(\mathcal{O})^{\prime}$ transform irreducibly under

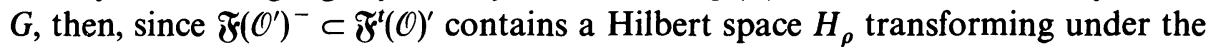
same equivalence class of irreducible representations by Lemma 3.13, $F=B \psi$ with

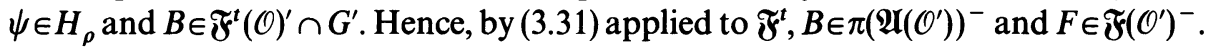
Thus $\mathfrak{F}\left(\mathcal{O}^{\prime}\right)^{-}=\mathfrak{F}^{t}(\mathcal{O})^{\prime}$ and taking commutants we get (3.9).

Now consider a complete normal field system with gauge symmetry, $\{\pi, G, \mathfrak{F}\}$, then $\Delta_{\mathfrak{r}}=\Delta$ and, in the course of proving Theorem 3.6, we have established the existence of a homomorphism $\rho \rightarrow H_{\rho}$ from $\Delta$ to the semigroup of Hilbert spaces of support $I$ in $B(\mathscr{H})$ satisfying $\mathrm{A})-\mathrm{H})$. But, by [14;Corollary 6.2] a system $\left(\mathscr{H}, \pi, G, \rho \in \Delta \rightarrow H_{\rho}\right)$ is unique up to unitary equivalence. However, $\mathscr{F}(\mathcal{O})$ is generated by $\left\{H_{\rho}: \rho \in \Delta(\mathcal{O})\right\}$ so any two complete normal field systems are equivalent in the sense of Definition 3.4 and we have completed the proof of Theorem 3.5.

We can at this point classify field systems with gauge symmetry up to equivalence. In place of (3.40), we exploit the isomorphism of $\mathscr{T}_{\mathfrak{F}}$ and $\mathbf{U}(G)$ to define a (permutation) symmetry $\varepsilon_{\mathfrak{z}}$ by

$$
\vartheta\left(H_{\rho}, H_{\rho^{\prime}}\right)=\pi\left(\varepsilon_{\mathfrak{F}}\left(\rho, \rho^{\prime}\right)\right), \quad \rho, \rho^{\prime} \in \Delta_{\mathfrak{F}} .
$$

Thus the analogues of (3.15)-(3.18) hold and $(\mathbf{U}(G), \vartheta)$ and $\left(\mathscr{T}_{\mathfrak{F}, \text {, }}\right)$ are isomorphic by construction. In particular, $\left(\Delta_{\mathfrak{F}}, \varepsilon_{\mathfrak{g}}\right)$ will be a permutation symmetric semigroup which is specially directed in the sense of [14; Sect. 5] (cf. Lemma 3.7). Appealing to the uniqueness result of $[14$; Corollary 6.2$]$ we see that $\{\pi, G, \mathfrak{F}\}$ is determined up to equivalence by the pair $\left(\Delta_{\mathfrak{g}}, \varepsilon_{\mathfrak{f}}\right)$. Conversely, given any pair $\left(\Delta^{\prime}, \varepsilon^{\prime}\right)$ consisting of a subsemigroup $\Delta^{\prime}$ of $\Delta$ and symmetry $\varepsilon^{\prime}$ for the full subcategory $\mathscr{T}^{\prime}$ of $\mathscr{T}$ determined by $\Delta^{\prime}$ making $\left(\mathscr{T}^{\prime}, \varepsilon^{\prime}\right)$ into a strict symmetric monoidal $C^{*}$-category with conjugates ${ }^{24}$ in the sense of $[2$; Sect. 1$],\left(\Delta^{\prime}, \varepsilon^{\prime}\right)$ will be a permutation symmetric semigroup which is specially directed in the sense of [14; Sect. 5] and we may construct, as in the proof of existence in Theorem 3.5, a field system with gauge symmetry $\{\pi, G, \mathfrak{F}\}$ for which $\Delta_{\mathfrak{F}}=\Delta^{\prime}$ and $\varepsilon_{\mathfrak{F}}=\varepsilon^{\prime}$. Hence we have

3.15 Theorem. Let $\{\pi, G, \mathfrak{F}\}$ be a field system with gauge symmetry then the map $\{\pi, G, \mathfrak{F}\} \rightarrow\left(\Delta_{\mathfrak{z}}, \varepsilon_{\mathfrak{f}}\right)$ determines a 1-1 correspondence between equivalence classes of field systems and pairs consisting of a subsemigroup of $\Delta$ closed under direct sums, subobjects and conjugates and a symmetry making the corresponding full subcategory of $\mathscr{T}$ into a strict symmetric monoidal $C^{*}$-category with conjugates (cf. [2; Sect. 1]).

The following simple construction will help to clarify the relationship between the different nets.

${ }^{24}$ The notion of conjugate is easily seen to be independent of the choice of symmetry 
3.16 Proposition. Let $\{\pi, G, \mathfrak{F}\}$ be a field system with gauge symmetry and $N$ a closed normal subgroup of $G$ then we can construct another such system $\left\{\pi^{N}, G / N, \mathfrak{F}^{N}\right\}$ as follows: let $\pi^{N}$ denote the restriction of $\pi$ to the invariant subspace

$$
\mathscr{H}^{N}=\{\Phi \in \mathscr{H}: g \Phi=\Phi, g \in N\} .
$$

The restriction of $G$ to the invariant subspace is naturally isomorphic to $G / N$. Let $\mathfrak{F}^{N}$ be the restriction of the $\alpha_{N}$-invariant subnet of $\mathfrak{F}$ to the invariant subspace $\mathscr{H}^{N}$. Furthermore,

$$
\Delta_{\mathfrak{F}^{N}}=\left\{\rho \in \Delta_{\mathfrak{F}}: u_{\rho}(g)=1_{H_{\rho}}, g \in N\right\}
$$

and $\varepsilon_{\mathfrak{F}^{N}}$ is the restriction of $\varepsilon_{\mathfrak{F}}$ to $\Delta_{\mathfrak{F}^{N}}$.

Proof. $\mathscr{H}_{0}$ is a subspace of $\mathscr{H}^{N}$ so $\pi_{0}$ is still a subrepresentation of $\pi^{N}$ and $H^{N}$ is then trivially separating for $₹$. Hence the restriction map of the $\alpha_{N}$-invariant subnet of $\mathfrak{F}$ onto $\mathscr{F}^{N}$ is an isomorphism. By definition, the action of $G$ on the $\alpha_{N}$-invariant subnet of $\mathscr{F}$ factors through $N$ and $G / N$ acts faithfully on this subnet since the representations $u_{\rho}$ with $u_{\rho}(g)=1_{H_{\rho}}, g \in N$, separate the points of $G / N$. Indeed, each equivalence class of irreducible representations appears in this way. Thus $G / N$ acts faithfully on $\mathfrak{F}^{N}$ and the fixed-point net is trivially $\pi^{N}(\mathfrak{a})$. Furthermore, it is now clear that $N$ is the kernel of the restriction mapping of $G$ onto $\mathscr{H}^{N}$. Hence we have verified $\alpha$ ). If $m^{N}$ denotes the normal conditional expectation got by integrating over $N$ with respect to Haar measure then

$$
m^{N}(\mathscr{B}(\mathscr{H}))=m^{N}\left(\mathfrak{F}^{-}\right)=m^{N}(\mathfrak{F})^{-} .
$$

Reducing to the subspace $\mathscr{H}^{N}$ we see that $\mathfrak{F}^{N}$ is irreducible verifying $\beta$ ). Given $\Phi \in \mathscr{H}^{N}$, there is a sequence $\Phi_{k} \in \mathfrak{F}(\mathcal{O}) \mathscr{H}_{0}$ which also tends to $\Phi$. Hence $\mathscr{H}_{0}$ is cyclic for $\mathscr{F}^{N}(\mathcal{O})$ verifying $\left.\gamma\right)$. If $F \in m^{N}(\mathfrak{F}(\mathcal{O}))$ and $A \in \mathfrak{U}\left(\mathcal{O}^{\prime}\right)$ then $F \pi(A)=\pi(A) F$ and, restricting to the invariant subspace, we see that $\pi^{N}\left(\mathfrak{U}\left(\mathcal{O}^{\prime}\right)\right) \subset \mathfrak{F}^{N}(\mathcal{O})^{\prime}$. Since $\mathscr{H}^{N}$ is separating for the $\alpha_{N}$-invariant part of $\mathfrak{F}$, the subspace $H_{\rho} \subset \mathfrak{F}^{N}$ for $\rho \in \Delta_{\mathfrak{F}^{N}}$ is just the restriction of the corresponding subspace of $\mathfrak{F}$. Hence, bearing in mind the definition of $\mathfrak{F}^{N}$,

$$
\Delta_{\mathfrak{F}^{N}} \subset\left\{\rho \in \Delta_{\mathfrak{\gamma}}: u_{\rho}(g)=1_{H_{\rho}}, g \in N\right\} .
$$

On the other hand, we must have equality here since if $u_{\rho}(g)=1_{H_{\rho}}$, then $H_{\rho}$ is $\alpha_{N}$-invariant and its restriction to $\mathscr{H}^{N}$ will be a finite-dimensional Hilbert space of support $I$ in $\mathfrak{F}^{N}, \varepsilon_{\mathfrak{F}^{N}}$ is trivially the restriction of $\varepsilon_{\mathfrak{F}}$ to $\Delta_{\mathfrak{F}^{N}}$ completing the proof of the proposition.

The field system $\left\{\pi^{N}, G / N, \mathfrak{F}^{N}\right\}$ is a subsystem of $\{\pi, G, \mathfrak{F}\}$ and we show that all subsystems arise in this way.

3.17 Proposition. Given field systems with gauge symmetry $\left\{\pi_{1}, G_{1}, \mathfrak{F}_{1}\right\}$ and $\{\pi, G, \mathfrak{F}\}$ and an isometry $V: \mathscr{H}_{1} \rightarrow \mathscr{H}$ such that

$$
\begin{aligned}
V \pi_{1}(A) & =\pi(A) V, \quad A \in \mathfrak{A}, \\
V G_{1} V^{*} & =G E, \\
V \mathfrak{F}_{1} V^{*} & =\left(\mathfrak{F} \cap\{E\}^{\prime}\right) E,
\end{aligned}
$$


where $E=V V^{*}$ then there is a closed normal subgroup $N$ of $G$ such that $E$ is the projection onto the subspace of $N$-invariant vectors and $\left\{\pi_{1}, G_{1}, \mathfrak{F}_{1}\right\}$ is equivalent to $\left\{\pi^{N}, G / N, \mathfrak{F}^{N}\right\}$.

Proof. Equation (3.44) implies $E \in \pi(\mathfrak{U})^{\prime}$ and $E_{0} \leqq E$. In particular, $E$ is separating for $\mathfrak{F}$. Let $H_{1 \rho}$ be the Hilbert space in $\mathfrak{F}_{1}$ corresponding to $\rho \in \Delta_{\mathfrak{F}_{1}}$ then given $\psi_{1} \in H_{1 \rho}$, there exists a unique $\psi \in \mathfrak{F}$ with $V \psi_{1} V^{*}=\psi E$. Since $\psi_{1} \pi_{1}(A)=\pi_{1}^{\circ} \rho(A) \psi_{1}$, we get $\psi \pi(A) E=\pi \circ \rho(A) \psi E$, and hence $\psi \in H_{\rho}$. Since $H_{1 \rho}$ has support $I, H_{\rho}$ must have support $I$ so $\Delta_{\mathfrak{F}_{1}} \subset \Delta$. Since $\Delta_{\mathfrak{F}_{1}}$ is closed under products, direct sums, subobjects and conjugates, there is a closed normal subgroup $N$ of $G$ such that

$$
\Delta_{\mathfrak{F}_{1}}=\left\{\rho \in \Delta: u_{\rho}(g)=1_{H_{\rho}}, g \in N\right\}=\Delta_{\mathfrak{F}^{N}} .
$$

By (3.44), $\varepsilon_{\mathfrak{F}_{1}}=\varepsilon_{\mathfrak{F}^{N}}$ so by Proposition $3.16\left\{\pi_{1}, G_{1}, \mathfrak{F}_{1}\right\}$ and $\left\{\pi^{N}, G / N, \mathfrak{F}^{N}\right\}$ are isomorphic. Now $E \mathscr{H}=V \mathfrak{F}_{1} \mathscr{H}_{0}=V \mathscr{F}_{1} V^{*} \mathscr{H}_{0}=\mathscr{F}^{N} \mathscr{H}_{0}=\mathscr{H}^{N}$ so that $E$ is just the projection onto the subspace of $N$-invariant vectors as required.

We recall that a net $\mathfrak{F}$ over $\mathscr{K}$ is said to be additive if

$$
\mathcal{O}=\bigcup_{i} \mathcal{O}_{i} \text { implies } \mathfrak{F}(\mathcal{O})=\bigvee_{i} \mathfrak{F}\left(\mathcal{O}_{i}\right),
$$

the von Neumann algebra generated by the $\mathfrak{F}\left(\mathcal{O}_{i}\right)$.

3.18 Proposition. Let $\{\pi, G, \mathfrak{F}\}$ be a field system with gauge symmetry then $\mathfrak{F}$ is additive if and only if $\mathfrak{A}$ is additive.

Proof. Let $\rho \in \Delta_{\mathfrak{F}}(\mathcal{O})$, then there is a unitary $U \in\left(\rho^{\prime}, \rho\right) \subset \mathfrak{U}(\mathcal{O})$ with $\rho^{\prime} \in \Delta_{\mathfrak{F}}\left(\mathcal{O}_{1}\right)$. Hence $H_{\rho}=\pi(U) H_{\rho^{\prime}}$. If $\mathfrak{A}$ satisfies additivity then $H_{\rho}=\pi(U) H_{\rho^{\prime}} \subset \bigvee_{i} \mathfrak{F}\left(\mathcal{O}_{i}\right)$ so $\mathfrak{F}(\mathcal{O})=$ $\bigvee \mathfrak{F}\left(\mathcal{O}_{i}\right)$. The converse involves more work and we refer to [25; Theorem 1.3] for $i$

details remarking that we are here dealing exclusively with double cones which are connected.

\section{Superselection Structure for Topological Charges}

We come now to the analysis of topological charge. The goal of this section is to start from representations satisfying (1.2) and give a selfcontained account of superselection structure: composition of charges, existence of statistics and charge conjugation. These results then allow us to associate a compact group dual with the superselection structure (cf. Theorem 4.20 and the subsequent comments). They will be used in the next section to construct a field net over spacelike cones acted on by a gauge group.

We again draw the reader's attention to the role of the dimension of spacetime in this discussion. Whilst the analysis of statistics for localizable charges is valid for two or more space dimensions, the basic properties of the intertwiner calculus for topological charges, in particular Lemma 4.2 below, can only be established in three or more space dimensions.

Thus this section extends the results of $[1,9]$ to the set of representations satisfying (1.2). This task was first carried out by Buchholz and Fredenhagen 
$[6]^{25}$. As their discussion is relatively technical, we have thought it worthwhile to present in this section a simpler approach which is also conceptually clearer as it avoids having to introduce an auxiliary cone and an associated $C^{*}$-algebra.

Our discussion here will depend on the duality relation ${ }^{26}$

$$
\pi_{0}\left(\mathfrak{U}\left(\mathscr{C}^{\prime}\right)\right)^{\prime}=\pi_{0}(\mathfrak{U}(\mathscr{C}))^{-}, \quad \mathscr{C} \in \mathscr{J} .
$$

and the analogue of Property B.

Property $\mathbf{B}^{\prime}$. If $E \in \mathfrak{U}\left(\mathscr{C}^{\prime}\right)^{\prime}$ is a non-zero projection then, for any spacelike cone $\mathscr{C}_{1}$ containing the closure of $\mathscr{C}$ there is an isometry $W \in \mathfrak{U}\left(\mathscr{C}_{1}^{\prime}\right)^{\prime}$ with $W W^{*}=E$.

This property is again a consequence of standard structural assumptions of Quantum Field Theory, viz. covariance, positivity of the energy and locality. We denote by $(S)$ the set of representations satisfying the selection criterion (1.2). It follows from Property $B^{\prime}$ that $(S)$ is closed under direct sums and subrepresentations.

If $\pi \in(S)$ and we pick a unitary $V$ such that

$$
V \pi(A)=\pi_{0}(A) V, \quad A \in \mathfrak{U}\left(\mathscr{C}^{\prime}\right)
$$

and set

$$
\rho(A)=V \pi(A) V^{*}, \quad A \in \mathfrak{A},
$$

then we get an equivalent representation defined on $\mathscr{H}_{0}$ which has the property of acting trivially on $\mathfrak{U}\left(\mathscr{C}^{\prime}\right)$,

$$
\rho(A)=\pi_{0}(A), \quad A \in \mathfrak{U}\left(\mathscr{C}^{\prime}\right) .
$$

If $\mathscr{C}_{1} \supset \mathscr{C}$, then $\rho\left(\mathfrak{U}\left(\mathscr{C}_{1}\right)\right) \subset \pi_{0}\left(\mathfrak{\mathcal { U }}\left(\mathscr{C}_{1}^{\prime}\right)\right)^{\prime}=\pi_{0}\left(\mathfrak{A}\left(\mathscr{C}_{1}\right)\right)^{-}$, but it does not follow that $\rho$ defines an endomorphism of $\mathfrak{U}$ because $\pi_{0}(\mathfrak{U})$ does not contain the von Neumann algebra $\pi_{0}\left(\mathfrak{A}\left(\mathscr{C}_{1}\right)\right)^{-}$. The representations $\rho$ are the analogues of our localized morphisms and we write $\Delta_{t}(\mathscr{C})$ to denote the set of representations satisfying (1.2) and (4.3). Although we cannot use the composition laws for endomorphisms, we show that if $\pi \in(S)$ and $\rho \in \Delta_{t}(\mathscr{C})$ then $\pi \rho$ can nevertheless be defined consistently.

4.1 Lemma. If $\pi \in(S)$ and $\rho \in \Delta_{t}(\mathscr{C})$ then there is a unique representation $\pi \rho$ of $\mathfrak{A}$ such that if $A \in \mathfrak{A}(\mathcal{O})$ and $V_{1} \in\left(\rho_{1}, \pi\right)$ is unitary, where $\rho_{1} \in \Delta_{t}\left(\mathscr{C}_{1}\right)$ and $\mathscr{C}_{1} \subset \mathscr{C}^{\prime} \cap \mathcal{O}^{\prime}$ then

$$
\pi \rho(A)=V_{1} \rho(A) V_{1}^{*} .
$$

25 Actually Buchholz and Fredenhagen study representations satisfying •

$$
\pi\left|\mathfrak{U}^{c}(\mathscr{C}) \simeq \pi_{0}\right| \mathfrak{U}^{c}(\mathscr{C}), \quad \mathscr{C} \in \mathscr{J}
$$

where $\mathfrak{A}^{c}(\mathscr{C}):=\{A \in \mathfrak{U}: A B=B A, B \in \mathfrak{A}(\mathscr{C})\}$, the relative commutant of $\mathfrak{Q}(\mathscr{C})$. They demonstrate that this stronger condition is automatically satisfied in a translation-covariant representation where the lower boundary of the energy-momentum spectrum is an isolated mass hyperboloid, i.e. in a massive 1-particle representation. In contrast to the case of localizable charges, this stronger condition is automatically equivalent to (1.2) as a consequence of duality (4.1). We prefer to use the form (1.2) which parallels our discussion of localizable charges.

26 Buchholz and Fredenhagen, of course, need only the corresponding weaker duality assumption $\pi_{0}\left(\mathfrak{A}^{c}(\mathscr{C})\right)^{\prime}=\pi_{0}(\mathfrak{A}(\mathscr{C}))^{-}$. This condition may well prove to be equivalent to (4.1) in practice 
Remark. The idea behind this definition is that, under the stated support condition $\rho_{1}$ should act trivially on $\rho(A)$ and hence (4.4) should define $\pi \rho(A)$ correctly.

Proof. Provided we can show that (4.4) defines $\pi \rho(A)$ unambiguously, we shall have a unique representation $\pi \rho$. To see that $\pi \rho(A)$ is independent of the choice of $V_{1}$ note first that if $B \in \mathfrak{U}\left(\mathscr{C}_{1}\right)$ then ${ }^{27}$

$$
\rho(A) B=\rho(A B)=\rho(B A)=B \rho(A),
$$

thus $\rho(A) \in \mathfrak{U}\left(\mathscr{C}_{1}\right)^{\prime}=\mathfrak{A}\left(\mathscr{C}_{1}^{\prime}\right)^{-}$. Given a unitary $V_{2} \in\left(\rho_{2}, \pi\right)$, where $\rho_{2} \in \Delta_{t}\left(\mathscr{C}_{2}\right)$ and $\mathscr{C}_{2} \subset \mathscr{C}^{\prime} \cap \mathcal{O}^{\prime}$, suppose first that $\mathscr{C}_{2} \subset \mathscr{C}_{1}$ and pick $C \in \mathfrak{A}\left(\mathscr{C}_{1}^{\prime}\right) \subset \mathfrak{U}\left(\mathscr{C}_{2}^{\prime}\right)$, then

$$
V_{2}^{*} V_{1} C=V_{2}^{*} V_{1} \rho_{1}(C)=\rho_{2}(C) V_{2}^{*} V_{1}=C V_{2}^{*} V_{1},
$$

thus $V_{1} \rho(A) V_{1}^{*}=V_{2} \rho(A) V_{2}^{*}$. However, by Lemmas A.7 and A.8, $\mathscr{C}^{\prime}$ is spacelike cone connected (cf. Definition A.6) and so, by Lemma A.5, is $\mathscr{C}^{\prime} \cap \mathcal{O}^{\prime}$, thus $V_{1} \rho(A) V_{1}^{*}=V_{2} \rho(A) V_{2}^{*}$ for any $\mathscr{C}_{2} \subset \mathscr{C}^{\prime} \cap \mathcal{O}^{\prime}$. We still need to show that $\pi \rho(A)$ does not depend on the choice of localization regions for $\rho$ and $A$. Suppose $\rho \in \Delta_{t}(\widehat{\mathscr{C}})$ and $A \in \mathfrak{U}(\hat{\mathcal{O}})$ then, since $\hat{\mathscr{C}}^{\prime} \cap \mathscr{C}^{\prime} \cap \mathcal{O}^{\prime} \cap \hat{\mathcal{O}}^{\prime}$ still contains a spacelike cone by Lemma A.5 and e) of the Appendix, (4.4) determines $\pi \rho(A)$ unambiguously as required.

We shall see later that $\pi \rho \in(S)$. In the next lemma we take a first step towards establishing the commutation properties of intertwiners.

4.2 Lemma. Let $T_{1} \in\left(\rho_{1}, \rho_{3}\right)$ and $T_{2} \in\left(\rho_{2}, \rho_{4}\right)$ with $\rho_{i} \in \Delta_{t}\left(\mathscr{C}_{i}\right)$ and $\mathscr{C}_{2} \cup \mathscr{C}_{4} \subset \mathscr{C}_{1}^{\prime} \cap \mathscr{C}_{3}^{\prime}$, then $T_{1} T_{2}=T_{2} T_{1}$.

Proof. Suppose first that there is a spacelike cone $\mathscr{C}_{6} \supset \mathscr{C}_{2} \cup \mathscr{C}_{4}$ with $\mathscr{C}_{6} \subset \mathscr{C}_{1}^{\prime} \cap \mathscr{C}_{3}^{\prime}$. Then if $A \in \mathfrak{A}\left(\mathscr{C}_{6}^{\prime}\right)$ we have

$$
T_{2} A=T_{2} \rho_{2}(A)=\rho_{4}(A) T_{2}=A T_{2}
$$

so $T_{2} \in \mathfrak{U}\left(\mathscr{C}_{6}^{\prime}\right)^{\prime}=\mathfrak{A}\left(\mathscr{C}_{6}\right)^{-}$. If $A \in \mathfrak{A}\left(\mathscr{C}_{6}\right)$ then

$$
T_{1} A=T_{1} \rho_{1}(A)=\rho_{3}(A) T_{1}=A T_{1}
$$

so $T_{1} \in \mathfrak{U}\left(\mathscr{C}_{6}\right)^{\prime}$. Hence $T_{1} T_{2}=T_{2} T_{1}$. We prove the result by reduction to this special case. Replace $T_{2}$ by $T_{3}:=T_{2} \circ U$, where $U \in\left(\rho_{0}, \rho_{2}\right)$ is unitary, $\rho_{0} \in \Delta\left(\mathscr{C}_{0}\right)$ and there exists a spacelike cone $\mathscr{C}_{6} \subset \mathscr{C}_{1}^{\prime} \cap \mathscr{C}_{3}^{\prime}, \mathscr{C}_{6} \supset \mathscr{C}_{0} \cup \mathscr{C}_{2}$, then

$$
T_{1} T_{3}=T_{1} T_{2} U ; \quad T_{3} T_{1}=T_{2} U T_{1}=T_{2} T_{1} U
$$

Thus $T_{1}$ and $T_{2}$ commute if and only if $T_{1}$ and $T_{3}$ commute. Now suppose $\mathscr{C}_{1}^{\prime} \cap \mathscr{C}_{3}^{\prime}$ is spacelike cone connected, then a finite number of such moves reduce us to the special case treated above. Hence $T_{1} T_{2}=T_{2} T_{1}$ whenever $\mathscr{C}_{1}^{\prime} \cap \mathscr{C}_{3}^{\prime}$ is spacelike cone connected. Now pick $U_{1} \in\left(\rho_{1}, \hat{\rho}_{1}\right)$ and $U_{3} \in\left(\rho_{3}, \hat{\rho}_{3}\right)$ unitary where $\hat{\rho}_{i} \in \Delta_{t}\left(\hat{\mathscr{C}}_{i}\right), i=1,3$, $\hat{\mathscr{C}}_{i} \subset \mathscr{C}_{i}$ and $\hat{\mathscr{C}}_{1} \cap \hat{\mathscr{C}}_{3}^{\prime}$ is spacelike cone connected. This is possible by Lemma A.7 and e) of the Appendix. Then $U_{3} \circ T_{1} \circ U_{1}^{*} \in\left(\hat{\rho}_{1}, \hat{\rho}_{3}\right)$ so $U_{3} T_{1} U_{1}^{*} T_{2}=T_{2} U_{3} T_{1} U_{1}^{*}$. But also $U_{1}^{*} T_{2}=T_{2} U_{1}^{*}$ and $U_{3} T_{2}=T_{2} U_{3}$ since $\mathscr{C}_{1}^{\prime}$ and $\mathscr{C}_{3}^{\prime}$ are spacelike cone connected. Hence $T_{1} T_{2}=T_{2} T_{1}$ as required.

\footnotetext{
27 In this section, we will identify $\mathfrak{A}$ with $\pi_{0}(\mathfrak{U})$ and omit the symbol $\pi_{0}$ except where special emphasis is desired
} 
Our next goal is to define $\pi(T)$ unambiguously when $\pi \in(S)$ and $T \in\left(\rho_{1}, \rho_{2}\right)$, $\rho_{i} \in \Delta_{t}\left(\mathscr{C}_{i}\right), i=1,2$. We pick a unitary $V \in(\rho, \pi)$, where $\rho \in \Delta_{t}(\mathscr{C})$ and $\mathscr{C} \subset \mathscr{C}_{1}^{\prime} \cap \mathscr{C}_{2}^{\prime}$ and set

$$
\pi(T):=V T V^{*}
$$

Again this should be the correct definition since $\rho$ should act trivially on $T$.

4.3 Lemma. $\pi(T)$ is independent of the choice of $V$ under the stated conditions.

Proof. If $V^{\prime} \in\left(\rho^{\prime}, \pi\right), \rho^{\prime} \in \Delta_{t}\left(\mathscr{C}_{0}\right)$ and $\mathscr{C}_{0} \subset \mathscr{C}_{1}^{\prime} \cap \mathscr{C}_{2}^{\prime}$ then $V^{*} V^{\prime} \in\left(\rho^{\prime}, \rho\right)$ and hence, by Lemma $4.2, V^{*} V^{\prime} T=T V^{*} V^{\prime}$, but this gives $V^{\prime} T V^{*}=V T V^{*}$ s required. We must still allow for the possibility that $\pi(T)$ might depend on the localization cones assigned to $\rho_{1}$ and $\rho_{2}$. It will clearly be enough to consider two different localization regions for $\rho_{1}$, say $\rho_{1} \in \Delta_{t}\left(\mathscr{C}_{1}\right) \cap \Delta_{t}\left(\hat{\mathscr{C}}_{1}\right)$. The problem is still not trivial because it is not clear whether $\mathscr{C}_{1}^{\prime} \cap \mathscr{C}_{2}^{\prime} \cap \mathscr{C}_{3}^{\prime}$ contains a spacelike cone. We proceed as follows: pick unitaries $V \in(\rho, \pi), \hat{V} \in(\hat{\rho}, \pi)$, where $\rho \in \Delta_{t}(\mathscr{C}), \mathscr{C} \subset \mathscr{C}_{1}^{\prime} \cap \mathscr{C}_{2}^{\prime}$ and $\hat{\rho} \in \Delta_{t}(\hat{\mathscr{C}})$, $\hat{\mathscr{C}} \subset \hat{\mathscr{C}}_{1}^{\prime} \cap \mathscr{C}_{2}^{\prime}$. We have to show that $V T V^{*}=\hat{V} T \hat{V}^{*}$. By f) of the Appendix, we can find spacelike cones $\mathscr{C}_{3}, \hat{\mathscr{C}}_{3}$ and $\mathscr{C}_{4}$ with $\mathscr{C}_{3} \subset \mathscr{C}_{1}, \hat{\mathscr{C}}_{3} \subset \hat{\mathscr{C}}_{1}$ and $\mathscr{C}_{4} \subset \mathscr{C}_{2}$ such that $\mathscr{C}_{3}^{\prime} \cap \hat{\mathscr{C}}_{3}^{\prime} \cap \mathscr{C}_{4}^{\prime}$ contains a spacelike cone. Pick unitaries $U_{1} \in\left(\rho_{3}, \rho_{1}\right)$, $U_{2} \in\left(\rho_{4}, \rho_{2}\right)$ and $\hat{U}_{1} \in\left(\hat{\rho}_{3}, \rho_{1}\right)$ with $\rho_{3} \in \Delta_{t}\left(\mathscr{C}_{3}\right), \rho_{4} \in \Delta_{t}\left(\mathscr{C}_{4}\right)$ and $\hat{\rho}_{3} \in \Delta_{t}\left(\hat{\mathscr{C}}_{3}\right)$. Pick $\mathscr{C}_{5} \subset \mathscr{C}_{3}^{\prime} \cap \hat{\mathscr{C}}_{3}^{\prime} \cap \mathscr{C}_{4}^{\prime}$ and a unitary $W \in(\tilde{\rho}, \pi)$ with $\tilde{\rho} \in \Delta_{t}\left(\mathscr{C}_{5}\right)$. Then we have $V U_{2}^{*} T U_{1} V^{*}=W U_{2}^{*} T U_{1} W^{*}$. Hence $V T V^{*}=V U_{2} V^{*} W U_{2}^{*} T U_{1} W^{*} V U_{1}^{*} V^{*}$ and similarly $\hat{V} T \hat{V}^{*}=\hat{V} U_{2} \hat{V}^{*} W U_{2}^{*} T \hat{U}_{1} W^{*} \hat{V} \hat{U}_{1}^{*} \hat{V}^{*}$. Now $V U_{2} V^{*}=\hat{V} U_{2} \hat{V}^{*}$ by Lemma 4.2. Pick a unitary $X \in(\tilde{\rho}, \pi)$, where $\tilde{\rho} \in \Delta_{t}(\tilde{\mathscr{C}})$ and $\tilde{\mathscr{C}} \subset \mathscr{C}_{1}^{\prime} \cap \hat{\mathscr{C}}_{1}^{\prime}$, then $V U_{1}^{*} V^{*}=$ $X U_{1}^{*} X^{*}$ and $\hat{V} \hat{U}_{1}^{*} \hat{V}^{*}=X \hat{U}_{1}^{*} X^{*}$ both by Lemma 4.1. Finally, $U_{1}^{*} \hat{U}_{1} W^{*} X=$ $W^{*} X U_{1}^{*} \hat{U}_{1}$ again by Lemma 4.1. A trivial computation now yields $V T V^{*}=\hat{V} T \hat{V}^{*}$ completing the proof.

It is obvious that $T \mapsto \pi(T)$ is linear and that $\pi(T)^{*}=\pi\left(T^{*}\right)$ but $\pi$ also preserves the composition of intertwiners.

4.4 Lemma. Let $T \in\left(\rho_{1}, \rho_{2}\right), S \in\left(\rho_{2}, \rho_{3}\right)$ and $\pi \in(S)$ then

$$
\pi(S \circ T)=\pi(S) \pi(T) .
$$

Proof. Let $\rho_{i} \in \Delta_{t}\left(\mathscr{C}_{i}\right), i=1,2,3$, then, by f) of the Appendix, we can pick unitaries $U_{i} \in\left(\rho_{i}, \hat{\rho}_{i}\right)$, where $\hat{\rho}_{i} \in \Delta_{t}\left(\hat{\mathscr{C}}_{i}\right), \quad \hat{\mathscr{C}}_{i} \subset \mathscr{C}_{i}$ and $\hat{\mathscr{C}}_{1}^{\prime} \cap \hat{\mathscr{C}}_{2}^{\prime} \cap \hat{\mathscr{C}}_{3}^{\prime}$ contains a spacelike cone. Let $S^{\prime}=U_{3} \circ S \circ U_{2}^{*}$ and $T^{\prime}=U_{2}^{\circ} \circ \circ U_{1}^{*}$, then using the spacelike cone in $\hat{\mathscr{C}}_{1}^{\prime} \cap \hat{\mathscr{C}}_{2}^{\prime} \cap \hat{\mathscr{C}}_{3}^{\prime}$, we can conclude that

$$
\pi\left(S^{\prime} \circ T^{\prime}\right)=V S^{\prime} T^{\prime} V^{*}, \quad \pi\left(S^{\prime}\right)=V S^{\prime} V^{*}, \quad \pi\left(T^{\prime}\right)=V T^{\prime} V^{*},
$$

thus $\pi\left(S^{\prime} \circ T^{\prime}\right)=\pi\left(S^{\prime}\right) \pi\left(T^{\prime}\right)$. Now picking a spacelike cone in $\hat{\mathscr{C}}_{3}^{\prime} \cap \mathscr{C}_{3}^{\prime} \cap \hat{\mathscr{C}}_{2}^{\prime} \cap \mathscr{C}_{2}^{\prime}=$ $\mathscr{C}_{3}^{\prime} \cap \mathscr{C}_{2}^{\prime}$ we can similarly conclude that $\pi\left(U_{3} \circ S \circ U_{2}^{*}\right)=\pi\left(U_{3}\right) \pi(S) \pi\left(U_{2}^{*}\right)$. Similarly, we get $\pi\left(U_{2} \circ T \circ U_{1}^{*}\right)=\pi\left(U_{2}\right) \pi(T) \pi\left(U_{1}^{*}\right)$ and $\pi\left(U_{3}^{*} \circ\left(S^{\prime} \circ T^{\prime}\right) \circ U_{1}\right)=\pi\left(U_{3}^{*}\right) \pi\left(S^{\prime} \circ T^{\prime}\right) \pi\left(U_{1}\right)$. Hence

as required.

$$
\begin{aligned}
\pi(S) \pi(T) & =\pi\left(U_{3}\right) * \pi\left(U_{3} \circ S \circ U_{2}^{*}\right) \pi\left(U_{2}\right) \pi\left(U_{2}\right)^{*} \pi\left(U_{2} \circ T \circ U_{1}^{*}\right) \pi\left(U_{1}\right) \\
& =\pi\left(U_{3}\right)^{*} \pi\left(S^{\prime}\right) \pi\left(T^{\prime}\right) \pi\left(U_{1}\right)=\pi\left(U_{3}\right)^{*} \pi\left(S^{\prime} \circ T^{\prime}\right) \pi\left(U_{1}\right) \\
& =\pi\left(U_{3}^{*} \circ S^{\prime} \circ T^{\prime} \circ U_{1}\right)=\pi(S \circ T),
\end{aligned}
$$


4.5 Lemma. Let $\pi_{1}, \pi_{2} \in(S)$, and $\rho \in \Delta_{t}(\mathscr{C})$ and $S \in\left(\pi_{1}, \pi_{2}\right)$ then $S \in\left(\pi_{1} \rho, \pi_{2} \rho\right)$.

Proof. It is enough to show that if $A \in \mathfrak{U}(\mathcal{O})$, then $S \pi_{1} \rho(A)=\pi_{2} \rho(A) S$. Pick a spacelike cone $\hat{\mathscr{C}} \subset \mathscr{C}^{\prime} \cap \mathcal{O}^{\prime}$ and unitaries $V_{i} \in\left(\rho_{i}, \pi_{i}\right), i=1,2$ with $\rho_{i} \in \Delta_{t}(\hat{\mathscr{C}})$. Let $B \in \mathfrak{U}(\hat{\mathscr{C}})$, then $\rho(A) B=\rho(A B)=\rho(B A)=B \rho(A)$ so that $\rho(A) \in \mathfrak{A}(\hat{\mathscr{C}})^{\prime}$. By definition, $\pi_{i} \rho(A)=V_{i} \rho(A) V_{i}^{*}, \quad i=1,2$ and $V_{2}^{*} \circ S \circ V_{1} \in\left(\rho_{1}, \rho_{2}\right)$ so $V_{2}^{*} \circ S \circ V_{1} \in \mathfrak{U}(\hat{\mathscr{C}})^{-}$, thus $\rho(A) V_{2}^{*} \circ S \circ V_{1}=V_{2}^{*} \circ S \circ V_{1} \rho(A)$ and rearranging we see that $S \pi_{1} \rho(A)=\pi_{2} \rho(A) S$ as required.

4.6 Lemma. Let $\pi \in(S)$ and $S \in\left(\rho_{1}, \rho_{2}\right), \rho_{i} \in \Delta_{t}\left(\mathscr{C}_{i}\right), i=1,2$, then $\pi(S) \in\left(\pi \rho_{1}, \pi \rho_{2}\right)$.

Proof. It is again enough to show that if $A \in \mathfrak{A}(\mathcal{O})$ then $\pi(S) \pi \rho_{1}(A)=\pi \rho_{2}(A) \pi(S)$. Pick a spacelike cone $\mathscr{C} \subset \mathscr{C}_{1}^{\prime} \cap \mathscr{C}_{2}^{\prime} \cap \mathcal{O}^{\prime}$; this is possible by Lemma A.5 and d) of the Appendix. Pick a unitary $V \in(\rho, \pi), \rho \in \Delta_{t}(\mathscr{C})$, then, by definition, $\pi(S)=V S V^{*}$. Since $\rho_{i}(A) \in \mathfrak{A}\left(\mathscr{C}^{\prime}\right)^{-}, i=1,2$, we also have $\pi \rho_{1}(A)=V \rho_{1}(A) V^{*}, \pi \rho_{2}(A)=V \rho_{2}(A) V^{*}$. Hence

$$
\pi(S) \pi \rho_{1}(A)=V S \rho_{1}(A) V^{*}=V \rho_{2}(A) S V^{*}=\pi \rho_{2}(A) \pi(S)
$$

as required.

4.7 Lemma. Given $\pi_{1}, \pi_{2} \in(S), S \in\left(\pi_{1}, \pi_{2}\right), \rho_{i} \in \Delta_{t}\left(\mathscr{C}_{i}\right), i=1,2$ and $T \in\left(\rho_{1}, \rho_{2}\right)$ then

$$
S \times T:=S \pi_{1}(T)=\pi_{2}(T) S
$$

defines an element of $\left(\pi_{1} \rho_{1}, \pi_{2} \rho_{2}\right)$.

Proof. Pick a spacelike cone $\mathscr{C} \subset \mathscr{C}_{1}^{\prime} \cap \mathscr{C}_{2}^{\prime}$ and unitaries $V_{i} \in\left(\hat{\rho}_{i}, \pi_{i}\right)$ with $\hat{\rho}_{i} \in \Delta_{t}(\mathscr{C})$, $i=1,2$. Then $T \in \mathfrak{A}(\mathscr{C})^{\prime}$ and $V_{2}^{*} \circ S \circ V_{1} \in\left(\hat{\rho}_{1}, \hat{\rho}_{2}\right)$ so $V_{2}^{*} S V_{1} \in \mathfrak{U}\left(\mathscr{C}^{\prime}\right)^{\prime}=\mathfrak{A}(\mathscr{C})^{-}$. Hence $T V_{2}^{*} S V_{1}=V_{2}^{*} S V_{1} T$ and thus $V_{2} T V_{2}^{*} S=S V_{1} T V_{1}^{*}$ or, by definition, $\pi_{2}(T) S=$ $S \pi_{1}(T)$. Now, by Lemma 4.5, $S \in\left(\pi_{1} \rho_{2}, \pi_{2} \rho_{2}\right)$ and, by Lemma 4.6, $\pi_{1}(T) \in\left(\pi_{1} \rho_{1}, \pi_{1} \rho_{2}\right)$ thus $S \pi_{1}(T) \in\left(\pi_{1} \rho_{1}, \pi_{2} \rho_{2}\right)$ as required.

4.8 Corollary. Let $\pi \in(S)$ and $\rho \in \Delta_{t}(\mathscr{C})$ then $\pi \rho \in(S)$. In particular if $\rho_{1}, \rho_{2} \in \Delta_{t}(\mathscr{C})$ then $\rho_{1} \rho_{2} \in \Delta_{t}(\mathscr{C})$.

Proof. Given any spacelike cone $\hat{\mathscr{C}}$ pick unitaries $V_{1} \in\left(\rho_{1}, \pi\right)$ and $V_{2} \in\left(\rho_{2}, \rho\right)$ with $\rho_{1}, \rho_{2} \in \Delta_{t}(\hat{\mathscr{C}})$ then $V_{1} \times V_{2} \in\left(\rho_{1} \rho_{2}, \pi \rho\right)$ is unitary and $\rho_{1} \rho_{2}$ is localized in $\widehat{\mathscr{C}}$. Hence $\pi \rho \in(S)$.

4.9 Lemma. Let $\pi_{1}, \pi_{2}, \pi_{3} \in(S), \quad S_{1} \in\left(\pi_{1}, \pi_{2}\right), \quad S_{2} \in\left(\pi_{2}, \pi_{3}\right), \quad \rho_{i} \in \Delta_{t}\left(\mathscr{C}_{i}\right), \quad i=1,2,3$, $T_{1} \in\left(\rho_{1}, \rho_{2}\right) ; T_{2} \in\left(\rho_{2}, \rho_{3}\right)$ then

$$
\left(S_{2} \circ S_{1}\right) \times\left(T_{2} \circ T_{1}\right)=S_{2} \times T_{2} \circ S_{1} \times T_{1} .
$$

Proof. $S_{2} S_{1} \pi_{1}\left(T_{2} \circ T_{1}\right)=S_{2} S_{1} \pi_{1}\left(T_{2}\right) \pi_{1}(T)$ by Lemma 4.4. $S_{1} \pi_{1}\left(T_{2}\right)=\pi_{2}\left(T_{2}\right) S_{1}$ by Lemma 4.7. Thus $S_{2} S_{1} \pi_{1}\left(T_{2} \circ T_{1}\right)=S_{2} \pi_{2}\left(T_{2}\right) S_{1} \pi_{1}\left(T_{1}\right)=S_{2} \times T_{2} \circ S_{1} \times T_{1}$ as required.

Let $\mathscr{S}$ denote the category of representations in $(S)$ and their intertwiners and $\mathscr{T}$ (respectively $\mathscr{T}(\mathscr{C})$ ) the full subcategory defined by those representations which are localized in some spacelike cone (respectively in the spacelike cone $\mathscr{C}$ ). Then the intertwiner calculus developed up to this point may be summed up in the following theorem. 
4.10 Theorem. There is $a^{*}$-functor $G$ of two variables

$$
G: \mathscr{S} \times \mathscr{T} \rightarrow \mathscr{S}
$$

defined by $G(\pi, \rho)=\pi \rho, G(S, T)=S \times T$.

Proof. The only points which have not been explicitly checked are the bilinearity of $G(S, T)$ and the self-adjointness of $G, G\left(S^{*}, T^{*}\right)=G(S, T)^{*}$. But these properties hold trivially.

Let us now restrict our attention to a fixed spacelike cone $\mathscr{C}$; we then claim that $\mathscr{T}(\mathscr{C})$ becomes a strict monoidal $C^{*}$-category. What we still have to verify is that $\times$ regarded as a functor $\mathscr{T}(\mathscr{C}) \times \mathscr{T}(\mathscr{C}) \rightarrow \mathscr{T}(\mathscr{C})$ is associative and that $l$, the identity representation $\pi_{0}$, is a monoidal unit. Given $T_{i} \in\left(\rho_{i}, \rho_{i}^{\prime}\right), \rho_{i}, \rho_{i}^{\prime} \in \Delta_{t}(\mathscr{C})$, $i=1,2,3$ and a double cone $\mathcal{O}$, pick a spacelike cone $\hat{\mathscr{C}} \subset \mathscr{C}^{\prime} \cap \mathcal{O}^{\prime}$ and then spacelike cones $\mathscr{C}_{1}, \mathscr{C}_{2}$ with $\mathscr{C}_{1} \cup \mathscr{C}_{2} \subset \hat{\mathscr{C}}$ and $\mathscr{C}_{1} \subset \mathscr{C}_{2}^{\prime}$. Pick unitaries $V_{i} \in\left(\hat{\rho}_{i}, \rho_{i}\right)$ with $\hat{\rho}_{i} \in \Delta_{t}\left(\mathscr{C}_{i}\right), i=1,2$. Then $V_{1} \times V_{2} \in\left(\hat{\rho}_{1} \hat{\rho}_{2}, \rho_{1} \rho_{2}\right)$ and $\hat{\rho}_{1} \hat{\rho}_{2} \in \Delta_{t}(\hat{\mathscr{C}}) . V_{1} \times V_{2}=\rho_{1}\left(V_{2}\right) V_{1}$ and since $\mathscr{C}_{1} \subset \mathscr{C}_{2}^{\prime} \cap \mathscr{C}^{\prime}, V_{1} \times V_{2}=V_{1} V_{2} V_{1}^{*} V_{1}=V_{1} V_{2}$. Now if $A \in \mathfrak{A}(\mathcal{O})$,

$$
\begin{aligned}
\left(\rho_{1} \rho_{2}\right)\left(\rho_{3}(A)\right) & =V_{1} V_{2} \rho_{3}(A) V_{2}^{*} V_{1}^{*}=V_{1} \rho_{2} \rho_{3}(A) V_{1}^{*}=\rho_{1}\left(\rho_{2} \rho_{3}(A)\right), \\
\left(T_{1} \times T_{2}\right) \times T_{3} & =T_{1} \rho_{1}\left(T_{2}\right) \rho_{1} \rho_{2}\left(T_{3}\right)=T_{1} V_{1} T_{2} V_{1}^{*} V_{1} V_{2} T_{3}\left(V_{1} V_{2}\right)^{*} \\
& =T_{1} V_{1} T_{2} V_{2} T_{3} V_{2}^{*} V_{1}^{*} \\
& =T_{1} V_{1}\left(T_{2} \rho_{3}\left(T_{2}\right)\right) V_{1}^{*}=T_{1} \rho_{1}\left(T_{2} \rho_{3}\left(T_{3}\right)\right) \\
& =T_{1} \times\left(T_{2} \times T_{3}\right) .
\end{aligned}
$$

Hence $\times$ is associatve. Obviously $\rho \imath=\imath \rho=\rho$ and $1, \times T=T \times 1_{\imath}=T$ so that $l$ acts as a monoidal unit.

Remark. The above proof of associativity does not require $\rho_{1}, \rho_{1}^{\prime} \in \Delta_{t}(\mathscr{C})$ so it shows that we have an action of $\mathscr{T}(\mathscr{C})$ on $\mathscr{S}, \mathscr{S} \times \mathscr{T}(\mathscr{C}) \rightarrow \mathscr{S}$.

4.11 Theorem. $\mathscr{T}(\mathscr{C})$ is a strict monoidal $C^{*}$-category and if $\mathscr{C}_{1} \subset \mathscr{C}_{2}$ then the inclusion of $\mathscr{T}\left(\mathscr{C}_{1}\right)$ in $\mathscr{T}\left(\mathscr{C}_{2}\right)$ is a strict monoidal $*$-functor and each object of $\mathscr{T}\left(\mathscr{C}_{2}\right)$ is unitarily equivalent to an object of $\mathscr{T}\left(\mathscr{C}_{1}\right)$, i.e. the inclusion is a relaxed unitary monoidal equivalence.

Proof. We have just verified that $\mathscr{T}(\mathscr{C})$ is a strict monoidal $C^{*}$-category. The inclusion of $\mathscr{T}\left(\mathscr{C}_{1}\right)$ in $\mathscr{T}\left(\mathscr{C}_{2}\right)$ is trivially a strict monoidal $*$-functor and each object of $\mathscr{T}\left(\mathscr{C}_{2}\right)$ is unitarily equivalent to an object of $\mathscr{T}\left(\mathscr{C}_{1}\right)$.

We turn now to questions of permutation symmetry. The basic lemma is

4.12 Lemma. Let $\rho_{i} \in \Delta_{t}\left(\mathscr{C}_{i}\right), i=1,2,3,4$ and $T_{1} \in\left(\rho_{1}, \rho_{3}\right)$ and $T_{2} \in\left(\rho_{2}, \rho_{4}\right)$ and suppose $\mathscr{C}_{1} \subset \mathscr{C}_{2}^{\prime}, \mathscr{C}_{3} \subset \mathscr{C}_{4}^{\prime}$, then $T_{1} \times T_{2}=T_{2} \times T_{1}$.

Proof. Suppose first that $\mathscr{C}_{2} \cup \mathscr{C}_{4} \subset \mathscr{C}_{1}^{\prime} \cap \mathscr{C}_{3}^{\prime}$ then $T_{1} \rho_{1}\left(T_{2}\right)=T_{1} T_{2}$ and $T_{2} \rho_{2}\left(T_{1}\right)=$ $T_{2} T_{1}$ by definition. But by Lemma 4.2, $T_{1} T_{2}=T_{2} T_{1}$ so we just need to prove that $\rho_{1} \rho_{2}=\rho_{2} \rho_{1}$ and $\rho_{3} \rho_{4}=\rho_{4} \rho_{3}$. Pick $\hat{\mathscr{C}}_{1} \subset \mathscr{C}_{2}^{\prime} \cap \mathcal{O}^{\prime}$ and $\hat{\mathscr{C}}_{2} \subset \hat{\mathscr{C}}_{1}^{\prime} \cap \mathscr{C}_{1}^{\prime} \cap \mathcal{O}^{\prime}$ and unitaries $V_{i} \in\left(\hat{\rho}_{i}, \rho_{i}\right)$ with $\hat{\rho}_{i} \in \Delta_{t}\left(\mathscr{C}_{i}\right), i=1,2$. Then if $A \in \mathfrak{U}(\mathcal{O})$,

$$
\begin{aligned}
& \rho_{1} \rho_{2}(A)=V_{1} \rho_{2}(A) V_{1}^{*}=V_{1} V_{2} A V_{2}^{*} V_{1}^{*} ; \\
& \rho_{2} \rho_{1}(A)=V_{2} \rho_{1}(A) V_{2}^{*}=V_{2} V_{1} A V_{1}^{*} V_{2}^{*} .
\end{aligned}
$$


But $\mathscr{C}_{2} \cup \hat{\mathscr{C}}_{2} \subset \mathscr{C}_{1}^{\prime} \cap \hat{\mathscr{C}}_{1}^{\prime}$ so Lemma 4.2 again shows that $V_{1} V_{2}=V_{2} V_{1}$. Hence $\rho_{1} \rho_{2}=\rho_{2} \rho_{1}$ and similarly $\rho_{3} \rho_{4}=\rho_{4} \rho_{3}$. In the general case, pick a unitary $U \in\left(\rho_{0}, \rho_{2}\right)$, where $\rho_{0} \in \Delta_{t}\left(\mathscr{C}_{0}\right)$ and $\mathscr{C}_{0} \cup \mathscr{C}_{2} \subset \mathscr{C}_{1}^{\prime}$, then if $T_{3}=T_{2} \circ U$,

$$
\begin{aligned}
& T_{1} \times T_{3}=T_{1} \times\left(T_{2} \circ U\right)=T_{1} \times T_{2} \circ 1_{\rho_{1}} \times U ; \\
& T_{3} \times T_{1}=\left(T_{2} \circ U\right) \times T_{1}=T_{2} \times T_{1} \circ U \times 1_{\rho_{1}} .
\end{aligned}
$$

But the first part of the proof shows that $1_{\rho_{1}} \times U=U \times 1_{\rho_{1}}$ hence $T_{1} \times T_{3}=T_{3} \times T_{1}$ if and only if $T_{1} \times T_{2}=T_{2} \times T_{1}$. Hence, by a finite set of such moves, we see that we can assume that $\mathscr{C}_{2} \subset \mathscr{C}_{1}^{\prime} \cap \mathscr{C}_{3}^{\prime}$. Shifting $\mathscr{C}_{4}$, the left support of $T_{2}$, in the same way inside $\mathscr{C}_{3}^{\prime}$ we can also assume $\mathscr{C}_{4} \subset \mathscr{C}_{1}^{\prime} \cap \mathscr{C}_{3}^{\prime}$. Thus from the first part of the proof we can conclude that $T_{1} \times T_{2}=T_{2} \times T_{1}$ completing the proof.

We now define the permutation symmetry $\varepsilon$ : if $\rho_{1}$ and $\rho_{2}$ are two objects of $\mathscr{T}$ then pick unitaries $U_{i} \in\left(\hat{\rho}_{i}, \rho_{i}\right)$, where $\hat{\rho}_{1}$ and $\hat{\rho}_{2}$ can be localized in mutually spacelike cones and set

$$
\varepsilon\left(\rho_{1}, \rho_{2}\right):=U_{2} \times U_{1} \circ U_{1}^{*} \times U_{2}^{*} .
$$

4.13 Lemma. $\varepsilon\left(\rho_{1}, \rho_{2}\right)$ is independent of the choice of $U_{1}$ and $U_{2}$. If $R_{i} \in\left(\rho_{i}, \sigma_{i}\right)$, $i=1,2$, then

$$
\varepsilon\left(\sigma_{1}, \sigma_{2}\right) \circ R_{1} \times R_{2}=R_{2} \times R_{1} \circ \varepsilon\left(\rho_{1}, \rho_{2}\right) .
$$

Proof. This is a straightforward application of Lemma 4.12. If $U_{i}^{\prime} \in\left(\rho_{i}^{\prime}, \rho_{i}\right)$, where $\rho_{1}^{\prime}$ and $\rho_{2}^{\prime}$ have spacelike separated supports then by Lemma 4.12

$$
\left(U_{1}^{*} \circ U_{1}^{\prime}\right) \times\left(U_{2}^{*} \circ U_{2}^{\prime}\right)=\left(U_{2}^{*} \circ U_{2}^{\prime}\right) \times\left(U_{1}^{*} \circ U_{1}^{\prime}\right),
$$

and rearranging we get

$$
\left(U_{2} \times U_{1}\right) \circ\left(U_{1}^{*} \times U_{2}^{*}\right)=\left(U_{2}^{\prime} \times U_{1}^{\prime}\right) \circ\left(U_{1}^{\prime *} \times U_{2}^{\prime *}\right)
$$

as required. If $V_{i} \in\left(\hat{\sigma}_{i}, \sigma_{i}\right)$ are unitaries and $\hat{\sigma}_{1}$ and $\hat{\sigma}_{2}$ have spacelike separated supports then

$$
\left(V_{1}^{* \circ} \circ R_{1} \circ U_{1}\right) \times\left(V_{2}^{*} \circ R \circ U_{2}\right)=\left(V_{2}^{*} \circ R_{2} \circ U_{2}\right) \times\left(V_{1}^{*} \circ R_{1} \circ U_{1}\right),
$$

and rearranging gives $(4.10)$ as required.

4.14 Lemma. If $\rho, \sigma, \tau \in \Delta_{t}(\mathscr{C})$ then

$$
\begin{gathered}
\varepsilon(\rho, \sigma) \circ \varepsilon(\sigma, \rho)=1_{\sigma \rho}, \\
\varepsilon(\rho, \imath)=\varepsilon(l, \rho)=1_{\rho}, \\
\varepsilon(\rho, \sigma \tau)=1_{\sigma} \times \varepsilon(\rho, \tau) \circ \varepsilon(\rho, \sigma) \times 1_{\tau},
\end{gathered}
$$

so that $(\mathscr{T}(\mathscr{C}), \varepsilon)$ becomes a strict symmetric monoidal $C^{*}$-category.

Proof. $\varepsilon(\rho, \sigma) \circ \varepsilon(\sigma, \rho)=V \times U \circ U^{*} \times V^{*} \circ U \times V \circ V^{*} \times U^{*}=1_{\sigma \rho}, \varepsilon(\rho, l)=1_{l} \times 1_{\rho} \circ 1_{\rho} \times$ $1_{l}=1_{\rho}$. To prove the final identity, choose $U \in(\hat{\rho}, \rho), V \in(\hat{\sigma}, \sigma)$ and $W \in(\hat{t}, \tau)$ in such a way that $\hat{\rho}, \hat{\sigma}, \hat{\tau}$ have mutually spacelike supports within the fixed spacelike cone $\mathscr{C}$ so that we can compute within $\mathscr{T}(\mathscr{C})$, 


$$
\begin{aligned}
1_{\sigma} \times \varepsilon(\rho, \tau) \circ \varepsilon(\rho, \sigma) \times 1_{\tau} & =1_{\sigma} \times\left(W \times U \circ U^{*} \times W^{*}\right) \circ\left(V \times U \circ U^{*} \times V^{*}\right) \times 1_{\tau} \\
& =1_{\sigma} \times W \times U \circ 1_{\sigma} \times U^{*} \times W^{*} \circ V \times U \times 1_{\tau} \circ U^{*} \times V^{*} \times 1_{\tau} \\
& =(V \times W) \times U \circ U^{*} \times(V \times W)^{*} .
\end{aligned}
$$

But provided we have chosen $\hat{\rho}, \hat{\sigma}, \hat{\tau}$ so that $\hat{\rho}$ and $\hat{\sigma} \hat{\tau}$ have spacelike separated supports, the right-hand side is just $\varepsilon(\rho, \sigma \tau)$ completing the proof.

We can now define a notion of statistics: let $\Delta_{t}:=\bigcup_{\mathscr{C}} \Delta_{t}(\mathscr{C})$.

4.15 Theorem. For each $\rho \in \Delta_{t}$ there are unique unitary representations $\varepsilon_{\rho}^{(n)}$ of $\mathbf{P}_{n}$ in $\left(\rho^{n}, \rho^{n}\right)=\rho^{n}(\mathfrak{U})^{\prime}, n \in \mathbf{N}$ such that

$$
\varepsilon_{\rho}^{(n)}(p) \times \varepsilon_{\rho}^{\left(n^{\prime}\right)}\left(p^{\prime}\right)=\varepsilon_{\rho}^{\left(n+n^{\prime}\right)}\left(p \times p^{\prime}\right), \quad p \in \mathbf{P}_{n}, \quad p^{\prime} \in \mathbf{P}_{n^{\prime}},
$$

where $p \times p^{\prime}$ is the obvious permutation obtained by juxtaposition of $p$ and $p^{\prime}$ and

$$
\varepsilon_{\rho}^{(n)}((r, s))=\varepsilon\left(\rho^{r}, \rho^{s}\right), \quad r+s=n,
$$

where $(r, s)$ denotes the permutation

$$
\left(\begin{array}{cccccccc}
1 & 2 & \cdots & r & r+1 & r+2 & \cdots & r+s \\
s+1 & s+2 & \cdots & s+r & 1 & 2 & \cdots & s
\end{array}\right) .
$$

If $T \in\left(\rho, \rho^{\prime}\right)$ is an arrow of $\mathscr{T}(\mathscr{C})$ then

$$
\varepsilon_{\rho^{\prime}}^{(n)}(p) \circ T^{\times n}=T^{\times n} \circ \varepsilon_{\rho}^{(n)}(p), \quad p \in \mathbf{P}_{n} .
$$

Furthermore, the equivalence classes of $\varepsilon_{\rho}^{(n)}, n \in \mathbf{N}$, depend only on the equivalence class of $\rho$.

Proof. Let $\rho \in \Delta_{t}(\mathscr{C})$ then the existence and uniqueness of $\varepsilon_{\rho}^{(n)}$ within $\mathscr{T}(\mathscr{C})$ follows from the coherence theorem for strict symmetric monoidal $C^{*}$-categories, cf. [2; Appendix]. Alternatively, one may follow the arguments of [1; Sect. 4] taking care of work within $\mathscr{T}(\mathscr{C})$. Now it follows from (4.14) by induction on $n$ that $\varepsilon_{\rho}^{(n)}$ is independent of the choice of localization region for $\rho$; recall, that $\times$ and $\varepsilon$ are defined on all of $\mathscr{T}$. Given $U \in\left(\rho, \rho^{\prime}\right)$ unitary, where $\rho, \rho^{\prime} \in \Delta_{t}(\mathscr{C})$ then the unitary equivalence of $\varepsilon_{\rho}^{(n)}$ and $\varepsilon_{\rho^{\prime}}^{(n)}$ follows from (4.17). Since Minkowski space is spacelike cone connected, the equivalence class of $\varepsilon_{\rho}^{(n)}$ depends only on the equivalence class of $\rho$.

To analyze the equivalence classes of $\varepsilon_{\rho}^{(n)}, n \in \mathbf{N}$, we need a left inverse for $\rho$. We will work with a fixed $\mathscr{T}(\mathscr{C})$ and the following notion will be adequate for our purposes: a left inverse $\varphi$ for $\rho \in \Delta_{t}(\mathscr{C})$ is a completely positive ${ }^{28}$ linear map $\varphi: \mathscr{B}\left(\mathscr{H}_{0}\right) \rightarrow \mathscr{B}\left(\mathscr{H}_{0}\right)$ such that

$$
\begin{gathered}
\varphi\left(\rho \rho^{\prime}(A) B\right)=\rho^{\prime}(A) \varphi(B), \quad A \in \mathcal{U}, \quad \rho^{\prime} \in \Delta_{t}(\mathscr{C}), \quad B \in \mathscr{B}\left(\mathscr{H}_{0}\right), \\
\varphi(\rho(T) B)=T \varphi(B), \quad T \in\left(\rho_{1}, \rho_{2}\right), \quad \rho_{1}, \rho_{2} \in \Delta_{t}(\mathscr{C}), \quad B \in \mathscr{B}\left(\mathscr{H}_{0}\right), \\
\varphi(I)=I .
\end{gathered}
$$

\footnotetext{
28 I.e. $\varphi$ and its extensions entry by entry to the $n \times n$-matrices over $\mathscr{B}\left(\mathscr{H}_{0}\right)$ are positive for all $n$
} 
Note that, since a positive linear mapping is automatically self-adjoint, the analogues of (4.18) and (4.19) hold with the order of the factors reversed. Since $\varphi$ is completely positive

$$
\varphi\left(B^{*} B\right) \geqq \varphi(B)^{*} \varphi(B), \quad B \in \mathscr{B}\left(\mathscr{H}_{0}\right) .
$$

To show that there are left inverses for $\rho$, we proceed as follows: pick a sequence $\mathscr{C}_{k}$ of spacelike cones in $\mathscr{C}^{\prime}$ which tend spacelike to infinity in the sense that they are eventually spacelike to any $\mathcal{O} \in \mathscr{K}$. Pick a unitary $U_{k} \in\left(\rho_{k}, \rho\right)$, where $\rho_{k} \in \Delta_{t}\left(\mathscr{C}_{k}\right)$, then the map $B \mapsto U_{k} B U_{k}^{*}$ is a unital completely positive map from $\mathscr{B}\left(\mathscr{H}_{0}\right)$ into $\mathscr{B}\left(\mathscr{H}_{0}\right)$. Now if $T \in\left(\rho_{1}, \rho_{2}\right), \rho_{1}, \rho_{2} \in \Delta_{t}(\mathscr{C})$ then $U_{k} \rho(T)=T U_{k}$, furthermore, if $A \in \mathfrak{U}$ then if $\rho^{\prime} \in \Delta_{t}(\mathscr{C})$

$$
\left\|U_{k} \rho \rho^{\prime}(A) B U_{k}^{*}-\hat{\rho}(A) U_{k} B U_{k}^{*}\right\| \rightarrow 0
$$

as $k \rightarrow \infty$. Indeed if $A \in \mathfrak{A}(\mathcal{O})$ then for $k$ sufficiently large we have $U_{k} \rho \rho^{\prime}(A)=\rho^{\prime}(A) U_{k}$, thus any limit point $\varphi$ of the mappings $B \mapsto U_{k} B U_{k}^{*}$ in the pointwise weak operator topology will be a completely positive linear map satisfying (4.18), (4.19) and (4.20). Such limit points exist since the set of unital completely positive linear maps from $\mathscr{B}\left(\mathscr{H}_{0}\right)$ to $\mathscr{B}\left(\mathscr{H}_{0}\right)$ is compact in the pointwise weak operator topology.

What interests us is the behaviour of $\varphi$ on the intertwiners between the powers of $\rho$. We have

4.16 Lemma. Let $\varphi$ be a left inverse for $\rho \in \Delta_{t}(\mathscr{C})$. If $S \in\left(\rho^{r+1}, \rho^{s+1}\right)$, then $\varphi(S) \in\left(\rho^{r}, \rho^{s}\right)$ and

$$
\varphi\left(S^{*} S\right) \geqq \varepsilon\left(\rho, \rho^{r}\right) \varphi(\varepsilon(\rho, \rho)) S^{*} S \varphi(\varepsilon(\rho, \rho)) \varepsilon\left(\rho, \rho^{r}\right)
$$

and, in particular,

$$
\left\|\varphi\left(S^{*} S\right)\right\| \geqq\|S \varphi(\varepsilon(\rho, \rho))\|^{2} .
$$

Proof. If $S \in\left(\rho^{r+1}, \rho^{s+1}\right)$ then, by (4.18)

$$
\varphi(S) \rho^{r}(A)=\varphi\left(S \rho^{r+1}(A)\right)=\varphi\left(\rho^{s+1}(A) S\right)=\rho^{s}(A) \varphi(S)
$$

so $\varphi(S) \in\left(\rho^{r}, \rho^{s}\right)$. Since, by $(4.10)$,

$$
S^{*} S=\varepsilon\left(\rho, \rho^{r+1}\right) \rho\left(S^{*} S\right) \varepsilon\left(\rho^{r+1}, \rho\right)=\rho\left(\varepsilon\left(\rho, \rho^{r}\right)\right) \varepsilon(\rho, \rho) \rho\left(S^{*} S\right) \varepsilon(\rho, \rho) \rho\left(\varepsilon\left(\rho^{r}, \rho\right)\right)
$$

by (4.13). Taking $B=\rho(S) \varepsilon(\rho, \rho) \rho\left(\varepsilon\left(\rho^{r}, \rho\right)\right)$ in (4.21) and using (4.19) we get (4.22) and hence (4.23).

With this lemma, we can employ $\varphi$ in a standard way to obtain a classification of statistics.

4.17 Theorem. If $\rho \in \Delta_{t}(\mathscr{C})$ is irreducible and $\varphi$ is a left inverse for $\rho$ then

$$
\varphi(\varepsilon(\rho, \rho))=\lambda I, \text { where } \lambda \in\left\{0, \pm d^{-1}: d \in \mathbf{N}\right\} .
$$

The Young tableaux associated with $\varepsilon_{\rho}^{(n)}$ are all Young tableaux

a) whose columns have length $\leqq d$ if $\lambda=d^{-1}$ (para-Bose statistics of order $d$ );

b) whose rows have length $\leqq d$ if $\lambda=-d^{-1}$ (para-Fermi statistics of order $d$ );

c) without restriction, if $\lambda=0$ (infinite statistics).

This is proved as [1; Theorem 5.5] with the minor modification that, when 
$\lambda \neq 0$, the faithfulness of $\varphi$ on intertwiners between powers of $\rho$ is deduced from (4.23). For reducible $\rho$, we introduce the concept of a standard left inverse being a left inverse such that $\varphi(\varepsilon(\rho, \rho))^{2}$ is a multiple of the unit. The existence of such a left inverse is proved as in [1; Proposition 6.3]. $\rho$ is said to have infinite statistics if there is a left inverse $\varphi$ with $\varphi(\varepsilon(\rho, \rho))=0$, otherwise $\rho$ has finite statistics.

The classification of statistics for reducible $\rho$ with finite statistics is given in

4.18 Theorem. If $\rho \in \Delta_{t}(\mathscr{C})$ has finite statistics and $\varphi$ is a standard left inverse for $\rho$ then

$$
d(\rho) \varphi(\varepsilon(\rho, \rho))=\kappa(\rho),
$$

where $d(\rho)$ is an integer, the statistical dimension of $\rho$ and $\kappa(\rho) \in(\rho, \rho)$ is of square $1_{\rho}$. Furthermore,

$$
d(\rho) \varphi(\kappa(\rho))=(b(\rho)-f(\rho)) 1_{\imath},
$$

where $b(\rho)$ and $f(\rho)$ are integers with $b(\rho)+f(\rho)=d(\rho)$. The Young tableaux associated with $\varepsilon_{\rho}^{(n)}$ are all Young tableaux with at most $b(\rho)$ rows of length $>f(\rho)$ and at most $f(\rho)$ columns of length $>b(\rho)$. If $\rho^{\prime} \in \Delta_{t}(\mathscr{C})$ also has finite statistics then so does $\rho \rho^{\prime}$ and

$$
\begin{gathered}
\kappa(\rho) \times \kappa\left(\rho^{\prime}\right)=\kappa\left(\rho \rho^{\prime}\right), \\
d\left(\rho \rho^{\prime}\right)=d(\rho) d\left(\rho^{\prime}\right) .
\end{gathered}
$$

If $S \in\left(\rho, \rho^{\prime}\right)$ then

$$
S \circ \kappa(\rho)=\kappa\left(\rho^{\prime}\right) \circ S .
$$

$\rho$ has finite statistics if and only if it is a finite direct sum of irreducibles with finite statistics.

A proof can be given along the lines of [1; Sect. 6] again using (4.23) to show that a standard left inverse of a $\rho \in \Delta_{t}(\mathscr{C})$ with finite statistics is faithful on intertwiners between powers of $\rho$.

The following result is crucial for the existence of conjugates for $\rho \in \Delta_{t}(\mathscr{C})$ with finite statistics.

4.19 Theorem. For an element $\gamma \in \Delta_{t}(\mathscr{C})$, the following are equivalent

a) $d(\gamma)=1$,

b) there exists $a \gamma^{-1} \in \Delta_{t}(\mathscr{C})$ with $\gamma \gamma^{-1}=\imath=\gamma^{-1} \gamma$.

Proof. If we can find $\gamma^{-1}$ then $d(\gamma) d\left(\gamma^{-1}\right)=d(\imath)=1$ so that $d(\gamma)=1$. Conversely, suppose $d(\gamma)=1$ then, if $\varphi$ is a left inverse for $\gamma, \varphi(\varepsilon(\gamma, \gamma) \mp I)=0$ according as $\gamma$ satisfies Bose or Fermi statistics, respectively. Thus $\varepsilon(\gamma, \gamma)=\mp I$ since $\varphi$ is faithful on intertwiners between powers of $\gamma$. Given $A \in \mathfrak{A}(\mathcal{O})$, pick a unitary $U \in\left(\gamma_{1}, \gamma\right)$ where $\gamma_{1} \in \Delta_{t}\left(\mathscr{C}_{1}\right)$ and $\mathscr{C}_{1} \subset \mathscr{C}^{\prime} \cap \mathcal{O}^{\prime}$, then

$$
A=\gamma_{1}(A)=U^{*} \gamma(A) U .
$$

Now, by (4.10), $\varepsilon(\gamma, \gamma) \gamma(U)=U$, i.e. $U=\mp \gamma(U)$. But then $A=\gamma\left(U^{*}\right) \gamma(A) \gamma(U)$ and we define

$$
\gamma^{-1}(A):=\varphi(A), \quad A \in \mathfrak{A}
$$


and see that for $A \in \mathfrak{A}(\mathcal{O})$,

$$
\gamma^{-1}(A)=U^{*} A U .
$$

If follows that $\gamma^{-1}(A B)=\gamma^{-1}(A) \gamma^{-1}(B), A, B \in \mathfrak{A}$ so that $\gamma^{-1}$ is a representation which is localized in $\mathscr{C}$. We want to show that $\gamma^{-1} \in \Delta_{t}(\mathscr{C})$ : pick $V \in\left(\gamma, \gamma^{\prime}\right)$ unitary with say $\gamma^{\prime} \in \Delta_{t}\left(\mathscr{C}_{0}\right)$ then picking $\mathscr{C}_{1} \subset \mathscr{C}^{\prime} \cap \mathscr{C}_{0}^{\prime} \cap \mathcal{O}^{\prime}$, we deduce that for $A \in \mathfrak{A}(\mathcal{O})$,

$$
\gamma^{\prime-1}(A)=U^{*} V^{*} A V U=U^{*} V^{*} U \gamma^{-1}(A) U^{*} V U .
$$

Now letting $\mathscr{C}_{1}$ tend spacelike to infinity and applying Lemma 4.2 we deduce that there is a unitary $W \in\left(\gamma^{-1}, \gamma^{-1}\right)$ and that $W=U^{*} V^{*} U$ whenever $U \in\left(\gamma_{1}, \gamma\right)$ is unitary and $\gamma_{1} \in \Delta_{t}\left(\mathscr{C}_{1}\right), \quad \mathscr{C}_{1} \subset \mathscr{C}^{\prime} \cap \mathscr{C}_{0}^{\prime}$. Hence $\gamma^{-1} \in \Delta_{t}(\mathscr{C})$. Now if $A \in \mathfrak{A}(\mathcal{O})$ and $\mathscr{C}_{1} \subset \mathscr{C}^{\prime} \cap \mathcal{O}^{\prime}$

$$
\gamma \gamma^{-1}(A)=U \gamma^{-1}(A) U^{*}=U U^{*} A U U^{*}=A .
$$

Thus $\gamma \gamma^{-1}=\imath$ and consequently $d\left(\gamma^{-1}\right)=1$. Hence there is a $\tilde{\gamma} \in \Delta_{t}(\mathscr{C})$ with $\gamma^{-1} \tilde{\gamma}=l$, but then multiplying on the left by $\gamma$ we get $\gamma=\tilde{\gamma}$ completing the proof.

Our final goal is to show that if $\rho \in \Delta_{t}(\mathscr{C})$ has finite statistics then it has a conjugate within $\Delta_{t}(\mathscr{C})$. We let $\Delta_{f}(\mathscr{C})$ denote the subsemigroup of $\Delta_{t}(\mathscr{C})$ consisting of elements with finite statistics. To express the notion of conjugate in as simple a manner as possible we introduce a "Bosonized" symmetry for the strict monoidal $C^{*}$-category $\mathscr{T}_{f}(\mathscr{C})$ whose objects are $\Delta_{f}(\mathscr{C})$. We set:

$$
\hat{\varepsilon}\left(\rho_{1}, \rho_{2}\right):=\varepsilon\left(\rho_{1}, \rho_{2}\right) \circ \delta n\left(\rho_{1}, \rho_{2}\right)=\delta n\left(\rho_{1}, \rho_{2}\right) \circ \varepsilon\left(\rho_{1}, \rho_{2}\right),
$$

where

$$
2 \delta n\left(\rho_{1}, \rho_{2}\right):=\left(1_{\rho_{1}} \times 1_{\rho_{2}}\right)+\left(1_{\rho_{1}} \times \kappa\left(\rho_{2}\right)\right)+\left(\kappa\left(\rho_{1}\right) \times 1_{\rho_{2}}\right)-\left(\kappa\left(\rho_{1}\right) \times \kappa\left(\rho_{2}\right)\right),
$$

and one checks easily that $\hat{\varepsilon}$ is a symmetry for $\mathscr{T}_{f}(\mathscr{C})$, i.e. that (3.15) to (3.18) hold.

We can now show

4.20 Theorem. $\left(\mathscr{T}_{f}(\mathscr{C}), \hat{\varepsilon}\right)$ has conjugates, i.e. given $\rho \in \Delta_{f}(\mathscr{C})$, there is a $\bar{\rho} \in \Delta_{f}(\mathscr{C})$ and an $R \in(l, \bar{\rho} \rho)$ such that

$$
\begin{aligned}
& \bar{R}^{*} \times 1_{\rho}^{\circ} 1_{\rho} \times R=1_{\rho} \\
& R^{*} \times 1_{\bar{\rho}} \circ 1_{\bar{\rho}} \times \bar{R}=1_{\bar{\rho}}
\end{aligned} \text { where } \quad \bar{R}=\hat{\varepsilon}(\bar{\rho}, \rho) \circ R .
$$

The proof follows that of [9; Theorem 3.3]. In fact, all the computations involved are just algebraic manipulations within the category $\mathscr{T}_{f}(\mathscr{C})$.

This completes our discussion of the intertwiner calculus for topological charges. The main theorem of [2] could again be invoked to show that $\mathscr{T}_{f}(\mathscr{C})$ is isomorphic to a category of the type $\mathbf{U}(G)$ for a compact group $G$ unique up to isomorphism.

Note that if $\mathscr{C}_{1} \subset \mathscr{C}_{2}$ the inclusion of $\left(\mathscr{T}\left(\mathscr{C}_{1}\right), \hat{\varepsilon}\right)$ in $\left(\mathscr{T}\left(\mathscr{C}_{2}\right), \hat{\varepsilon}\right)$ is a strict symmetric monoidal *-functor and a relaxed unitary monoidal equivalence (cf. Theorem 4.11) so that (cf. [2; Theorem 6.10]) $G$ is independent of $\mathscr{C}$. However, just as in the case of localizable charges, we shall construct $G$ directly, together with the field algebras associated with spacelike cones. This is the task for the next section. 


\section{The Gauge Group and Normal Field Operators for Topological Charges}

In this section we derive our main result on the existence of a compact group of internal symmetries describing the whole superselection structure, including both localizable and topological charges. Here too this group will act on field operators which obey normal commutation relations at spacelike separations, and this makes it unique. However the field operators can no longer be localized in bounded spacetime regions if there are indeed topological charges in the theory (cf. Theorem 3.6c); instead, like the representations $\rho$ of Sect. 4, they will be rather associated with spacelike cones. We shall therefore deal with von Neumann algebras $\mathfrak{F}(\mathscr{C})$ of field operators associated with spacelike cones $\mathscr{C}$.

In the case of localizable charges studied in Sect. $3, \mathscr{F}(\mathscr{C})$ can be defined as the von Neumann algebra generated by all $\mathfrak{F}(\mathcal{O}), \mathcal{O} \in \mathscr{K}, \mathcal{O} \subset \mathscr{C}$. In this case it is easily seen that its gauge invariant part is $\pi(\mathfrak{H}(\mathscr{C}))^{-}$, the weak closure of $\pi(\mathfrak{H}(\mathscr{C}))$.

This motivates the following variant of Definition 3.1.

5.1 Definition. Let $\pi_{0}$ denote the vacuum representation of the net $\mathfrak{A}$ of local observables, acting on the Hilbert space $\mathscr{H}_{0}$.

An extended ${ }^{29}$ field system with gauge symmetry $\{\pi, G, \mathfrak{F}\}$ consists of a representation $\pi$ of $\mathfrak{A}$ on a Hilbert space $\mathscr{H}$ containing $\pi_{0}$ as a subrepresentation on $\mathscr{H}_{0} \subset \mathscr{H}$, a (strongly) compact group $G$ of unitaries on $\mathscr{H}$ leaving $\mathscr{H}_{0}$ pointwise fixed, and an inclusion preserving map $\mathscr{C} \rightarrow \mathfrak{F}(\mathscr{C})$ assigning to each spacelike cone a von Neumann algebra acting on $\mathscr{H}$ such that

a) the $g \in G$ induce automorphisms $\alpha_{g}$ of $\left.\mathscr{F}(\mathscr{C})\right)$ with $\pi(\mathfrak{H}(\mathscr{C}))^{-}$as fixed-point subalgebras, $\mathscr{C} \in \mathscr{F}$;

B) for each ac $^{30} \in \mathscr{J}$, the union of $\mathfrak{F}(\mathscr{C}+a)$ over all spacetime translates of $\mathscr{C}$ is irreducible;

r) $\mathscr{H}_{0}$ is cyclic for each $\mathfrak{F}(\mathscr{C}), \mathscr{C} \in \mathscr{J}$;

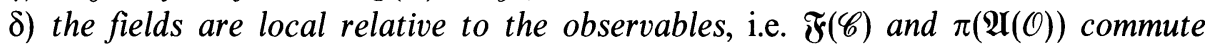
elementwise whenever the spacelike cone $\mathscr{C}$ and the double cone $\mathcal{O}$ are spacelike separated.

Since the field systems studied in Sect. 3 can only describe localizable charges, the definition of completeness given there was appropriate. Here, of course, we need a different definition:

5.2 Definition. An extended field system with gauge symmetry $\{\pi, G, \mathfrak{F}\}$ is complete if each equivalence class of irreducible representations of $\mathfrak{A}$ satisfying (1.2) and having finite statistics is realized as a subrepresentation of $\pi$, i.e. $\pi$ describes all charges, both localized and topological.

The definition of normal extended field systems with gauge symmetry reads as Definition 3.2 but for the obvious change that (3.2), (3.3) should now apply to $F \in \mathfrak{F}\left(\mathscr{C}_{1}\right), F^{\prime} \in \mathfrak{F}\left(\mathscr{C}_{2}\right)$ whenever $\mathscr{C}_{1}, \mathscr{C}_{2}$ are spacelike separated spacelike cones.

\footnotetext{
29 The field systems discussed in Sect. 3 might well have been termed local field systems to stress the fact that the field operators, unlike those studied here, can be localized in bounded and even arbitrarily small regions of spacetime.

${ }^{30}$ For what follows one might equivalently assume this property for just one $\mathscr{C} \in \mathscr{J}$
} 
The definition of equivalent extended field systems with gauge symmetry reads as Definition 3.4 but for the obvious modification of Eq. (3.6):

$$
W \mathfrak{F}_{1}(\mathscr{C})=\mathfrak{F}_{2}(\mathscr{C}) W, \quad \mathscr{C} \in \mathscr{J} .
$$

Our main results can now be stated and parallel Theorems 3.5, 3.6.

5.3 Theorem. Let $\mathfrak{A}$ be a net of local observables fulfilling property $B^{\prime}$ and suppose that duality (4.1) holds in the faithful irreducible vacuum representation $\pi_{0}$ acting on a separable ${ }^{31}$ Hilbert space $\mathscr{H}_{0}$. Then there exists a complete normal extended field system with gauge symmetry and this system is unique up to equivalence.

Given an extended field system and a double cone $D$ at spacelike infinity, we will denote by $\mathfrak{F}_{D}$ the $C^{*}$-algebra generated by all $\mathfrak{F}(\mathscr{C}), \mathscr{C} \in \mathscr{J}$ with $D(\mathscr{C}) \subset D$ (cf. Appendix). Thus $\beta$ ) of Definition 5.2 is equivalent to $\mathfrak{F}_{D}^{\prime \prime}=\mathscr{B}(\mathscr{H})$ for each $D$.

5.4 Theorem. Let $\{\pi, G, \mathfrak{F}\}$ be an extended field system with gauge symmetry; then, if $D$ is any double cone at spacelike infinity

a) $\pi(\mathscr{U})^{\prime} \cap \mathfrak{I}_{D}=\mathbf{C} \cdot I$

b) an automorphism $\gamma$ of the $C^{*}$-algebra $\mathfrak{F}_{D}$ is a gauge automorphism, i.e. $\gamma=\alpha_{g}$ for some $g \in G$, if and only if $\gamma$ acts trivially on $\pi(\mathfrak{U})$;

c) $\pi(\mathfrak{U})^{\prime}=G^{\prime \prime}$,

$$
\pi \cong \bigoplus_{\xi} d(\xi) \pi_{\xi}
$$

where the $\pi_{\xi}$ are inequivalent irreducible representations of $\mathfrak{A}$ fulfilling (1.2) with parastatistics of finite order $d(\xi)$.

If furthermore the sytem is normal, then the analogues of d), e) of Theorem 3.6 hold (with the obvious modification that twisted duality (Eq. (3.8), (3.9)) now refers to each $\mathscr{C} \in \mathscr{J}$ rather than $\mathcal{O} \in \mathscr{K})$.

The present results are the analogues of those of Sect. 3 with the spacelike cones $\mathscr{C}$ playing the role of double cones $\mathcal{O}$ as localization regions, and with $\pi(\mathfrak{U}(\mathscr{C}))^{\prime \prime}$ playing the role of the local algebras $\mathfrak{A}(\mathcal{O})$. However, $\mathscr{C}$ is now infinitely extended and, if we take the weak closure of $\pi(\mathfrak{A}(\mathscr{C}))$, we can expect that the $C^{*}$-algebra generated by all the $\pi(\mathfrak{U}(\mathscr{C}))^{\prime \prime}, \mathscr{C} \in \mathscr{J}$, contains global quantities like a total charge, since the $t=0$ part of spacelike infinity can be covered by finitely many double cones. This is the reason why $\mathfrak{F}_{D}$ appears in Theorem 5.4a), and we will further comment on this point at the end of this section.

In order to prove Theorem 5.3, the essential step going beyond the discussion of Sect. 3 is to establish the existence and uniqueness of a system $\left\{\pi, G, \rho \in \Delta_{f} \rightarrow H_{\rho}\right\}$ fulfilling properties similar to A)-H) of Sect. 3. Here we cannot apply [14; Corollary 6.2] directly since the elements of $\Delta_{f}$ do not leave the $C^{*}$-algebra $\mathfrak{A}$ stable nor can $\mathfrak{U}$ be extended in any useful way in order to achieve this property.

However we shall be able to apply those results if we first restrict our attention to a given double cone $D$ at spacelike infinity, so that each $\rho \in \Delta_{f}(\mathscr{C}), D(\mathscr{C}) \subset D$, defines an endomorphism of a fixed $C^{*}$-algebra $\mathfrak{U}_{D}$.

31 C.f. the remark following Lemma 3.11 
Let $\mathscr{J}(D)$ denote the set of all spacelike cones $\mathscr{C}$ such that $D(\mathscr{C}) \subset D, \Delta_{f}(D)$ the union of all $\Delta_{f}(\mathscr{C}), \mathscr{C} \in \mathscr{J}(D)$, and $\mathscr{T}_{f}(D)$ the full subcategory of $\mathscr{T}_{f}$ with objects $\Delta_{f}(D)$. Denote by $\mathfrak{A}_{D}$ the $C^{*}$-algebra generated by all $\mathfrak{A}(\mathscr{C})^{\prime \prime}, \mathscr{C} \in \mathscr{J}(D)$.

We will first embed $\mathscr{T}_{f}(D)$ in End $\mathfrak{A}_{D}$ as a full strict symmetric monoidal subcategory. This will allow us to apply [14; Corollary 6.2] to get a representation $\pi^{D}$ of $\mathfrak{A}_{D}$ on a Hilbert space $\mathscr{H} \supset \mathscr{H}_{0}$, a compact group $G$ of unitaries on $\mathscr{H}$, and a homomorphism $\rho \rightarrow H_{\rho}$ of $\Delta_{f}(D)$ into the semigroup of Hilbert spaces with support $I$ in $\mathscr{B}(\mathscr{H})$ fulfilling properties similar to $\mathrm{A})-\mathrm{H}$ ) of Sect. 3.

Next we will consider the restriction $\pi$ of $\pi^{D}$ to $\mathscr{A}$ and extend the map $\rho \rightarrow H_{\rho}$ to all of $\Delta_{f}$ in a unique way by means of the unitary intertwiners in $\mathscr{T}_{f}: H_{\rho}=\pi(U) H_{\rho_{1}}$ if $U \in\left(\rho_{1}, \rho\right), \rho_{1} \in \Delta_{f}(D)$.

In this way we shall get a system $\left\{\pi, G, \rho \in \Delta_{f} \rightarrow H_{\rho}\right\}$ fulfilling a slight variant of properties $\mathrm{A}$ ) $-\mathrm{H}$ ) and making no reference to a choice of a double cone $D$ at spacelike infinity. Changing $D$ in this construction would not change the system to within equivalence.

From this point on, the argument will proceed exactly as in Sect. 3: we will define $\mathfrak{F}(\mathscr{C})$ as the von Neumann algebra generated by the $H_{\rho}$ as $\rho$ runs through $\Delta_{f}(\mathscr{C})$.

The embedding of $\mathscr{T}_{f}(D)$ into End $\mathfrak{A}_{D}$ is made possible by the following lemma whose basic idea is close in spirit to the construction of [6; Lemma 4.1].

5.5 Lemma. Each $\rho \in \Delta_{t}(D)$ admits a unique extension to an endomorphism $\rho^{D}$ of $\mathfrak{A}_{D}$ which is normal in restriction to each $\mathfrak{A}(\mathscr{C})^{-}, \mathscr{C} \in \mathscr{J}(D)$. This extension is compatible with Eq. (4.5) in the following sense: if $\rho_{1}, \rho_{2} \in \Delta_{t}(D)$ and $T \in\left(\rho_{1}, \rho_{2}\right)$, then $T \in \mathfrak{A}_{D}$ and

$$
\rho^{D}(T)=\rho(T) \text {. }
$$

Furthermore

$$
\begin{aligned}
& \left(\rho_{1} \rho_{2}\right)^{D}=\rho_{1}^{D} \rho_{2}^{D}, \\
& \left(\rho_{1}, \rho_{2}\right)=\left(\rho_{1}^{D}, \rho_{2}^{D}\right)
\end{aligned}
$$

Proof. If $\rho \in \Delta_{t}(\mathscr{C}), D(\mathscr{C}) \subset D$ then given $\mathscr{C}_{1}$ with $D\left(\mathscr{C}_{1}\right) \subset D$ pick $\mathscr{C}_{2}$ with $D\left(\mathscr{C}_{2}\right) \subset D$ and $\mathscr{C}_{2} \supset \mathscr{C} \cup \mathscr{C}_{1}$. Let $A \in \mathfrak{A}\left(\mathscr{C}_{1}\right), B \in \mathfrak{A}\left(\mathscr{C}_{2}^{\prime}\right)$ then $B \rho(A)=\rho(B A)=\rho(A B)=\rho(A) B$ so $\rho(A) \in \mathfrak{A}\left(\mathscr{C}_{2}^{\prime}\right)^{\prime}=\mathfrak{H}\left(\mathscr{C}_{2}\right)^{-}$. Now pick a unitary $V \in\left(\rho^{\prime}, \rho\right)$, where $\rho^{\prime} \in \Delta_{t}\left(\mathscr{C}_{3}\right)$ and $\mathscr{C}_{3} \subset \mathscr{C}_{2}^{\prime}$ then if $A \in \mathfrak{A}\left(\mathscr{C}_{2}\right), \rho(A)=V A V^{-1}$ so $\rho$ admits a normal extension to a mapping of $\mathfrak{U}\left(\mathscr{C}_{2}\right)^{-}$into $\mathfrak{A}\left(\mathscr{C}_{2}\right)^{-}$. Thus $\rho$ admits a unique extension to an endomorphism $\rho^{D}$ of $\mathfrak{A}_{D}$ which is normal in restriction to each $\mathfrak{A}(\mathscr{C})^{-}, D(\mathscr{C}) \subset D$. If $\rho_{1}$ and $\rho_{2}$ are objects of $\mathscr{T}_{t}(D)$ then we may suppose $\rho, \rho_{1}, \rho_{2} \in \Delta_{t}(\mathscr{C}), D(\mathscr{C}) \subset D$ and if $T \in\left(\rho_{1}, \rho_{2}\right)$ then $T \in \mathfrak{A}\left(\mathscr{C}^{\prime}\right)^{\prime}=\mathfrak{U}(\mathscr{C})^{-}$. If we pick a unitary $V \in\left(\rho^{\prime}, \rho\right)$ with $\rho^{\prime} \in \Delta_{t}(\hat{\mathscr{C}}), \hat{\mathscr{C}} \subset \mathscr{C}^{\prime}$ we have

$$
\rho^{D}(T)=V T V^{*}=\rho(T) .
$$

Picking $\mathscr{C}_{1} \supset \mathscr{C}, D\left(\mathscr{C}_{1}\right) \subset D$, we have for $A \in \mathfrak{A}\left(\mathscr{C}_{1}\right)$,

$$
T \rho_{1}^{D}(A)=T \rho_{1}(A)=\rho_{2}(A) T=\rho_{2}^{D}(A) T .
$$

Since $\rho_{1}^{D}$ and $\rho_{2}^{D}$ are weakly continuous on each $\mathfrak{2}\left(\mathscr{C}_{1}\right)^{-}$, we conclude that $T \in\left(\rho_{1}^{D}, \rho_{2}^{D}\right)$. Now pick a unitary $U_{i} \in\left(\hat{\rho}_{i}, \rho_{i}\right)$ with $\hat{\rho}_{i} \in \Delta_{t}(\hat{\mathscr{C}}), i=1,2$ and $\hat{\mathscr{C}} \subset \mathscr{C}_{1}^{\prime}$, then 
if $A \in \mathfrak{U}\left(\mathscr{C}_{1}\right)$ we have

so

$$
\rho_{2}^{D}(A)=U_{2} A U_{2}^{*} \in \mathfrak{A}\left(\mathscr{C}_{1}\right)^{-}
$$

$$
\rho_{1}^{D} \rho_{2}^{D}(A)=U_{1} U_{2} A U_{2}^{*} U_{1}^{*}=U_{1} \rho_{2}(A) U_{1}^{*}=\rho_{1} \rho_{2}(A) .
$$

Thus $\rho_{1}^{D} \rho_{2}^{D}=\left(\rho_{1} \rho_{2}\right)^{D}$ since each is an extension of $\rho_{1} \rho_{2}$ normal on each $\mathfrak{A}\left(\mathscr{C}_{1}\right)$, $\mathscr{C}_{1} \in \mathscr{J}(D)$.

We may sum up the last result as

5.6 Corollary. The maps $\rho \rightarrow \rho^{D}, T \in\left(\rho_{1}, \rho_{2}\right) \rightarrow T \in \mathfrak{A}_{D}$ define a faithful strict monoidal *-functor of $\mathscr{T}_{t}(D)$ onto a full subcategory of End $\mathfrak{U}_{D}$.

Now $\left(\mathscr{T}_{f}(D), \hat{\varepsilon}\right)$ is a strict symmetric monoidal $C^{*}$-category with conjugates; in virtue of Corollary 5.6 the proof of Lemma 3.7 applies here too to show

5.7 Lemma. The pair $\left(\Delta_{f}(D), \hat{\varepsilon}\right)$ is a permutation symmetric specially directed semigroup of unital endomorphisms of $\mathfrak{A}_{D}$.

Since $\mathfrak{U}_{D}$ has trivial centre, Lemma 5.7 will allow us to use [14; Corollary 6.2]. This will be an intermediate step in establishing our key result, a variant of the spatial version of the cross product [14; Corollary 6.2] which applies to $\Delta_{f}$ without any reference to a choice of a particular double cone $D$.

If we want a completely general result we shall need to clarify one technical point of little practical importance. Should there be an uncountable number of sectors, the representation $\pi$ of the observable algebra in our field system will fail to be in the class $(S)$, i.e. to satisfy (1.2) simply because the Hilbert spaces $\mathscr{H}$ and $\mathscr{H}_{0}$ fail to have the same dimension. We therefore introduce the class $(\widetilde{S})$ of arbitrary direct sums of representations from $(S)$. If $\pi \in(\widetilde{S})$ we may still define $\pi \rho$ for any object $\rho$ of $\mathscr{T}_{t}$ and $\pi(T)$ for any arrow $T$ of $\mathscr{T}_{t}$ in the obvious manner: pick isometries $V_{i} \in\left(\pi_{i}, \pi\right)$ with $\pi_{i} \in(S)$ and $\sum_{i} V_{i} V_{i}^{*}=1_{\pi}$ and set

$$
\begin{aligned}
\pi \rho(A) & =\sum_{i} V_{i} \pi_{i} \rho(A) V_{i}^{*}, \\
\pi(T) & =\sum_{i} V_{i} \pi_{i}(T) V_{i}^{*} .
\end{aligned}
$$

These definitions are independent of the choice of the $V_{i}$ so that the results in Sect. 4 pertaining to $(S)$ hold trivially for $(\tilde{S})$ and this result will be used freely in the proof of Proposition 5.9 below. The following result, a simple consequence of Lemma 5.5, will also be used at several points in that proof.

5.8 Lemma. Let $\pi \in(\tilde{S})$, then $\pi$ admits a unique extension to a representation $\pi^{D}$ of $\mathfrak{U}_{D}$ which is normal in restriction to each $\mathfrak{U}(\mathscr{C})^{-}, D(\mathscr{C}) \subset D$. If $T$ is an arrow of $\mathscr{T}_{t}(D)$, then

$$
\pi^{D}(T)=\pi(T)
$$

If $\rho$ is an object of $\mathscr{T}_{t}(D)$ then

$$
(\pi \rho)^{D}=\pi^{D} \circ \rho^{D}
$$

Proof. $\pi$ is a direct sum of objects of $\mathscr{T}_{t}(D)$ so by Lemma $5.5 \pi$ obviously admits 
a unique extension to a representation $\pi^{D}$ of $\mathfrak{A}_{D}$ which is normal in restriction to each $\mathfrak{U}(\mathscr{C})^{-}, D(\mathscr{C}) \subset D$ and $\pi^{D}(T)=\pi(T)$ for each arrow $T$ of $\mathscr{T}_{t}(D)$. To prove the last part of the lemma, we pick isometries $V_{i} \in\left(\rho_{i}, \pi\right)$ with $\rho_{i} \in \Delta_{t}(D)$ and $\sum_{i} V_{i} V_{i}^{*}=1_{\pi}$. But by Lemma 4.5 we have $V_{i} \in\left(\rho_{i} \rho, \pi \rho\right)$. However we also have $V_{i} \in\left(\rho_{i}^{D}, \pi^{D}\right)$ and $V_{i} \in\left(\left(\rho_{i} \rho\right)^{D},(\pi \rho)^{D}\right)$ and $V_{i} \in\left(\rho_{i}^{D} \rho^{D}, \pi^{D} \circ \rho^{D}\right)$. Since $\left(\rho_{i} \rho\right)^{D}=\rho_{i}^{D} \rho^{D}$ by Lemma 5.5, we conclude that $(\pi \rho)^{D}=\pi^{D} \circ \rho^{D}$ as required.

5.9 Proposition. There is a Hilbert space $\mathscr{H}$ containing $\mathscr{H}_{0}$ as a subspace, a representation $\pi$ of $\mathfrak{U}$ on $\mathscr{H}$ in the class $(\widetilde{S})$, a (strongly) compact group $G$ of unitaries on $\mathscr{H}$ and a map $\rho \in \Delta_{f} \rightarrow H_{\rho}$ of $\Delta_{f}$ into the semigroup of Hilbert spaces with support $I$ in $\mathscr{B}(\mathscr{H})$, which is multiplicative on each $\Delta_{f}(\mathscr{C})$, such that
A) $\mathscr{H}_{0}=\{\Phi \in \mathscr{H}: g \Phi=\Phi, g \in G\}$;
B) $\pi_{0}$ is the restriction of $\pi$ to $\mathscr{H}_{0}$;
C) $\pi(\mathfrak{U}) \subset G^{\prime}$;
D) $\alpha_{g}\left(H_{\rho}\right) \equiv g H_{\rho} g^{-1}=H_{\rho} ; g \in G, \rho \in \Delta_{f}$;
E) $\psi \pi(A)=\pi \rho(A) \psi, \psi \in H_{\rho}, A \in \mathfrak{A}, \rho \in \Delta_{f}$;
F) $\theta\left(H_{\rho}, H_{\rho^{\prime}}\right)=\pi\left(\hat{\varepsilon}\left(\rho, \rho^{\prime}\right)\right), \rho, \rho^{\prime} \in \Delta_{f}(\mathscr{C}), \mathscr{C} \in \mathscr{J}$;
G) $\left(u_{\rho}, u_{\rho^{\prime}}\right)=\pi\left(\left(\rho, \rho^{\prime}\right)\right), \rho, \rho^{\prime} \in \Delta_{f}$;
H) the family $u_{\rho}, \rho \in \Delta_{f}$, separates the points of $G$.

The system $\left\{\pi, G, \rho \in \Delta_{f} \rightarrow H_{\rho}\right\}$ fulfilling $\left.\left.\mathrm{A}\right)-\mathrm{H}\right)$ is unique up to unitary equivalence. Namely, if $\left\{\pi_{1}, G_{1}, \rho \in \Delta_{f} \rightarrow H_{\rho}^{1}\right\}$ is another system acting on a Hilbert space $\mathscr{H}_{1}$, there is a unitary $W$ from $\mathscr{H}_{\text {to }} \mathscr{H}_{1}$ leaving $\mathscr{H}_{0}$ pointwise fixed such that

$$
\begin{aligned}
W \pi(A) & =\pi_{1}(A) W, & A \in \mathfrak{A}, \\
W G & =G_{1} W, & \\
W H_{\rho} & =H_{\rho}^{1} W, \quad & \rho \in \Delta_{f} .
\end{aligned}
$$

Proof. By Lemma 5.7 and [14; Corollary 6.2] there is a system

$$
\left\{\pi^{D}, G, \rho \in \Delta_{f}(D) \mapsto H_{\rho}\right\}
$$

acting on a Hilbert space $\mathscr{H} \supset \mathscr{H}_{0}$ and fulfilling the obvious variant of A)-H) of Sect. 3 for $\rho, \rho^{\prime} \in \Delta_{f}(D)$. By [14; Proposition 6.3]

$$
\pi^{D} \cong \bigoplus_{\xi} d(\xi) \pi_{0}^{D} \circ \rho_{\xi}^{D},
$$

where $\pi_{0}^{D}$ is the defining representation of $\mathfrak{U}_{D}$ on $\mathscr{H}_{0}$ and $\xi \mapsto \rho_{\xi}$ is a section of the unitary equivalence classes of all irreducible elements in $\Delta_{f}(D)$. If we let $\pi$ be the restriction of $\pi^{D}$ to $\mathfrak{A}$ then by (5.8),

$$
\pi \cong \bigoplus_{\xi} d(\xi) \pi_{0} \rho_{\xi}
$$

and $\pi$ belongs to the class $(\tilde{S})$. Hence $\pi(T)$ is defined by (4.5) and (5.6) for any arrow $T$ of $\mathscr{T}_{f}$ and (5.7) holds. If $\rho \in \Delta_{f}$ and $U \in\left(\rho_{1}, \rho\right)$ is unitary with $\rho_{1} \in \Delta_{f}(D)$, we set

$$
H_{\rho}:=\pi(U) H_{\rho_{1}}
$$

and check that this definition is independent of the choice of $U$. In fact, if $U^{\prime} \in\left(\rho_{1}^{\prime}, \rho\right)$ 
is unitary with $\rho_{1}^{\prime} \in \Delta_{f}(D)$, then by (5.7) and Lemma 4.4

$$
\pi^{D}\left(U^{*} \circ U^{\prime}\right)=\pi\left(U^{*} \circ U^{\prime}\right)=\pi(U)^{*} \pi\left(U^{\prime}\right)
$$

But by G) for the system (5.12), $\pi^{D}\left(U^{*} \circ U^{\prime}\right) H_{\rho_{1}^{\prime}}=H_{\rho_{1}}$ so $\pi\left(U^{\prime}\right) H_{\rho_{1}^{\prime}}=\pi(U) H_{\rho 1}$ as required. Conditions $\mathrm{A}$ ) and $\mathrm{C}$ ) are obviously fulfilled. B) follows since if $A \in \mathfrak{A}$,

$$
\left.\pi(A)\right|_{\mathscr{H}_{0}}=\left.\pi^{D}(A)\right|_{\mathscr{H} \mathrm{o}}=\pi_{0}^{D}(A)=\pi_{0}(A) .
$$

If $T$ is any arrow in $\mathscr{T}_{f}$ then

$$
\pi(T) \in G^{\prime}
$$

by (4.7). D) then holds for each $\rho \in \Delta_{f}$ as it already holds for $\rho \in \Delta_{f}(D)$. By E) for the system (5.12) and (5.3) we conclude that

$$
\psi \pi(A)=\pi \rho_{1}(A) \psi, \quad \psi \in H_{\rho_{1}}, \quad A \in \mathfrak{A}, \quad \rho_{1} \in \Delta_{f}(D) .
$$

Hence, by Lemma 4.6 and (5.15), E) holds. Since $\pi$ and $\pi \rho, \rho \in \Delta_{f}$ are in the class $(\tilde{S}),(4.7)$ shows that E) extends to give for each arrow $T$ of $\mathscr{T}_{f}$

$$
\psi \pi(T)=\pi \rho(T) \psi, \quad \psi \in H_{\rho}, \quad \rho \in \Delta_{f} .
$$

We turn now to the proof of F). Given $\rho, \rho^{\prime} \in \Delta_{f}(\mathscr{C})$ pick unitary intertwiners $U \in\left(\rho_{1}, \rho\right)$ and $U^{\prime} \in\left(\rho_{1}^{\prime}, \rho^{\prime}\right)$ with $\rho_{1}, \rho_{1}^{\prime} \in \Delta_{f}(D)$. Now bearing (5.15) in mind, the analogue of (3.15) for the permutation symmetry $\theta$ defined by (3.26) yields

$$
\theta\left(H_{\rho}, H_{\rho^{\prime}}\right) \pi(U) \pi \rho_{1}\left(U^{\prime}\right)=\pi\left(U^{\prime}\right) \pi \rho_{1}^{\prime}(U) \theta\left(H_{\rho_{1}}, H_{\rho_{1}^{\prime}}\right)
$$

after using (5.17). On the other hand, $\hat{\varepsilon}$ being a permutation symmetry, we have using Lemma 4.4,

$$
\pi\left(\hat{\varepsilon}\left(\rho, \rho^{\prime}\right)\right) \pi\left(U \times U^{\prime}\right)=\pi\left(U^{\prime} \times U\right) \pi\left(\hat{\varepsilon}\left(\rho_{1}, \rho_{1}^{\prime}\right)\right) .
$$

Furthermore, the remark following Theorem 4.10 shows that

$$
\pi\left(U \times U^{\prime}\right)=\pi(U) \pi \rho\left(U^{\prime}\right)
$$

so that F) now follows from the same property for the system (5.12) using (5.7). (5.15), (5.17) and (5.18) also show that $\rho \mapsto H_{\rho}$ is multiplicative on each $\Delta_{f}(\mathscr{C})$. To prove $G)$, we first note that by $(5.15)$ and $(5.16)$,

$$
\left(u_{\rho}, u_{\rho_{1}}\right) \pi(U)=\pi\left(U^{\prime}\right)\left(u_{\rho^{\prime}}, u_{\rho_{1}^{\prime}}\right) .
$$

On the other hand, by Lemma 4.4,

$$
\pi\left(\left(\rho, \rho^{\prime}\right)\right) \pi(U)=\pi\left(U^{\prime}\right) \pi\left(\left(\rho_{1}, \rho_{1}^{\prime}\right)\right) .
$$

Thus G) follows from the same property for the system (5.12) using (5.7) and (5.4). $\mathrm{H})$ is trivially true so the proof of existence is completed.

To prove uniqueness it will suffice to show that any system arises in the way just described. Given any such system since $\pi$ is in the class $(\widetilde{S})$ we can extend it to the representation $\pi^{D}$ of $\mathfrak{U}_{\boldsymbol{D}}$ using Lemma 5.8 and can restrict the map $\rho \mapsto H_{\rho}$ to $\Delta_{f}(D)$ to obtain a system (5.12) which trivially satisfies A), C) and D). $\pi_{0}^{D}$ is the restriction of $\pi^{D}$ to $\mathscr{H}_{0}$ since both are extensions of $\pi_{0}$ normal on each $\mathfrak{A}(\mathscr{C})^{-}$, $D(\mathscr{C}) \subset D$. Since $\pi \rho$ is in the class $(\widetilde{S})$ for $\rho \in \Delta_{f}(D)$, E) is a consequence of $(5.8)$ and 
the extension process of Lemma 5.8. F) is a consequence of (5.7) and G) follows from (5.7) and (5.4). $\mathrm{H}$ ) follows from $\mathrm{G}$ ) for the original system since for each $\rho \in \Delta_{f}$ there is a unitary $U \in\left(\rho_{1}, \rho\right)$ with $\rho_{1} \in \Delta_{f}(D)$. By the uniqueness statement in [14; Corollary 6.2] we can conclude that if $\left\{\pi_{1}, G_{1}, \rho \in \Delta_{f} \mapsto H_{\rho}^{1}\right\}$ is another system as described in the present proposition and acting on a Hilbert space $\mathscr{H}_{1} \supset \mathscr{H}_{0}$, there is a unitary $W$ from $\mathscr{H}$ to $\mathscr{H}_{1}$ leaving $\mathscr{H}_{0}$ pointwise fixed such that (5.9) and (5.10) hold and

$$
W H_{\rho}=H_{\rho}^{1} W, \quad \rho \in \Delta_{f}(D) .
$$

By (4.7), we automatically have $W \pi(T)=\pi_{1}(T) W$ for an arrow $T$ of $\mathscr{T}_{f}$ so that by G) using a unitary intertwiner we may pass from (5.19) to (5.11) completing the proof.

Having established Proposition 5.9, the proof of the existence of a complete, normal extended field system with gauge symmetry proceeds as in Sect. 3 and we will not bother to repeat those arguments which apply without major changes.

To prove the existence claim of Theorem 5.3, we now define

$$
\mathfrak{F}(\mathscr{C})=\left\{H_{\rho}, H_{\rho}^{*} ; \rho \in \Delta_{f}(\mathscr{C})\right\}^{\prime \prime} .
$$

For each double cone $D$ at spacelike infinity, the $C^{*}$-algebra generated by $\pi^{D}\left(\mathfrak{A}_{D}\right)$ and by $\left\{H_{\rho} ; \rho \in \Delta_{f}(D)\right\}$ is irreducible by [14; Proposition 6.4]. Since every unitary $U \in \mathfrak{A}(\mathscr{C})^{-}$, with $D(\mathscr{C}) \subset D$, provides a Hilbert space $\mathbf{C} \cdot \pi(U)$ in the family $\left\{H_{\rho} ; \rho \in \Delta_{f}(D)\right\}, \pi(\mathfrak{U}(\mathscr{C}))^{-} \subset \mathfrak{F}(\mathscr{C})$ and

$$
\mathfrak{F}_{D}^{\prime \prime}=\left\{H_{\rho}, H_{\rho}^{*} ; \rho \in \Delta_{f}(D)\right\}^{\prime \prime}=\mathscr{B}(\mathscr{H}) .
$$

The arguments given in Sect. 3 now apply to show that $\{\pi, G, \mathfrak{F}\}$ is a complete extended field system with gauge symmetry. To prove that it is also normal, along the lines followed there, it suffices to show that, if $\rho, \rho^{\prime} \in \Delta_{f}$ are irreducible and localized in $\mathscr{C}_{1}$ and $\mathscr{C}_{2}$ respectively, where $\mathscr{C}_{1} \subset \mathscr{C}_{2}^{\prime}$, then

$$
\psi \psi^{\prime}= \pm \psi^{\prime} \psi, \quad \psi \in H_{\rho}, \quad \psi^{\prime} \in H_{\rho^{\prime}}
$$

where the minus sign applies only if both $\rho$ and $\rho^{\prime}$ are para-Fermi.

If there is a common spacelike cone $\mathscr{C}$ containing $\mathscr{C}_{1}, \mathscr{C}_{2}$, then (5.21) follows, as in Sect. 3, from F) in Proposition 5.9. In the general case, we can choose spacelike cones $\mathscr{C}_{0 i} \subset \mathscr{C}_{i}$ and $\mathscr{C}$ with $\mathscr{C}_{0 i} \subset \mathscr{C}, i=1,2$ (cf. Appendix h)). By choosing unitary intertwiners $U \in\left(\rho, \rho_{1}\right), U^{\prime} \in\left(\rho^{\prime}, \rho_{2}\right), \rho_{i} \in \Delta_{f}\left(\mathscr{C}_{0 i}\right)$, we have by relation (5.15) and by F) that

$$
\begin{aligned}
\psi & =\pi(U) \psi_{1}, \psi_{1} \in H_{\rho_{1}}, \\
\psi^{\prime} & =\pi\left(U^{\prime}\right) \psi_{2}, \psi_{2} \in H_{\rho_{2}}, \\
\psi_{1} \psi_{2} & = \pm \psi_{2} \psi_{1} .
\end{aligned}
$$

By our choice of $U$ and $U^{\prime}$, we have $U \in \mathfrak{A}\left(\mathscr{C}_{1}\right)^{-}, U^{\prime} \in \mathfrak{A}\left(\mathscr{C}_{2}\right)^{-}$and $\rho_{1}\left(U^{\prime}\right)=U^{\prime}$, $\rho_{2}(U)=U$. Thus (5.21) now follows from (5.22) and from E), and the proof of existence is complete.

One has to go about proving uniqueness in the same way as in Sect. 3, first proving Theorem 5.4 and then using it to show that any complete, normal extended 
field system with gauge symmetry is determined as above by the data described in Proposition 5.9. The uniqueness assertion of Proposition 5.9 implies that there is, up to equivalence, a unique complete normal extended field system with gauge symmetry.

Now the relevant arguments of Sect. 3 are essentially algebraic in nature and hence easily adapted to the different geometrical context of this section. We will therefore refrain from repeating them and content ourselves with a brief sketch.

Let $\{\pi, G, \mathfrak{F}\}$ be an extended field system with gauge symmetry. Then, by $\gamma$ ) and $\delta$ ), $\pi \mid \mathfrak{A}(\mathscr{C})$ is quasiequivalent to $\pi_{0} \mid \mathfrak{A}(\mathscr{C})$ (cf. Lemma 3.11). Hence (cf. Lemma 3.8) $\mathscr{H}_{0}$ separates $\mathfrak{F}_{D}$ and consists precisely of all $G$-invariant vectors; also

$$
\begin{gathered}
\pi(\mathfrak{U})^{\prime}=G^{\prime \prime}, \\
\mathfrak{F}(\mathscr{C})^{\prime} \cap G^{\prime}=\pi\left(\mathfrak{U}\left(\mathscr{C}^{\prime}\right)\right)^{-}, \quad \mathscr{C} \in \mathscr{J} .
\end{gathered}
$$

The arguments of Sect. 3 go through unchanged since $\mathfrak{F}_{D}$ is generated by a net and is irreducible by $\beta$ ) of Definition 5.1. As already indicated, $\mathscr{H}_{0}$ cannot be expected to be separating for the $C^{*}$-algebra generated by all $\mathfrak{F}(\mathscr{C})$.

By (5.23) the classes $\xi$ of irreducible representations appearing in the decomposition of the defining representation of $G$ label the classes of irreducible subrepresentations of $\pi$. If $\pi_{\xi}$ is such a subrepresentation, we can prove as in Sect. 3 (Lemma 3.11) that

$$
\pi_{\xi}\left|\mathfrak{U}\left(\mathscr{C}^{\prime}\right) \cong \pi_{0}\right| \mathfrak{U}\left(\mathscr{C}^{\prime}\right), \quad \mathscr{C} \in \mathscr{J} .
$$

Here too this is the only place where the separability of $\mathscr{H}_{0}$ enters.

Of course the reason why the arguments of Sect. 3 do not give more in the present case, that is why (3.33) itself might fail, is that we do not now have local algebras of field operators cyclic on $\mathscr{H}_{0}$ (cf. the comments below).

If $\rho \in \Delta_{t}(D)$ we now use $\mathfrak{F}_{D}$ to locate the Hilbert space inducing $\rho$ by setting

$$
H_{\rho} \equiv\left\{\psi \in \mathfrak{F}_{D}: \psi \pi(A)=\pi \rho(A) \psi, A \in \mathfrak{U}\right\} .
$$

Since $\pi(\mathfrak{U})^{\prime}=G^{\prime \prime}$ and $\mathscr{H}_{0}$ separates $\mathfrak{F}_{D}$, we have $\pi(\mathfrak{U})^{\prime} \cap \mathfrak{F}_{D}=\mathbf{C} \cdot I$ and $H_{\rho}$ is indeed a $G$-stable Hilbert space. We can prove as in Sect. 3 that, if $\rho \in \Delta_{t}(D)$ is an irreducible subrepresentation of $\pi, H_{\rho}$ has support $I$. Next we show that $H_{\rho}$ is independent of $D$, i.e., denoting it temporarily by $H_{\rho}^{D}, H_{\rho}^{D_{1}}=H_{\rho}^{D_{2}}$ if $\rho \in \Delta_{t}\left(D_{1}\right) \cap \Delta_{t}\left(D_{2}\right)$. This is clear if $D_{1} \subset D_{2}$ since $H_{\rho}^{D_{1}} \subset H_{\rho}^{D_{2}}$ and a Hilbert space of support $I$ is maximal. In general there are, by g) of the Appendix, double cones at spacelike infinity $D$ and $D_{0 i} \subset D_{i} \cap D, i=1,2$. Choosing unitary intertwiners

$$
\begin{array}{ll}
U_{i} \in\left(\rho_{0 i}, \rho\right), & \rho_{0 i} \in \Delta_{t}\left(D_{0 i}\right), \\
U_{i}^{\prime} \in\left(\rho^{\prime}, \rho_{0 i}\right), & \rho^{\prime} \in \Delta_{t}(D), \quad i=1,2,
\end{array}
$$

we have by the last remark that $H_{\rho_{0_{i}}}^{D 0_{i}}=H_{\rho_{0_{i}}}^{D_{i}}=H_{\rho_{0_{i}}}^{D} \equiv H_{\rho_{0_{i}}}$ and also that, using (5.26)

Then, by Lemma 4.4,

$$
\begin{aligned}
& H_{\rho}^{D_{i}}=\pi\left(U_{i}\right) H_{\rho_{0_{i}}} \\
& H_{\rho_{0_{i}}}=\pi\left(U_{i}^{\prime}\right) H_{\rho^{\prime}}^{D}, \quad i=1,2 .
\end{aligned}
$$

$$
H_{\rho}^{D_{\imath}}=\pi\left(U_{i}^{\circ} U_{i}^{\prime}\right) H_{\rho^{\prime}}^{D}
$$


and

$$
H_{\rho}^{D_{2}}=\pi(V) H_{\rho}^{D_{1}}
$$

with

$$
V \equiv U_{2} \circ U_{2}^{\prime} \circ\left(U_{1} \circ U_{1}^{\prime}\right)^{*} \in(\rho, \rho) .
$$

Since $V$ is unitary we get $\pi(V) H_{\rho}^{D_{1}}=H_{\rho}^{D_{1}}$ and $H_{\rho}^{D_{1}}=H_{\rho}^{D_{2}}$.

Lemma 3.13 is valid here too if we read $\mathscr{C} \in \mathscr{J}$ for $\mathcal{O} \in \mathscr{K}$. Similarly, Theorem 3.14 holds with just the same change if we again define

$$
\Delta_{\mathfrak{F}}=\left\{\rho \in \Delta_{t}: H_{\rho} \text { is a finite dimensional Hilbert space with support } I\right\},
$$

where $H_{\rho}$ is now defined by (5.26) for each $\rho \in \Delta_{t}$, and $\Delta_{f}$ plays the role of $\Delta$ in the statement of Theorem 3.14. In contrast to the footnote appended there, $\mathscr{T}_{\mathscr{F}}$ is not itself a monoidal category, but only $\mathscr{T}_{\mathscr{F}} \cap \mathscr{T}_{D}$, for each double cone $D$ at spacelike infinity.

At this point we can proceed as in Sect. 3 to show that if $\{\pi, G, \mathfrak{F}\}$ is a normal extended field system with gauge symmetry, then

$$
\begin{gathered}
\theta\left(H_{\rho}, H_{\rho^{\prime}}\right)=\pi\left(\hat{\varepsilon}\left(\rho, \rho^{\prime}\right)\right), \quad \rho, \rho^{\prime} \in \Delta_{f} \cap \Delta(\mathscr{C}), \quad \mathscr{C} \in \mathscr{J} ; \\
\mathscr{F}^{t}(\mathscr{C})=\mathfrak{F}\left(\mathscr{C}^{\prime}\right)^{\prime}, \quad \mathscr{C} \in \mathscr{J} ;
\end{gathered}
$$

where the twisting is defined as in Eq. (3.8) but for spacelike cones.

The uniqueness statement in Proposition 5.9 can now be applied to give uniqueness in Theorem 5.3.

We could here too discuss abnormal commutation relations and subsystems, as well as the additivity property for spacelike cones, along the lines which led to Theorem 3.15, Propositions 3.16-18.

We limit ourselves to a comment on a subsystem of special relevance. Given the complete normal extended field system with gauge symmetry $\{\pi, G, \mathfrak{F}\}$, we can define the subspace $\mathscr{H}_{l}$ of $\mathscr{H}$ reached by localized fields acting on the vacuum by

$$
\begin{aligned}
\mathscr{H}_{l} & =\left\{H_{\rho}, H_{\rho}^{*} ; \rho \in \Delta\right\}^{\prime \prime} \mathscr{H}_{0} \\
& =\left\{H_{\rho}, H_{\rho}^{*} ; \rho \in \Delta(\mathcal{O})\right\}^{\prime \prime} \mathscr{H}_{0}, \quad \mathcal{O} \in \mathscr{K} ;
\end{aligned}
$$

and we can define a field system on $\mathscr{H}_{l}$ by

$$
\mathfrak{F}_{l}(\mathcal{O})=\left.\left\{H_{\rho}, H_{\rho}^{*} ; \rho \in \Delta(\mathcal{O})\right\}^{\prime \prime}\right|_{\mathscr{H}_{l}} .
$$

Then $\mathscr{H}_{l}$ is obviously stable under $G$ and $\pi(\mathfrak{A})$ and we can set

$$
\pi_{l}=\left.\pi\right|_{\mathscr{H}_{l}} ; \quad G_{l}=\left.G\right|_{\mathscr{H}_{l}} .
$$

The system $\left\{\pi_{l}, G_{l}, \mathcal{O} \rightarrow \mathfrak{F}_{l}(\mathcal{O})\right\}$ is just the complete normal (local) field system with gauge symmetry given by Theorem 3.5. Defining

$$
\mathfrak{F}_{l}(\mathscr{C})=\left\{\mathfrak{F}_{l}(\mathcal{O}), \mathcal{O} \subset \mathscr{C}, \mathcal{O} \in \mathscr{K}\right\}^{\prime \prime}
$$

we would get the subsystem defined, as in Proposition 3.16, by the closed normal subgroup $H \subset G$, which is the kernel of the restriction map $\left.g \in G \rightarrow g\right|_{\mathscr{H}_{l}}$ and $G_{l}=G / H$. 
Therefore a superselection sector $\xi$ carries a topological charge if and only if the irreducible representation of $G$ it corresponds to is non-trivial on $H$.

The homomorphism $\left.g \in G \rightarrow g\right|_{\mathscr{F}_{l}} \in G_{l}$ can also be viewed as corresponding to the embedding of $\mathscr{T}$ into $\mathscr{T}_{f}(D)$ by Theorem 6.10 of [2].

Starting from a local field system we can always define an extended field system by setting $\mathfrak{F}(\mathscr{C}) \equiv\{\mathfrak{F}(\mathcal{O}): \mathcal{O} \in \mathscr{K}, \mathcal{O} \subset \mathscr{C}\}^{\prime \prime}$ (it will, in general, not be complete even if the original local system were complete). We might ask whether we can go the other way around and define a local system from an extended system by first setting

$$
\mathfrak{F}(\mathcal{O}) \equiv \bigcap_{\mathscr{C} \supset \mathcal{O}} \mathfrak{F}(\mathscr{C})
$$

The trouble is that, at the present level of generality, it is not guaranteed that $\overline{\mathfrak{F}(\mathcal{O}) \mathscr{H}_{0}}=\overline{\bigcup \mathscr{F}(\mathcal{O}) \mathscr{H}_{0}}$ which would be a familiar consequence of the ReehSchlieder theorem if the net (5.32) satisfied additivity.

However by using further standard assumptions of Quantum Field Theory we can conclude that

$$
\begin{aligned}
\overline{\mathfrak{F}(\mathcal{O}) \mathscr{H}_{0}} & =\mathscr{H}_{l}, \\
\mathfrak{F}_{l}(\mathcal{O}) & =\left.\mathfrak{F}(\mathcal{O})\right|_{\mathscr{H}_{l}}, \\
\mathfrak{F}(\mathcal{O}) & =\left\{H_{\rho}, H_{\rho}^{*} ; \rho \in \Delta(\mathcal{O})\right\}^{\prime \prime}, \quad \mathcal{O} \in \mathscr{K} .
\end{aligned}
$$

Namely we assume now that the net $\mathfrak{A}(\mathcal{O})$ fulfills the additivity property $\mathfrak{U}(\mathcal{O}) \subset\left\{\bigcup_{i=1}^{n} \mathfrak{U}\left(\mathcal{O}_{i}\right)\right\}^{\prime \prime}$ if $\mathcal{O} \subset \bigcup_{i=1}^{n} \mathcal{O}_{i}, \mathcal{O}, \mathcal{O}_{1}, \ldots, \mathcal{O}_{n} \in \mathscr{K}$, and that the representation $\pi$ is translation covariant and fulfills the spectrum condition (cf. Sect. 6). Moreover, this is the unique point of our discussion where the restrictive nature of condition (1.1) reveals itself. We required (1.1) to hold for all double cones, but this might well be a consequence of its validity for all the translates of a given double cone. We will assume now that this is the case, just to avoid reformulating all our results, since the physical relevance of such a generalization would be doubtful.

Under the stated assumptions, to conclude that a sector $\pi_{\xi}$ appearing in the representation $\pi$ is associated with a localizable charge, it suffices, by the arguments in [1; Appendix], that, for some $\mathcal{O} \in \mathscr{K}$, the representations $\pi_{\xi}, \pi_{0}$ restricted to $\mathfrak{A}\left(\mathcal{O}^{\prime}\right)$ are not disjoint:

$$
\pi_{0}\left|\mathfrak{U}\left(\mathcal{O}^{\prime}\right){ }^{\dagger} \pi_{\xi}\right| \mathfrak{U}\left(\mathcal{O}^{\prime}\right) \text {. }
$$

With this information the statements (5.33), (5.34), (5.35) are easily proved. Indeed we need only show that

$$
\mathfrak{F}(\mathcal{O}) \subset\left\{H_{\rho}, H_{\rho}^{*}, \rho \in \Delta(\mathcal{O})\right\}^{\prime \prime}, \quad \mathcal{O} \in \mathscr{K},
$$

since the opposite inclusion is trivial by (5.32), and the other relations follow easily. If $X_{1}, \ldots, X_{n} \in \mathfrak{F}(\mathcal{O})$ is an irreducible tensor under $G$, we have to show that the corresponding sector carries a localizable charge $\xi$. There would then be a $\rho \in \Delta(\mathcal{O})$ such that an orthonormal basis $\psi_{1}, \ldots, \psi_{n}$ in $H_{\rho}$ transforms like $X_{1}, \ldots, X_{n}$ under $G$. Hence we would have $X_{i}=\pi(B) \psi_{i}$, where $\pi(B)=\Sigma_{i} X_{i} \psi_{i}^{*}$ is in $\pi(\mathfrak{A}(\mathcal{O}))$ by duality in the vacuum sector. Then $X_{1}, \ldots, X_{n} \in\left\{H_{\rho}, H_{\rho}^{*} ; \rho \in \Delta(\mathcal{O})\right\}^{\prime \prime}$ as required. 
Let $E_{0}, E_{\xi}$ be the orthogonal projections of $\mathscr{H}$ onto $\mathscr{H}_{0}$ and $\mathscr{H}_{\xi}$ respectively, where $\mathscr{H}_{\xi}$ is an irreducible subspace for $\pi$ carrying a subrepresentation of the class $\xi$. Then for some $i E_{\xi} X_{i}^{*} E_{0}$ is nonzero and, by locality of fields relative to the observables, it intertwines the restrictions to $\mathfrak{A}\left(\mathcal{O}^{\prime}\right)$ of the subrepresentations of $\pi$ on $\mathscr{H}_{0}$ and $\mathscr{H}_{\xi}$. Hence (5.36) holds and the inclusion $\left(5.35^{\prime}\right)$ is verified.

Note that the above conclusion allows us to define $H$ more directly: it is the kernel of

$$
g \in G \rightarrow \alpha_{g} \mid \mathfrak{F}\left(\mathscr{C}_{1}\right) \cap \mathfrak{F}\left(\mathscr{C}_{2}\right)
$$

where $\mathscr{C}_{1}, \mathscr{C}_{2}$ are fixed spacelike cones with nonempty intersection such that $\mathscr{C}_{1}^{\prime} \cup \mathscr{C}_{2}^{\prime} \supset \mathcal{O}^{\prime}$ for some double cone $\mathcal{O}$. For the additivity property of $\mathfrak{A}$ then implies

$$
\left(\pi\left(\mathfrak{A}\left(\mathscr{C}_{1}^{\prime}\right)\right) \cup \pi\left(\mathfrak{A}\left(\mathscr{C}_{2}^{\prime}\right)\right)\right)^{\prime} \subset \pi\left(\mathfrak{A}\left(\mathcal{O}^{\prime}\right)\right)^{\prime}
$$

and if $\mathscr{O}_{1} \in \mathscr{K}, \mathcal{O}_{1} \subset \mathscr{C}_{1} \cap \mathscr{C}_{2}$, we have

$$
\mathfrak{F}\left(\mathcal{O}_{1}\right) \subset \mathfrak{F}\left(\mathscr{C}_{1}\right) \cap \mathfrak{F}\left(\mathscr{C}_{2}\right) \subset \mathfrak{F}(\mathcal{O}),
$$

where the first inclusion is obvious and the second one follows from the above argument. Since $H$ is the kernel of $\left.g \in G \rightarrow \alpha_{g}\right|_{\mathscr{F}(\mathcal{O})}$ for any $\mathcal{O}$, the assertion follows.

The reader will have noticed that one of the added difficulties when dealing with charges localized in spacelike cones was that our category $\mathscr{T}_{f}$ was not embedded as a full subcategory of End $\widetilde{\mathfrak{A}}$ for some $C^{*}$-algebra $\widetilde{\mathfrak{A}}$. We end this section with some comments on why there is no natural choice of $\tilde{\mathfrak{A}}$.

One might be tempted to use the $C^{*}$-algebras $\tilde{\mathscr{A}}$ and $\tilde{\mathscr{F}}$ generated by all $\pi(\mathfrak{H}(\mathscr{C}))^{-}$ and $\mathscr{F}(\mathscr{C})$ respectively, as $\mathscr{C}$ varies over the spacelike cones. However this $C^{*}$-algebras may now contain global charge operators. Actually it may be expected that, in contrast to the simplicity of $\mathfrak{A}[18]$ and to Theorem 5.4 a), in typical cases we have

$$
\begin{aligned}
\tilde{\mathfrak{A}} \cap \tilde{\mathfrak{A}}{ }^{\prime} & =\pi(\mathfrak{A})^{\prime \prime} \cap \pi(\mathfrak{A})^{\prime}=G^{\prime} \cap G^{\prime \prime}, \\
\pi(\mathfrak{A})^{\prime} & =G^{\prime \prime} \subset \tilde{\mathbb{F}} .
\end{aligned}
$$

These conclusions are suggested if we assume the split property for the net $\mathfrak{F}$, i.e. the inclusion $\mathscr{F}\left(\mathscr{C}_{1}\right) \subset \mathscr{F}\left(\mathscr{C}_{2}\right)$ is split whenever $\overline{\mathscr{C}}_{1} \subset \mathscr{C}_{2}$ and $\overline{D\left(\mathscr{C}_{1}\right)} \subset D\left(\mathscr{C}_{2}\right)$. We would then have local implementations of the gauge group on cones [26] and additivity properties may be expected for the corresponding local generators and local charge operators, so that the global ones would be a finite sum of the local ones. This motivates the conjecture expressed by (5.37), (5.38).

These equations would show in particular that $\tilde{\mathfrak{A}}$ is not faithfully represented in the different sectors, a fact that could be expected on intuitive grounds. For the algebraic equivalence of different sectors relies on the possibility of compensating any charge distribution with corresponding conjugate charges localized far away [4]. If, however, by taking all weak limit points of observables within each spacelike cone, we allow global observables which measure the distribution of charges on double cones at spacelike infinity, this compensation is no longer possible (cf. Appendix, c)). Indeed, the intersection of spacelike infinity with any spacelike hyperplane is a compact manifold and there is no room to localize charges "far away." 


\section{Covariance and Spectrum Condition}

We derived our main result, Theorem 5.3, assuming only a few general properties, of an algebraic nature, about the vacuum sector, which in fact do not suffice to characterize $\pi_{0}$ as a vacuum representation. Indeed, for each $\rho \in \Delta_{f}$ with $d(\rho)=1$, $\pi_{0} \rho$ would enjoy the same properties.

In physical terms, a vacuum state can be singled out as being a ground state in its own representation. As is well known, this means, more precisely, that we have a strongly continuous unitary representation $\mathscr{U}_{0}$ of the spacetime translation group acting on $\mathscr{H}_{0}$ such that

$$
\begin{gathered}
\mathscr{U}_{0}(a) \mathfrak{H}(\mathcal{O}) \mathscr{U}_{0}(a)^{-1}=\mathfrak{A}(\mathcal{O}+a), \quad a \in \mathbf{R}^{4}, \quad \mathcal{O} \in \mathscr{K}, \\
\text { Spectrum } \mathscr{U}_{0} \subset \overline{V^{+},}
\end{gathered}
$$

and a unit vector $\Omega \in \mathscr{H}_{0}$ (the vacuum state vector) invariant under $\mathscr{U}_{0}$,

$$
\mathscr{U}_{0}(a) \Omega=\Omega, \quad a \in \mathbf{R}^{4} .
$$

The translation covariance of the theory is expressed by the action $\alpha$ of $\mathbf{R}^{4}$ by automorphisms of $\mathfrak{A}$ induced by $\mathscr{U}_{0}$,

$$
\alpha_{a}(A)=\mathscr{U}_{0}(a) A \mathscr{U}_{0}(a)^{-1}, \quad a \in \mathbf{R}^{4}, \quad A \in \mathfrak{A}
$$

and $\left(\pi_{0}, \mathscr{U}_{0}\right)$ can be thought of as a covariant representation of $\{\mathfrak{U}, \alpha\}$.

Superselection sectors are also expected to have the property that the energy can be defined and is bounded below; more precisely they should correspond to covariant representations $\left(\pi_{\xi}, \mathscr{U}_{\xi}\right)$ of $\{\mathfrak{A}, \alpha\}$ fulfilling the spectrum condition

$$
\begin{gathered}
\mathscr{U}_{\xi}(a) \pi_{\xi}(A) \mathscr{U}_{\xi}(a)^{-1}=\pi_{\xi} \circ \alpha_{a}(A) \quad a \in \mathbf{R}^{4}, \quad A \in \mathfrak{A}, \\
\text { Spectrum } \mathscr{U}_{\xi} \subset \overline{V^{+}} .
\end{gathered}
$$

These conditions, strengthened to require $\pi_{\xi}$ to be a massive particle representation, have been taken as the starting point for the analysis of superselection structure by Buchholz and Fredenhagen, who then derived property (1.2) for $\pi_{\xi}$ relative to some vacuum representation [6].

In our approach, localization properties relative to a given vacuum are emphasized and it would be desirable to deduce the spectrum condition (6.5), (6.6) for our sectors from localization properties (1.2), finite statistics, and from fundamental properties of the theory such as the spectrum condition in the vacuum sector (6.1)-(6.3).

This problem however falls outside the scope of this paper and we will rather restrict attention to the covariant elements in $\Delta_{f}$ leaving open the possibility that in sensible theories each element of $\Delta_{f}$ might be automatically covariant.

First we discuss the simple case where full Poincare covariance is assumed in the vacuum sector and we focus attention on Poincaré covariant localized morphisms.

More precisely, let $\mathscr{U}_{0}$ denote the strongly continuous unitary representation of the covering group $\widetilde{\mathscr{P}}$ of the Poincare group $\mathscr{P}$ which describes the covariance 
of the vacuum sector, so that the analogues of Eqs. (6.1)-(6.3) hold ${ }^{32}$. Let $\alpha$ denote the action of $\widetilde{P}$ on $\mathfrak{U}$ induced by $\mathscr{U}_{0}$. We consider those $\rho \in \Delta$ for which there is a covariant representation $\left(\rho, \mathscr{U}_{\rho}\right)$ of $\{\mathfrak{U}, \alpha\}$.

As was discussed in [9; Sects. 2,5], the covariant elements in $\Delta$ form a subset which is stable under products, subrepresentations, direct sums and conjugates. By Theorem 3.15 there is then a unique corresponding normal field system with gauge symmetry.

The results in $[9$, Sects. 2,5$]$ also show that $\mathscr{U}_{\rho}$ is unique, it fulfills the spectrum condition and the $\left(\rho, \mathscr{U}_{\rho}\right)$ form a coherently covariant family of representations in the sense of [14; Theorem 8.4] (cf. Eqs. (2.3), (2.5), (2.6), (2.8), (2.9) and Lemma 2.2 of [9]).

From these facts it is easy to adapt Theorem 8.4 of [14] to the construction of Sect. 3 above, to obtain

6.1 Theorem. Let $\{\pi, G, \mathfrak{F}\}$ denote the unique normal field system with gauge symmetry such that an irreducible representation of $\mathfrak{A}$ fulfilling (1.2) with finite statistics is contained in $\pi$ if and only if it is covariant for the action $\alpha$ of the covering group $\tilde{\mathscr{P}}$ of the Poincaré group on $\mathfrak{A}$.

There is then a unique strongly continuous unitary representation $\mathscr{U}$ of $\tilde{\mathscr{P}}$ on $\mathscr{H}_{\pi}$ such that $(\pi, \mathscr{U})$ is a covariant representation of $\{\mathfrak{U}, \alpha\}$ and this $\mathscr{U}$ satisfies:

(i) $\mathscr{U}(L) \Omega=\Omega, \quad L \in \tilde{\mathscr{P}}$;

(ii) Spectrum $\mathscr{U} \mid \mathbf{R}^{4} \subset \overline{V^{+}}$;

(iii) $\mathscr{U}(\tilde{\mathscr{P}}) \subset \pi(\mathfrak{U})^{\prime \prime}$;

(iv) $\mathscr{U}(L) \mathfrak{F}(\mathcal{O}) \mathscr{U}(L)^{-1}=\mathfrak{F}(L \mathcal{O}), \quad L \in \tilde{P}, \quad \mathcal{O} \in \mathscr{K}$.

We will refrain from giving a proof of this theorem here since the quoted references and Theorem 6.3 discussed in detail below should provide a sufficient guide.

We now turn to the analysis of sectors which are only translation covariant and describe both localized and topological charges. The vacuum representation is assumed to be translation covariant and to fulfill the spectrum condition (6.1), (6.2), (6.3), and $\alpha$ will denote the action of translations on $\mathfrak{A}$ given by (6.4).

We will consider the subset $\Delta_{c}$ consisting of all $\rho \in \Delta_{f}$ for which there is a covariant representation $\left(\rho, \mathscr{U}_{\rho}\right)$ of $\{\mathfrak{U}, \alpha\}$. Since $\rho(\mathfrak{U})^{\prime}$ is finite dimensional by Theorem 4.13 , the action induced by $\mathscr{U}_{\rho}$ on $\rho(\mathfrak{U})^{\prime}$ is uniformly continuous. Hence by [27, Theorem 8.5.2] this action is inner and there is a covariant representation $\left(\rho, \mathscr{U}_{\rho}\right)$ such that $\mathscr{U}_{\rho}(a) \in \rho(\mathfrak{U})^{\prime \prime}, a \in \mathbf{R}^{4}$.

Therefore each subrepresentation of $\rho$ is also covariant and $\Delta_{c}$ is closed under subobjects and direct sums.

Moreover $\Delta_{c}$ is a subsemigroup of $\Delta_{f}$ : if $\left(\rho, \mathscr{U}_{\rho}\right)$ and $\left(\rho^{\prime}, \mathscr{U}_{\rho^{\prime}}\right)$ are covariant representations of $\{\mathfrak{A}, \alpha\}$, where $\rho, \rho^{\prime} \in \Delta_{c}$, define

then

$$
W_{\rho}(a)=\mathscr{U}_{0}(a) \mathscr{U}_{\rho}(a)^{-1}, \quad a \in \mathbf{R}^{4},
$$

$$
W_{\rho}(a) \in\left(\rho, \alpha_{a} \rho \alpha_{a}^{-1}\right)
$$

$32 \mathscr{U}_{0}$ of course factors through $\mathscr{P}$ but $\mathscr{U}_{\rho}$, in general, does not 
is a strongly continuous 1 -cocycle for $\{\mathfrak{U}, \alpha\}$. It is easy to check that $\left(\rho \rho^{\prime}, \mathscr{U}_{\rho \rho^{\prime}}\right)$ is a covariant representation of $\{\mathfrak{U}, \alpha\}$, where

$$
\mathscr{U}_{\rho \rho^{\prime}}(a)=\rho\left(W_{\rho^{\prime}}(a)\right)^{-1} \mathscr{U}_{\rho}(a), \quad a \in \mathbf{R}^{4} .
$$

We next note that $\Delta_{c}$ is stable under conjugates and that, if $\rho \in \Delta_{c}, \mathscr{U}_{\rho}$ can also be chosen to fulfill the spectrum condition. Obviously it suffices to verify these assertions for the irreducible elements.

If $\left(\rho, \mathscr{U}_{\rho}\right)$ is a covariant representation of $\{\mathfrak{U}, \alpha\}$ with $\rho$ an irreducible element of $\Delta_{c}$ and $\bar{\rho}$ is a conjugate for $\rho$, the arguments leading to Theorem 7.1 of [6] and Theorem 5.2 of [9] show that there also exists a strongly continuous unitary representation $\mathscr{U}_{\bar{\rho}}$ of the translation group such that $\left(\bar{\rho}, \mathscr{U}_{\bar{\rho}}\right)$ is covariant and that

Spectrum $\mathscr{U}_{\rho}+$ Spectrum $\mathscr{U}_{\bar{\rho}} \subset$ Spectrum $\mathscr{U}_{0}$.

Therefore, since Spectrum $\mathscr{U}_{0} \subset \bar{V}_{+}$, multiplying $\mathscr{U}_{\rho}$ by a character if necessary, we conclude that we can choose $\mathscr{U}_{\rho}$ such that

$$
\text { Spectrum } \mathscr{U}_{\rho} \subset \bar{V}_{+} .
$$

In the case of a Poincaré covariant theory, the uniqueness of the representation $\mathscr{U}$ of $\tilde{\mathscr{P}}$ making $(\pi, \mathscr{U})$ covariant, where $\pi(\mathfrak{U})^{\prime}$ is a direct sum of finite dimensional full matrix algebras, was a simple consequence of the fact that $\tilde{P}$ does not have non-trivial finite dimensional unitary representations. In the case of translation covariance alone, we have to use both the spectrum condition and the local structure of $\mathfrak{U}$ to get a unique choice of $\mathscr{U}$. A covariant representation $(\pi, \mathscr{U})$ of $\{\mathfrak{U}, \alpha\}$ is said to be minimal if

(i) Spectrum $\mathscr{U} \subset \bar{V}^{+}$;

(ii) $\mathscr{U}(a) \in \pi(\mathfrak{U})^{\prime \prime}, a \in \mathbf{R}^{4}$;

(iii) for each projection $E \in \pi(\mathfrak{U})^{\prime}$ and $p \in \bar{V}^{+}$, Spectrum $\mathscr{U} \mid E \mathscr{H} \subset p+\bar{V}^{+} \Rightarrow p=0$.

This notion has been introduced in [28] (cf. also [6]) where it is proved that, if $(\pi, \mathscr{U})$ is a covariant representation fulfilling (i), there is a unique choice of $\mathscr{U}$ such that $(\pi, \mathscr{U})$ is minimal covariant. The key point is that the spectrum of this $\mathscr{U}$ has Lorentz invariant lower boundary; more precisely it must contain the full orbit of mass $m,\left\{p: p^{2}=m^{2}, p_{0}>0\right\}$, where $m$ is the minimum of the spectrum of the associated mass operator in the orthogonal complement of the $\mathscr{U}$-invariant vectors.

For each $\rho \in \Delta_{c}$, let $\mathscr{U}_{\rho}$ denote the unique representation such that $\left(\rho, \mathscr{U}_{\rho}\right)$ is minimal covariant, and let $W_{\rho}$ be the associated cocycle defined by (6.7). The main properties of the representations $\left(\rho, \mathscr{U}_{\rho}\right), \rho \in \Delta_{c}$, are summarized in the following

6.2 Proposition. The set $\Delta_{c}$ is a subsemigroup of $\Delta_{f}$ closed under direct sums, subobjects and conjugates. The family $\left(\rho, \mathscr{U}_{\rho}\right), \rho \in \Delta_{c}$ forms a coherently covariant family of representations (cf. [14; Sect. 8]) in the sense that, for each $\rho, \rho^{\prime} \in \Delta_{c}$,

$$
\begin{aligned}
\left(\rho, \rho^{\prime}\right) & \subset\left(\mathscr{U}_{\rho}, \mathscr{U}_{\rho^{\prime}}\right), \\
\mathscr{U}_{\rho}(a) \mathscr{U}_{\rho \rho^{\prime}}(a)^{-1} & =\rho\left(W_{\rho^{\prime}}(a)\right), \quad a \in \mathbf{R}^{4} .
\end{aligned}
$$

Proof. First we note that (6.11) follows from the uniqueness of $\mathscr{U}_{\rho}$ for a minimal covariant representation. It suffices to consider intertwiners $T \in\left(\rho, \rho^{\prime}\right)$ which are partial isometries with initial and range projections which are minimal in $\rho(\mathfrak{U})^{\prime}$ 
and in $\rho^{\prime}(\mathfrak{Q})^{\prime}$ respectively, since $\left(\rho, \rho^{\prime}\right)$ is the linear span of such elements. Such a $T$ expresses the unitary equivalence of irreducible components of $\rho$ and $\rho^{\prime}$ and $\mathscr{U}_{\rho}, \mathscr{U}_{\rho^{\prime}}$ reduce to the associated minimal representations; hence by uniqueness they are intertwined by $T$.

If $\rho_{1}, \rho_{2} \in \Delta_{c}$ and we define a representation $\mathscr{U}$ by

$$
\mathscr{U}(a)=\rho_{1}\left(W_{\rho_{2}}(a)\right)^{-1} \mathscr{U}_{\rho_{1}}(a), \quad a \in \mathbf{R}^{4},
$$

we have seen that $\left(\rho_{1} \rho_{2}, \mathscr{U}\right)$ is covariant. Moreover it has been shown in [6], in the course of proving Lemma 7.5, that $\mathscr{U}=\mathscr{U}_{\rho_{1} \rho_{2}}$ and hence (6.12) holds whenever $\rho_{1}, \rho_{2}$ are irreducible. From this we shall now deduce that $\mathscr{U}=\mathscr{U}_{\rho_{1} \rho_{2}}$ in general, hence (6.12) will be valid and this will conclude the proof.

Let $T_{i} \in\left(\rho_{i}^{\prime}, \rho_{i}\right)$ with $\rho_{i}^{\prime}$ irreducible, $i=1,2$. Then

$$
T_{1} \times T_{2} \in\left(\rho_{1}^{\prime} \rho_{2}^{\prime}, \rho_{1} \rho_{2}\right) \subset\left(\mathscr{U}_{\rho_{1}^{\prime} \rho_{2}^{\prime}}, \mathscr{U}_{\rho_{1} \rho_{2}}\right) .
$$

To show $\mathscr{U}=\mathscr{U}_{\rho_{1} \rho_{2}}$ it would suffice to show that

$$
T_{1} \times T_{2} \in\left(\mathscr{U}_{\rho_{1}^{\prime} \rho_{2}^{\prime}}, \mathscr{U}\right),
$$

since from (6.13), (6.14) we would get

$$
\mathscr{U}_{\rho_{1} \rho_{2}}(a) T_{1} \times T_{2}=\mathscr{U}(a) T_{1} \times T_{2} .
$$

We could vary $T_{1}, T_{2}$ so that the range projections span the identity and $\mathscr{U}_{\rho_{1} \rho_{2}}=\mathscr{U}$ would follow from (6.15).

To check (6.14) note that (6.12) holds for $\rho_{1}^{\prime}, \rho_{2}^{\prime}$ so that, by the covariance of $\left(\rho_{1}^{\prime}, \mathscr{U}_{\rho_{1}^{\prime}}\right)$

$$
\begin{aligned}
T_{1} \times T_{2} \mathscr{U}_{\rho_{1}^{\prime} \rho_{2}^{\prime}}(a) & =T_{1} \rho_{1}^{\prime}\left(T_{2}\right) \rho_{1}^{\prime}\left(W_{\rho_{2}^{\prime}}(a)^{-1}\right) \mathscr{U}_{\rho_{1}^{\prime}}(a) \\
& =T_{1} \mathscr{U}_{\rho_{1}^{\prime}}(a) \rho_{1}^{\prime}\left(\alpha_{a}^{-1}\left(T_{2} W_{\rho_{2}^{\prime}}(a)^{-1}\right)\right) ;
\end{aligned}
$$

using (6.4) and (6.7) we also have

$$
\alpha_{a}^{-1}\left(T_{2} W_{\rho_{2}^{\prime}}(a)^{-1}\right)=\mathscr{U}_{0}(a)^{-1} T_{2} \mathscr{U}_{\rho_{2}^{\prime}}(a)=\mathscr{U}_{0}(a)^{-1} \mathscr{U}_{\rho_{2}}(a) T_{2}=W_{\rho_{2}}(-a) T_{2},
$$

where we used (6.11) for $T_{2}$. Substituting into the original expression and using (6.11) for $T_{1}$ we get

$$
T_{1} \times T_{2} \mathscr{U}_{\rho_{1}^{\prime} \rho_{2}^{\prime}}(a)=\mathscr{U}_{\rho_{1}}(a) \rho_{1}\left(W_{\rho_{2}}(-a)\right) T_{1} \times T_{2}=\mathscr{U}(a) T_{1} \times T_{2},
$$

where the covariance of $\left(\rho_{1}, \mathscr{U}_{\rho_{1}}\right)$ and the cocycle identities for $W_{\rho_{2}}$ have been used.

We can now state the desired result.

6.3 Theorem. Let $\{\pi, G, \mathfrak{F}\}$ denote the unique normal extended field system with gauge symmetry such that an irreducible representation of $\mathfrak{A}$ fulfilling (1.2) with finite statistics is contained in $\pi$ if and only if it is covariant.

There is a strongly continuous unitary representation $\mathscr{U}$ of the translation group on $\mathscr{H}_{\pi}$ such that $(\pi, \mathscr{U})$ is a minimal covariant representation of $\{\mathfrak{U}, \alpha\}$ and this $\mathscr{U}$ satisfies

(i) $\mathscr{U}(a) \Omega=\Omega, a \in \mathbf{R}^{4}$;

(ii) Spectrum $\mathscr{U} \subset \bar{V}^{+}$;

(iii) $\mathscr{U}(a) \in \pi(\mathfrak{U})^{\prime \prime}, a \in \mathbf{R}^{4}$;

(iv) $\mathscr{U}(a) \mathfrak{F}(\mathscr{C}) \mathscr{U}(a)^{-1}=\mathfrak{F}(\mathscr{C}+a), a \in \mathbf{R}^{4}, \mathscr{C} \in \mathscr{J}$. 
Proof. Obviously, (i), (ii) are just part of the definition of minimal covariant and are just repeated here to stress the symmetry with the statement of Theorem 6.1. The existence of $\mathscr{U}$ follows from the fact that $\pi$ is a direct sum of irreducible components, each covariant and fulfilling the spectrum condition by the remarks above, and from the quoted theorem of [28]. Thus $\mathscr{U}$ is unique.

We need only prove (iv). Actually we will prove directly the existence of $\mathscr{U}$ with properties (i)-(iv) and it will follow that the $\mathscr{U}$ we construct is minimal.

Let $D$ be an arbitrarily chosen double cone at spacelike infinity. Then if $\rho \in \Delta_{c}(D)$ and $\mathscr{U}_{\rho}$ is chosen as above, $\left(\rho^{D}, \mathscr{U}_{\rho}\right)$ is also (minimal) covariant, and by Proposition 6.2 we get a coherently covariant family of representations. We can then apply Theorem 8.4 of [14] to $\pi_{0}^{D}, \mathfrak{A}^{D}$ and to that family of representations.

We obtain in this way a map which assigns to each $\rho \in \Delta_{c}(D)$ a Hilbert space $H_{\rho}$ which, extended to all $\rho \in \Delta_{c}$ as in Eq. (5.15), allows us to define the required field net by

$$
\mathfrak{F}(\mathscr{C})=\left\{H_{\rho}, H_{\rho}^{*} ; \rho \in \Delta_{c}(\mathscr{C})\right\}^{\prime \prime} .
$$

Let $\mathscr{U}$ be the representation of the translation group provided by Theorem 5.4 loc. cit., then $\mathscr{U}(a) \in G^{\prime}=\pi(\mathfrak{U})^{\prime \prime}, a \in \mathbf{R}^{4}$, so that (iii) follows and by Eqs. (8.15), (8.19) of [14] and (5.15) above we have

$$
\mathscr{U}(a) H_{\rho} \mathscr{U}(a)^{-1}=\pi\left(W_{\rho}(a)\right) H_{\rho}=H_{\alpha_{a} \rho \alpha_{a}^{-1}} .
$$

By Eqs. (6.16), (6.17), (iv) follows immediately.

If $\rho \in \Delta_{c}$ is irreducible, we choose $\psi \in H_{\rho}$ with $\psi^{*} \psi=d(\rho) \cdot I$, so that

$$
\left.V \equiv \psi^{*}\right|_{\mathscr{H}_{0}}
$$

is an isometric intertwiner in $\left(\pi_{0} \rho, \pi\right)$ (cf. the proof of Proposition 6.3 of [12]). By (6.17), (6.7), using E) in Proposition 5.9, we have

$$
\begin{aligned}
\mathscr{U}(a) V & =\left.\mathscr{U}(a) \psi^{*}\right|_{\mathscr{H}}=\left.\psi^{*} \pi\left(W_{\rho}(a)^{-1}\right) \mathscr{U}(a)\right|_{\mathscr{H}_{0}} \\
& =\left.\psi^{*}\right|_{\mathscr{H}_{0}} \pi_{0}\left(W_{\rho}(a)^{-1}\right) \mathscr{U}_{0}(a)=V \mathscr{U}_{\rho}(a) .
\end{aligned}
$$

In other words $V$ not only intertwines $\pi_{0} \rho$ and $\pi$ but also $\mathscr{U}_{\rho}$ and $\mathscr{U}$. This proves that, if we identify $\pi$ with the direct sum $\bigoplus_{\xi} d(\xi) \pi_{0} \rho_{\xi}$, then $\mathscr{U}$ is identified with $\bigoplus_{\xi} d(\xi) \mathscr{U}_{\rho_{\xi}}$. Therefore

$$
\mathscr{U}(a) \Omega=\mathscr{U}_{0}(a) \Omega=\Omega,
$$

and $\mathscr{U}$ fulfills the spectrum condition. Properties (i)-(iv) are proved and $(\pi, \mathscr{U})$ is indeed minimal.

We conclude with a remark on Poincaré covariant theories with topological charges. Restricting attention to all $\rho \in \Delta_{f}$ which are covariant for the action $\alpha$ of $\tilde{\mathscr{P}}$ we get a unique corresponding normal extended field system with gauge symmetry. The discussion of this section applies and yields a representation $\mathscr{U}$ of $\widetilde{P}$ fulfilling, as in Theorem 6.1,

(i) $\mathscr{U}(L) \Omega=\Omega, L \in \tilde{\mathscr{P}}$;

(ii) Spectrum $\mathscr{U} \mid \mathbf{R}^{4} \subset \overline{V^{+}}$;

(iii) $\mathscr{U}(\tilde{\mathscr{P}}) \subset \pi(\mathfrak{A})^{\prime \prime}$;

(iv) $\mathscr{U}(L) \mathscr{F}(\mathscr{C}) \mathscr{U}(L)^{-1}=\mathfrak{F}(L \mathscr{C}), L \in \tilde{\mathscr{P}}, \mathscr{C} \in \mathscr{J}$. 
By this result the analysis of [29] can be applied, extending to topological charges the theorem of the connection between spin and Bose or Fermi type of the parastatistics obtained in [9; Theorem 6.4].

It should, however, be possible, if more involved, to derive this result directly without using the existence of a field system. The analogous argument for localizable charges was given in [9].

\section{Concluding Remarks}

In this section we will comment on the situation where the duality assumption is weakened to essential duality. This situation is typical of theories with spontaneously broken gauge symmetries. We will construct both the full gauge group, which might not be compact, in general, and the compact subgroup of unbroken gauge symmetries. We shall limit our discussion to the case of localizable charges.

We will also comment briefly on theories in a spacetime of lower dimensions.

In a theory with spontaneously broken gauge symmetries, the net of local observables no longer fulfills duality [30]. It is, however expected on general grounds [16] to fulfill essential duality. This means that the dual net $\mathfrak{Q}^{d}$ defined by

$$
\mathfrak{I}^{d}(\mathcal{O}) \equiv \mathfrak{U}\left(\mathcal{O}^{\prime}\right)^{\prime}, \quad \mathcal{O} \in \mathscr{K}
$$

is a local net: hence

$$
\mathfrak{A}^{d}\left(\mathcal{O}^{\prime}\right) \subset \mathfrak{A}^{d}(\mathcal{O})^{\prime}=\mathfrak{A}\left(\mathcal{O}^{\prime}\right)^{-} \subset \mathfrak{U}^{d}\left(\mathcal{O}^{\prime}\right)^{-}
$$

and $\mathfrak{I}^{d}$ fulfills duality; moreover

$$
\mathfrak{U}^{d}\left(\mathcal{O}^{\prime}\right)^{-}=\mathfrak{A}\left(\mathcal{O}^{\prime}\right)^{-} .
$$

Thus $\mathfrak{A}=\mathfrak{U}^{d}$ expresses duality whilst $\mathfrak{I}^{d}=\mathfrak{I}^{d d}$ expresses essential duality [31].

A representation $\pi$ of $\mathfrak{A}$ fulfilling (1.1) can be canonically extended to a representation $\pi^{d}$ of $\mathfrak{Q}^{d}$ acting on the same Hilbert space and fulfilling (1.1) and this extension preserves irreducibility and unitary equivalence. Therefore the superselection structure can be analyzed using the net $\mathfrak{A}^{d}[31]$ and the discussion of Sect. 3 applies.

We obtain in this way a complete field system $\{\pi, G, \mathfrak{F}\}$ with gauge symmetry and of course (cf. Sect. 3)

$$
\mathfrak{F} \cap G^{\prime}=\pi\left(\mathfrak{H}^{d}\right) .
$$

By construction, $G$ is a compact group of unitaries leaving the vectors in the vacuum sector elementwise invariant and describing the superselection structure of the theory. It is therefore the unbroken part of the gauge group.

We can now define the full gauge group of the theory to be the group $\mathscr{G}$ of all automorphisms of the $C^{*}$-algebra $\mathfrak{F}$ leaving $\pi(\mathfrak{H})$ pointwise fixed. This terminology is justified since, as will be shown elsewhere, the elements of $\mathscr{G}$ act locally on $\mathfrak{F}$, i.e. they leave each local field algebra $\mathfrak{F}(0)$ globally stable; the subgroup $G$ of $\mathscr{G}$ is the stabilizer in $\mathscr{G}$ of the vacuum state [32].

We can associate another compact group $G_{0}$ to $\mathfrak{A}$ in an intrinsic way. To this 
end, note that representations of $\mathfrak{A}$ satisfying (1.1) with finite statistics are described by localized morphisms $\rho$ of $\mathfrak{U}^{d}, \rho \in \Delta$. Such a $\rho \in \Delta$ will not, in general, leave $\mathfrak{A}$ stable nor will the space of intertwiners $(\rho, \sigma), \rho, \sigma \in \Delta$ be contained in $\mathfrak{A}$. Nevertheless we can consider the transportable localized morphisms of $\mathfrak{A}$ with finite statistics. They correspond to those $\rho \in \Delta$ which leave $\mathfrak{A}$ globally stable and can be localized in any double cone $\mathcal{O}$ by left multiplication with an automorphism induced by a unitary in $\mathfrak{A}$. Let $\mathscr{T}_{0}$ denote the corresponding full subcategory of End $\mathfrak{U}$. It is easily checked that the permutation symmetry $\hat{\varepsilon}$ of $\Delta$ when evaluated on objects of $\mathscr{T}_{0}$ gives arrows in End $\mathscr{U}$ and that $\left(\mathscr{T}_{0}, \hat{\varepsilon}\right)$ is a strict symmetric monoidal $C^{*}$-category with conjugates, subobjects and direct sums. Since we obviously have $(l, l)=\mathbf{C} \cdot I,\left(\mathscr{T}_{0}, \hat{\varepsilon}\right)$ is an abstract dual of a compact group $G_{0}$ [2; Theorem 6.1].

We shall not attempt to discuss the relation of $\mathscr{G}$ to $G_{0}$ in detail. Instead we limit ourselves to pointing out a natural homomorphism of $\mathscr{G}$ into $G_{0}$.

$\mathscr{T}_{0}$ is a subcategory of $\mathscr{T}$ which is embedded in the category $\mathscr{H}(\mathscr{F})$ of Hilbert spaces in $\mathfrak{F}$. Let the embedding functor of $\mathscr{T}_{0}$ in $\mathscr{H}(\mathscr{F})$ be denoted by $H$, then $G_{0}$ is realized as the group of monoidal natural unitary transformations from $H$ to $H$ (cf. [2; Sect. 6]). Since $\pi$ is normal on $\mathfrak{U}^{d}\left(\mathcal{O}^{\prime}\right), \mathcal{O} \in \mathscr{K}$, we have by (7.2) $\pi\left(\mathfrak{U}^{d}\left(\mathcal{O}^{\prime}\right)\right)^{-}=\pi\left(\mathfrak{U}\left(\mathcal{O}^{\prime}\right)\right)^{-}$and hence $\pi(\mathfrak{U})^{-}=\pi\left(\mathfrak{U}^{d}\right)^{-}$. Therefore

$$
\pi(\mathfrak{U})^{\prime} \cap \mathfrak{F}=\pi\left(\mathfrak{U}^{d}\right)^{\prime} \cap \mathfrak{F}=\mathbf{C I},
$$

so that ${ }^{33} \gamma(H(\rho))=H(\rho)$ for each object $\rho$ of $\mathscr{T}_{0}$ and each $\gamma \in \mathscr{G}$. Thus $\gamma$ defines a unitary operator $u_{\rho}$ on $H(\rho)$. The map $\rho \rightarrow u_{\rho}$ is easily checked to be a monoidal natural unitary transformation from $H$ to $H$ and is the image of $\gamma$ under a natural homomorphism of $\mathscr{G}$ into $G_{0}$. This homomorphism will be analyzed more closely elsewhere.

The reader has been warned on various occasions that the analysis presented in this paper applies to local quantum theory without massless particles on the physical Minkowski space. We close this section with brief comments on the cases where our analysis of statistics does not apply.

In the case of topological charges on a spacetime of dimension 3 or lower one meets, as already mentioned, obstacles even in defining the monoidal structure on our categories, which we have not attempted to overcome.

In the case of localizable charges in a 2-dimensional spacetime there is no problem in establishing the monoidal structure on our categories but the very first steps in the analysis of statistics do not, in general, lead to a permutation symmetry making $\mathscr{T}$ into a strict symmetric monoidal $C^{*}$-category. Instead we get a strict braided monoidal $C^{*}$-category where coherence associates to each object unitary representations of the braid groups which will not, in general, factor through the permutation groups $[33]^{34}$.

More precisely, $(\mathscr{T}, \varepsilon)$ will be called a strict braided monoidal $C^{*}$-category if for each pair $\rho, \sigma$ of objects of $\mathscr{T}, \varepsilon(\rho, \sigma) \in(\rho \sigma, \sigma \rho)$ fulfills the assumptions of

33 C.f. the proof of [23; Lemma 2.4].

${ }^{34}$ For an earlier analysis of braid statistics in 2 or 3 dimensional models from a different point of view cf. [34] 
[2, Sect. 2] except (1.6), which is replaced by

$$
\varepsilon(\rho, \sigma \tau)=I_{\sigma} \times \varepsilon(\rho, \tau) \circ \varepsilon(\rho, \sigma) \times I_{\tau} .
$$

This condition would be redundant in the symmetric case; in either case, Eq. (1.7) of [2] is a consequence of the remaining assumptions [35].

The following problem, already posed in [2], arises naturally:

Problem. Can we extend the duality theory for compact groups [2; Theorem 6.1] to more general objects, by replacing strict symmetric monoidal $C^{*}$-categories by strict braided monoidal $C^{*}$-categories? What is the full class of compact group-like objects which arise in this way?

It is not clear to what extent a solution of this problem would aid our understanding of 2-dimensional models. In any case, a simple 2-dimensional model apparently gives rise to a category $\mathscr{T}$ that cannot be embedded monoidally into the category of Hilbert spaces ${ }^{35}$.

Finally, we point out that the analysis of statistics does not cover the case of theories on ordinary Minkowski space involving massless particles. It deserves further investigation to decide whether anomalous statistics can be excluded so that ordinary groups suffice to describe the internal symmetries of the system. We hope to turn to all these problems in future investigations.

Note. We call the reader's attention to the recent paper by $\mathrm{P}$. Deligne [36] of which we learned after submitting this paper and which is devoted to a problem very close to the one considered in [2].

\section{Appendix. Spacelike Cones and Spacelike Infinity}

In order to establish the intertwiner calculus in the case of topological charges we need some simple properties of spacelike cones and spacelike infinity. As the material presented here is of an elementary nature, we have refrained from giving any proofs.

Let $\mathscr{D}:=(n: n \cdot n=-1)$ and we regard $\mathscr{D}$ as representing spacelike infinity. We give $\mathscr{D}$ the causal structure inherited from Minkowski space. If $n, n^{\prime} \in \mathscr{D}$, then $\left(n-n^{\prime}\right) \cdot\left(n-n^{\prime}\right)=-2-2 n \cdot n^{\prime}$ so that the causal structure on $\mathscr{D}$ is determined by the invariant $n \cdot n^{\prime}$. Two distinct points $n, n^{\prime}$ of $\mathscr{D}$ are lightlike if $n \cdot n^{\prime}=-1$ and timelike if $n \cdot n^{\prime}<-1$. In either case we can distinguish positive from negative according to the sign of $n^{\circ}-n^{\prime \circ}$. Finally, $n$ and $n^{\prime}$ are spacelike if $n \cdot n^{\prime}>-1$.

If $n_{+}$and $n_{-}$are points of $\mathscr{D}$ and $n_{+}$is positive timelike to $n_{-}$, then the (open) double cone (of $\mathscr{D}$ ) generated by $n_{+}$and $n_{-}$consists of the set of points of $\mathscr{D}$ which are negative timelike to $n_{+}$and positive timelike to $n_{-}$.

Let $D$ be a double cone in $\mathscr{D}$, then the spacelike cone $\mathscr{C}$ with apex $a$ and base $D$ is the set of points

$$
\mathscr{C}:=\bigcup_{\lambda>0}\{a+\lambda n: n \in D\}
$$

\footnotetext{
${ }^{35}$ We thank K. Fredenhagen and B. Schroer for a discussion on this point
} 
Alternatively,

$$
\mathscr{C}=\left\{a+x: x \cdot x<0, \frac{x}{\sqrt{-x \cdot x}} \in D\right\}=a+\bigcup_{\lambda>0} \lambda \mathcal{O}
$$

where $\mathcal{O}$ is the double cone in Minkowski space with vertices $n_{+}$and $n_{-}$.

The final formula for $\mathscr{C}$ shows that a spacelike cone is a spacelike cone in the sense of Buchholz and Fredenhagen [6]. The converse is not true; in fact, a spacelike cone $\mathscr{S}$ in the sense of [6] is a spacelike cone in the above sense if and only if $\mathscr{S}$ is causally closed, i.e. $\mathscr{S}=\mathscr{S}^{\prime \prime}$.

If $\mathscr{C}$ is a spacelike cone we write $D(\mathscr{C})$ to denote the double cone in $\mathscr{D}$ which is the base of $\mathscr{C}$.

A.1 Lemma. Let $\mathscr{C}$ be a spacelike cone and $x$ a point of Minkowski space then

i) If $n \in D(\mathscr{C})$ then $x+\lambda n \in \mathscr{C}$ for $\lambda \in \mathbf{R}_{+}$sufficiently large.

ii) If $x+\lambda n \in \mathscr{C}$ for $\lambda \in \mathbf{R}_{+}$sufficiently large then $n \in \overline{D(\mathscr{C})}$.

A.2 Corollary. If $\mathscr{C}_{1} \subset \mathscr{C}_{2}$ then $D\left(\mathscr{C}_{1}\right) \subset D\left(\mathscr{C}_{2}\right)$.

A.3 Lemma. Given spacelike cones $\mathscr{C}_{1}, \mathscr{C}_{2}, \ldots, \mathscr{C}_{n}$ and $\mathscr{C}$ with $D\left(\mathscr{C}_{i}\right) \subset D(\mathscr{C})$, $i=1,2, \ldots, n$, there is a spacelike cone $\hat{\mathscr{C}}$ with $D(\hat{\mathscr{C}})=D(\mathscr{C})$ and $\mathscr{C}_{1} \cup \mathscr{C}_{2} \cup \cdots \cup \mathscr{C}_{n} \subset \widehat{\mathscr{C}}$.

A.4 Definition. Let $U$ be a set in Minkowski space, then we say a point $n \in \mathscr{D}$ at spacelike infinity is attached to $U$ if there is a spacelike cone $\mathscr{C}$ with $\mathscr{C} \subset U$ and $n \in D(\mathscr{C})$.

If $\mathscr{C}$ is a spacelike cone then $n \in \mathscr{D}$ is attached to $\mathscr{C}$ if and only if $n \in D(\mathscr{C})$.

A.5 Lemma. If $n \in \mathscr{D}$ is attached to $U_{1}$ and $U_{2}$ then $n$ is attached to $U_{1} \cap U_{2}$.

A.6 Definition. A subset $U$ of Minkowski space is said to be spacelike cone connected if given spacelike cones $\mathscr{C}_{0}, \mathscr{C}_{1} \subset U$ we can define spacelike cones $\mathscr{C}_{2}, \mathscr{C}_{3}, \ldots, \mathscr{C}_{m}$ and $\hat{\mathscr{C}}_{1}, \hat{\mathscr{C}}_{2}, \ldots, \hat{\mathscr{C}}_{m}$ in $U$ with $\mathscr{C}_{i} \cup \mathscr{C}_{i+1} \subset \hat{\mathscr{C}}_{i}$ and $\mathscr{C}_{m} \cup \mathscr{C}_{0} \subset \hat{\mathscr{C}}_{m}$.

The above concept arises naturally in trying to establish an intertwiner calculus for topological charges. The next lemma reduces this concept to a question at spacelike infinity which can be more easily visualized.

A.7 Lemma. A subset $U$ of Minkowski space is spacelike cone connected if and only if the set of points at spacelike infinity attached to $U$ is pathwise connected.

A.8 Lemma. Let $\mathscr{C}$ be a spacelike cone then $n \in \mathscr{D}$ is attached to $\mathscr{C}^{\prime}$ if and only if $n$ is in the interior of the spacelike complement of $D(\mathscr{C})$ in $\mathscr{D}$. This set is pathwise connected.

As a crude first approximation, working with spacelike cones in a spacetime of dimension $d$ is like working with double cones in a spacetime of dimension $d-1$. There are, however, some important differences related to the fact that $\mathscr{D}$ has a different topology from $(d-1)$-dimensional Minkowski space. In particular, the set of double cones in $\mathscr{D}$ is not directed under inclusion and the spacelike complement of a double cone in $\mathscr{D}$ is bounded set.

Here are some words of warning on double cones in $\mathscr{D}$ and hence implicitly about spacelike cones in Minkowski space. The statements refer to the usual 4-dimensional Minkowski space. 
a) Not every double cone $D$ in $\mathscr{D}$ is contained in a double cone based on $x^{0}=0$.

b) There are double cones $D_{1}$ and $D_{2}$ such that $\bar{D}_{1}^{\prime} \cap \bar{D}_{2}^{\prime}$ is not pathwise connected.

c) There are double cones $D_{1}, D_{2}$ and $D_{3}$ in $\mathscr{D}$ such that $\bar{D}_{1}^{\prime} \cap \bar{D}_{2}^{\prime} \cap \bar{D}_{3}^{\prime}$ is the empty set and we may even choosen $D_{1}$ and $D_{2}$ to be based on the same spacelike hyperplane.

Here are the positive results which allow us to develop a good intertwiner calculus in a 4-dimensional Minkowski space.

d) Given two double cones $D_{1}$ and $D_{2}$ in $\mathscr{D}, \bar{D}_{1}^{\prime} \cap \bar{D}_{2}^{\prime}$ is not empty.

e) Given $n, n^{\prime} \in \mathscr{D}$ there are arbitrarily small double cones $D_{1}$ and $D_{2}$ with $n \in D_{1}$ and $n^{\prime} \in D_{2}$ such that $\bar{D}_{1}^{\prime} \cap \bar{D}_{2}^{\prime}$ is pathwise connected.

f) Given $n_{1}, n_{2}, n_{3} \in \mathscr{D}$, there are arbitrarily small double cones $D_{i}, i=1,2,3$ with $n_{i} \in D_{i}$ such that $\overline{D_{1}^{\prime}} \cap \overline{D_{2}^{\prime}} \cap \overline{D_{3}^{\prime}}$ is not empty.

On occasion, we also use

g) Given double cones $D_{1}, D_{2}$ in $\mathscr{D}$, there are double cones $D_{01}, D_{02}, D$ in $\mathscr{D}$ such that $D_{0 i} \subset D_{i} \cap D$.

h) Given two spacelike separated spacelike cones $\mathscr{C}_{1}, \mathscr{C}_{2}$ in $\mathscr{J}$, there are $\mathscr{C}_{01}, \mathscr{C}_{02}, \mathscr{C}$ in $\mathscr{J}$ such that $\mathscr{C}_{0 i} \subset \mathscr{C}_{i} \cap \mathscr{C}$.

Acknowledgements. The final stages of the present collaboration took place while we were both participating in the Symposium on Operator Algebras in 1988-1989 at the Mittag-Leffler Institute in Stockholm, where we found a stimulating atmosphere and perfect working conditions. We would like to thank the Mittag-Leffler Institute for the warm hospitality and support, and Professors Uffe Haagerup and Erling St $\phi$ rmer for having organized the symposium and for the kind invitation.

\section{References}

1. Doplicher, S., Haag, R., Roberts, J. E.: Local observables and particle statistics I. Commun. Math. Phys. 23, 199-230 (1971)

2. Doplicher, S., Roberts, J. E.: A new duality theory for compact groups. Inventiones Math. 98, 157-218 (1989)

3. Wick, G. C., Wightman, A. S., Wigner, E. P.: The intrinsic parity of elementary particles. Phys. Rev. 88, 101-105 (1952)

4. Haag, R., Kastler, D.: An algebraic approach to quantum field theory. J. Math. Phys. 5, 848-861 (1964)

5. Borchers, H. J.: Local rings and the connection of spin with statistics. Commun. Math. Phys. 1, 281-307 (1965)

6. Buchholz, D., Fredenhagen, K.: Locality and the structure of particle states. Commun. Math. Phys. 84, 1-54 (1982)

7. Fredenhagen, K., Marcu, M.: Charged states in $\mathbf{Z}_{2}$ gauge theories. Commun. Math. Phys. 92, 81-119 (1983)

8. Buchholz, D.: The physical state space of quantum electrodynamics. Commun. Math. Phys. 85, 49-71 (1982)

9. Doplicher, S., Haag, R., Roberts, J. E.: Local observables and particle statistics II. Commun. Math. Phys. 35, 49-85 (1974)

10. Doplicher, S., Haag, R., Roberts, J. E.: Fields, observables and gauge transformations I. Commun. Math. Phys. 13, 1-23 (1969)

11. Doplicher, S., Roberts, J. E.: Fields, statistics and non-Abelian gauge groups. Commun. Math. Phys. 28, 331-348 (1972)

12. Doplicher, S., Haag, R., Roberts, J. E.: Field, observables and gauge transformations II. Commun. Math. Phys. 15, 173-200 (1969) 
13. Fröhlich, J.: New super-selection sectors ("soliton-states") in two dimensional Bose quantum field models. Commun. Math. Phys. 47, 269-310 (1976)

14. Doplicher, S., Roberts, J. E.: Endomorphisms of $C^{*}$-algebras, cross products and duality for compact groups. Ann. Math. 130, 75-119 (1989)

15. Cuntz, J.: Simple $C^{*}$-algebras generated by isometries. Commun. Math. Phys. 57, 173-185(1977)

16. Bisognano, J. J., Wichmann, E. H.: On the duality condition for quantum fields. J. Math. Phys. 17, 303-321 (1976)

17. Doplicher, S., Roberts, J. E.: Duals of compact Lie groups realized in the Cuntz algebras and their actions on $C^{*}$-algebras. J. Funct. Anal. 74, 96-120 (1987)

18. Borchers, H. J.: A remark on a theorem of B. Misra. Commun. Math. Phys. 4, 315-323 (1967)

19. Fredenhagen, K.: On the existence of antiparticles. Commun. Math. Phys. 79, 141-151 (1981)

20. Drühl, K., Haag, R., Roberts, J. E.: On Parastatistics. Commun. Math. Phys. 18, $204-226$ (1970)

21. Roberts, J. E.: Statistics and the intertwiner calculus. In: $C^{*}$-Algebras and their applications to statistical mechanics and quantum field theory. Kastler, D. (ed.) pp. 203-225. Amsterdam, New York, Oxford: North Holland 1976

22. Roberts, J. E.: Cross products of von Neumann algebras by group duals. Symposia Mathematica 20, 335-363 (1976)

23. Doplicher, S., Roberts, J. E.: Compact group actions on $C^{*}$-algebras. J. Operator Theory 19, 283-305 (1988)

24. Tannaka, T.: Ưber den Dualitätssatz der nichtkommutativen topologischen Gruppen. Tôhoku Math. J. 45, 1-12 (1939)

25. Roberts, J. E.: Localization in algebraic field theory. Commun. Math. Phys. 85, 87-98 (1982)

26. Doplicher, S.: Local aspects of superselection rules. Commun. Math. Phys. 85, 73-86 (1982); Doplicher, S., Longo, R.: Local aspects of superselection rules II. Commun. Math. Phys. 88, 399-409 (1983); Buchholz, D., Doplicher, S., Longo, R.: On Noether's theorem in quantum field theory. Ann. Phys. 170, 1-17 (1986)

27. Pedersen, G. K.: $C^{*}$-algebras and their automorphism groups. London, New York, San Francisco: Academic Press 1979

28. Borchers, H. J., Buchholz, D.: The energy-momentum spectrum in local field theories with broken Lorentz-symmetry. Commun. Math. Phys. 97, 169-185 (1985)

29. Buchholz, D., Epstein, H.: Spin and statistics of quantum topological charges. Fizika 17, 329-343 (1985)

30. Roberts, J. E.: Spontaneously broken gauge symmetries and superselection rules. Proceedings of the International School of Mathematical Physics, Camerino 1974. Gavallotti, G. (ed.). Università di Camerino 1976

31. Roberts, J. E.: Net cohomology and its applications to field theory. In: Quantum Fields-Algebras, Processes. Streit, L. (ed.). Wien, New York: Springer 1980

32. Buchholz, D., Doplicher, S., Longo, R., Roberts, J. E.: Broken symmetries and degeneracy of the vacuum in quantum field theory, (in preparation)

33. Fredenhagen, K., Rehren, K.-H., Schroer, B.: Superselection sectors with braid group statistics and exchange algebras I. Commun. Math. Phys. 125, 201-226 (1989)

34. Fröhlich, J.: Statistics of fields, the Yang-Baxter equation, and the theory of knots and links. In: Non-Perturbative Quantum Field Theory. 't Hooft, G. et al. (eds.). New York: Plenum Press 1988; Fröhlich, J., Marchetti, P. A.: Quantum field theory of vortices and anyons. Commun. Math. Phys. 121, 177-224 (1989)

35. Joyal, A., Street, R.: Braided monoidal categories. Macquarie Mathematics Studies, 1986

36. Deligne, P.: Categories Tannakiennes. Grothendieck Festschrift (to appear)

Communicated by A. Connes

Received July 12, 1989; in revised form December 18, 1989 
